\title{
Non-Obtrusive Heart Rate \\ and Speed-Distance Monitoring Systems \\ for the Smart Rollator
}

\author{
Submitted by: \\ Emile Richard \\ B. Eng., McGill University \\ A thesis submitted to the Faculty of Graduate Studies and Research \\ in partial fulfillment of the requirements for the degree of: \\ Master of Applied Science \\ Ottawa-Carleton Institute for Biomedical Engineering \\ Department of Systems and Computer Engineering \\ Carleton University \\ Ottawa, Ontario \\ Canada K1S 5B6 \\ January, 2011
}

(C) Copyright Emile Richard, 2011 


$\begin{array}{ll}\begin{array}{l}\text { Library and Archives } \\ \text { Canada }\end{array} & \begin{array}{l}\text { Bibliothèque et } \\ \text { Archives Canada }\end{array} \\ \begin{array}{l}\text { Published Heritage } \\ \text { Branch }\end{array} & \begin{array}{l}\text { Direction du } \\ \text { Patrimoine de l'édition }\end{array} \\ \begin{array}{l}\text { 395 Wellington Street } \\ \text { Ottawa ON K1A ON4 } \\ \text { Canada }\end{array} & \begin{array}{l}395, \text { rue Wellington } \\ \text { Ottawa ON K1A ON4 } \\ \text { Canada }\end{array}\end{array}$

Your file Votre référence ISBN: 978-0-494-79546-0

Our file Notre reférence

ISBN: 978-0-494-79546-0

NOTICE:

The author has granted a nonexclusive license allowing Library and Archives Canada to reproduce, publish, archive, preserve, conserve, communicate to the public by telecommunication or on the Internet, loan, distribute and sell theses worldwide, for commercial or noncommercial purposes, in microform, paper, electronic and/or any other formats.

The author retains copyright ownership and moral rights in this thesis. Neither the thesis nor substantial extracts from it may be printed or otherwise reproduced without the author's permission.
AVIS:

L'auteur a accordé une licence non exclusive permettant à la Bibliothèque et Archives Canada de reproduire, publier, archiver, sauvegarder, conserver, transmettre au public par télécommunication ou par l'Internet, prêter, distribuer et vendre des thèses partout dans le monde, à des fins commerciales ou autres, sur support microforme, papier, électronique et/ou autres formats.

L'auteur conserve la propriété du droit d'auteur et des droits moraux qui protège cette thèse. $\mathrm{Ni}$ la thèse ni des extraits substantiels de celle-ci ne doivent être imprimés ou autrement reproduits sans son autorisation.
In compliance with the Canadian Privacy Act some supporting forms may have been removed from this thesis.

While these forms may be included in the document page count, their removal does not represent any loss of content from the thesis.
Conformément à la loi canadienne sur la protection de la vie privée, quelques formulaires secondaires ont été enlevés de cette thèse.

Bien que ces formulaires aient inclus dans la pagination, il n'y aura aucun contenu manquant. 


\section{Abstract}

The objective of the Smart Rollator project is to enable health care monitoring and quantify rollator usage by embedding electronic sensing and recording. This thesis examines two sensor systems for the Smart Rollator: the electrocardiogram and speed/distance.

A novel ECG amplifier, designed specifically to interface two gel-less electrodes, is introduced. The objective was to develop a circuit with performance sufficient to extract heart rate information reliably using digital signal processing techniques. A prototype system is built and evaluated. Results demonstrate that this circuit may be suitable for the Smart Rollator application.

A previous version of the Smart Rollator included the ability to monitor movement by sensing wheel rotation. A new design, in addition to measuring absolute distance travelled, measures net distance. An empirical design process is described to establish the best mounting distance of the sensors from the plane of the wheel, as well as the best spacing between sensors. 


\section{Acknowledgements}

I extend my sincere gratitude and appreciation to the people who made this master thesis possible.

A special thanks to my supervisor Dr. Adrian Chan for giving me his unrestrained advice and encouragement. In the past two years, he has taught me about the process of research and helped me further develop my knowledge of biosignal acquisition and processing. I am grateful for the time he spent reviewing the material in this thesis.

I also want to thank my family, who supported me throughout this period and made time available for this large undertaking.

Finally, I would like to thank Carleton University's Department of Systems and Computer Engineering for helping provide the financial and research resources needed to complete this work. 


\section{Table of Contents}

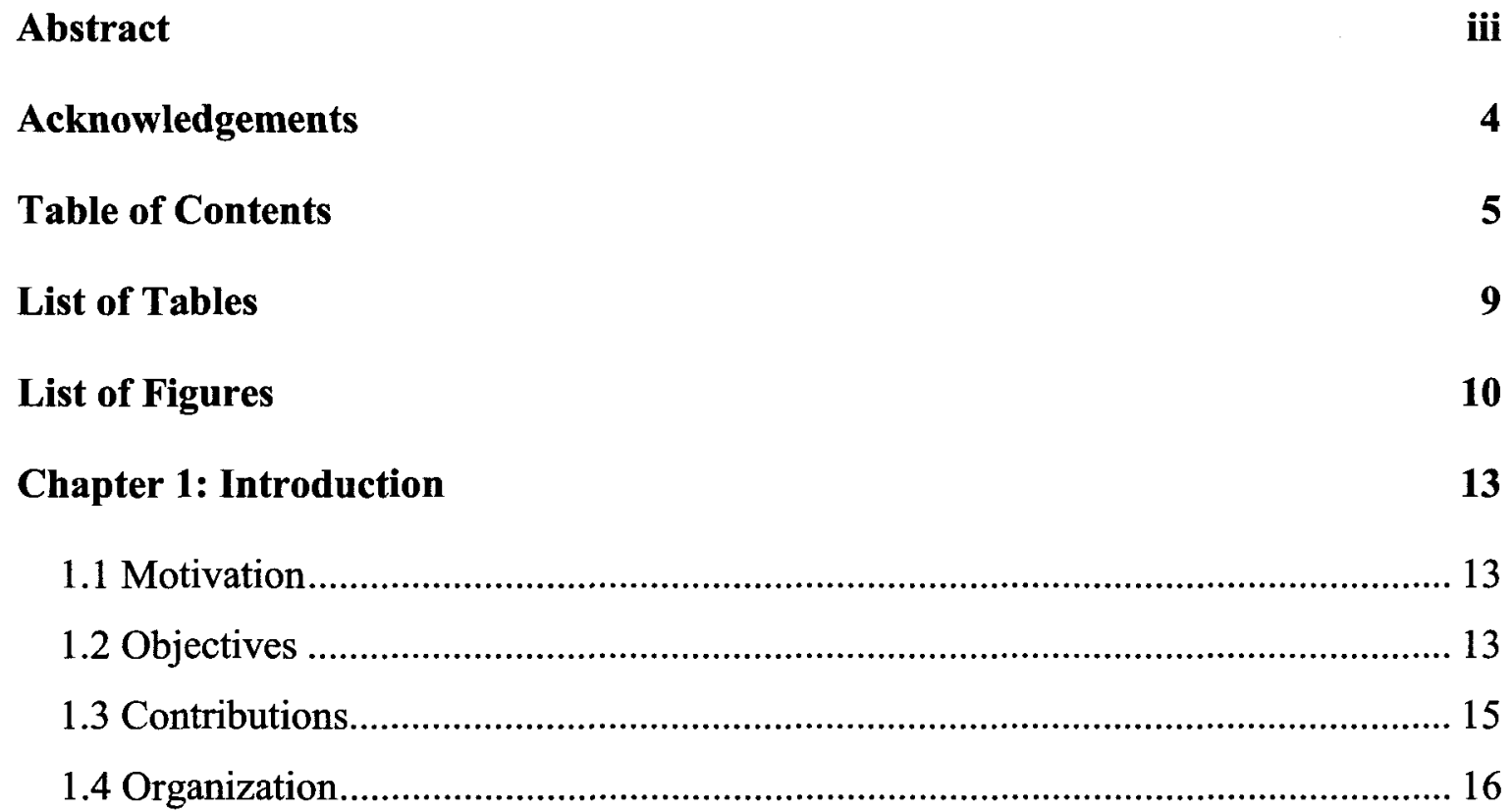

$\begin{array}{ll}\text { Chapter 2: Background } & 18\end{array}$

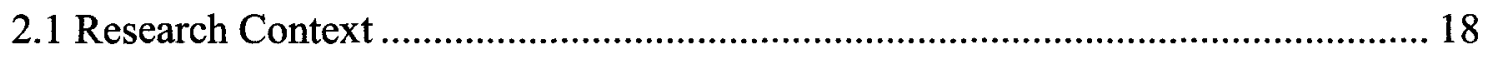

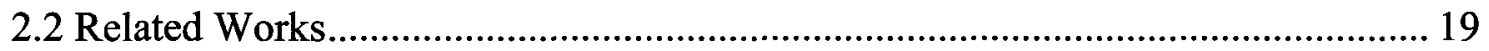

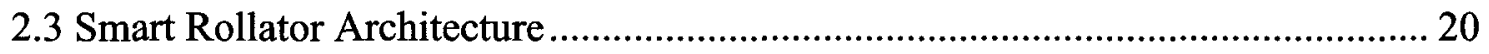

2.4 Smart Rollator Research ......................................................................... 22

$\begin{array}{ll}\text { Chapter 3: ECG Acquisition } & 25\end{array}$

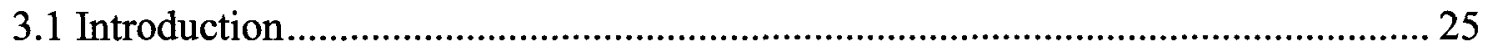

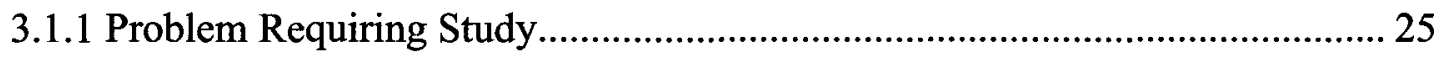

3.1.2 State of Knowledge in ECG Amplifier Design.......................................... 26

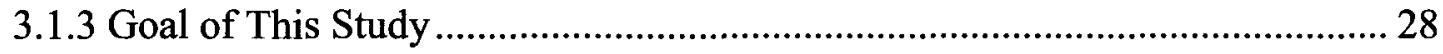

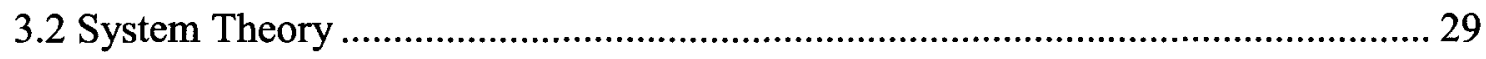

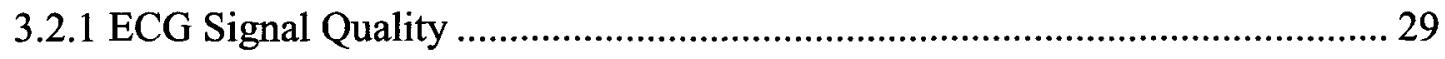

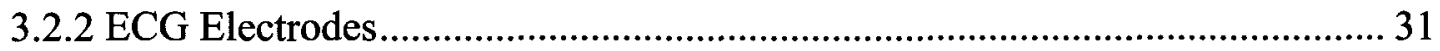




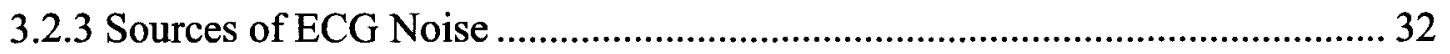

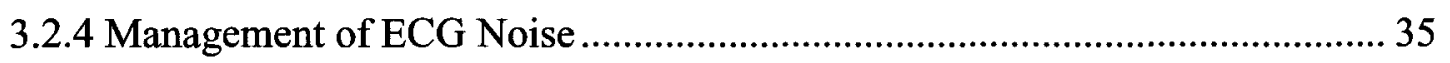

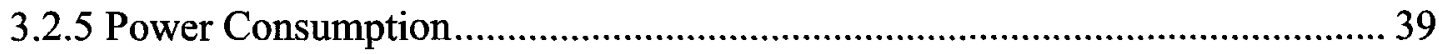

3.2.6 Conventional Three-Electrode ECG Amplifier ............................................... 40

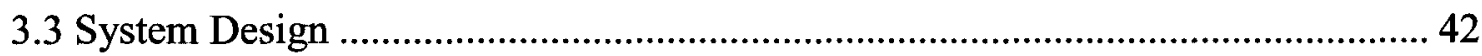

3.3.1 Technical Goals of the Design ....................................................................... 42

3.3.2 Choice of Electrodes ....................................................................................... 43

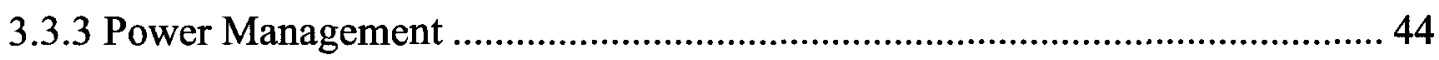

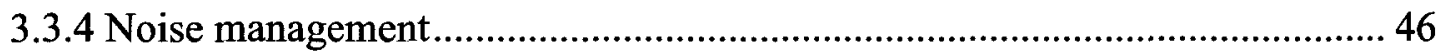

3.3.5 The Proposed Two-Electrode ECG Amplifier................................................. 48

3.3.6 The Reference Three-Electrode ECG Amplifier ............................................. 51

3.3.7 Grip Electrode Description ............................................................................ 53

3.4 Electrode Characterization Tests ...................................................................... 54

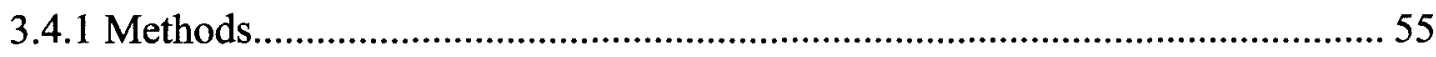

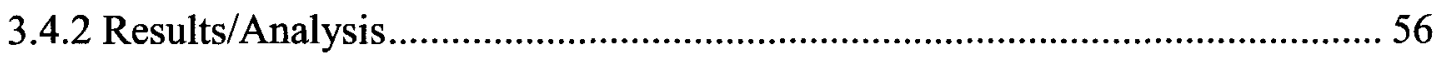

3.5 ECG Amplifier Characterization Tests ................................................................. 59

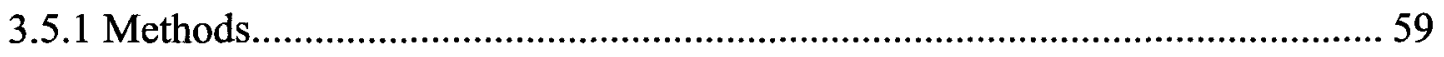

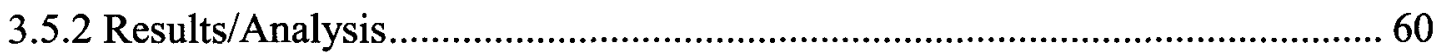

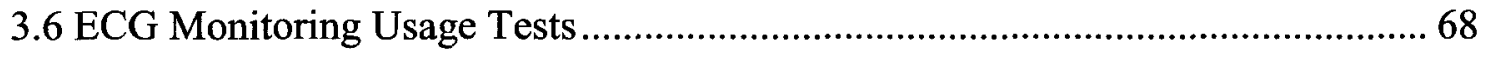

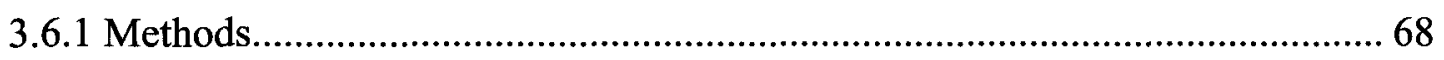

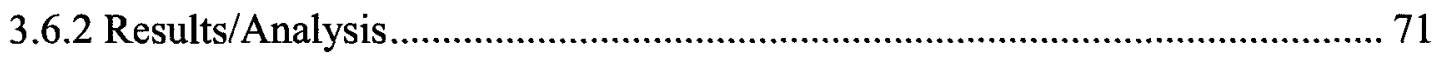

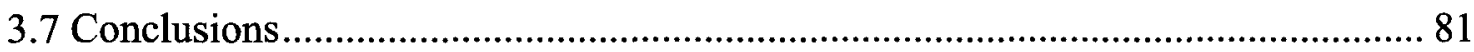

Chapter 4: Speed/Distance Monitoring $\quad 84$

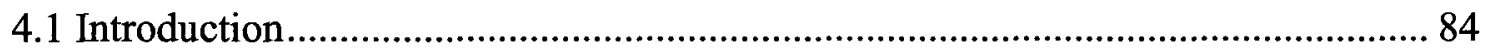

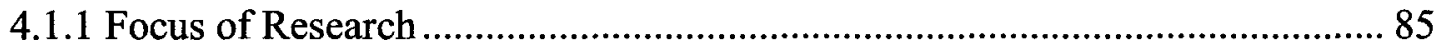

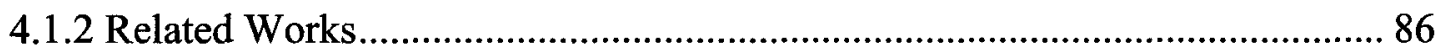

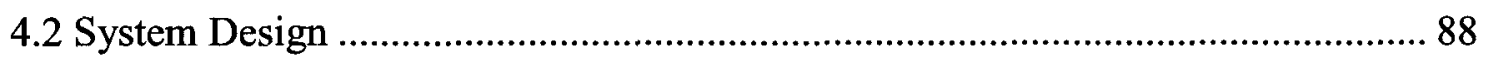

4.2.1 Rotary Quadrature Encoders ........................................................................... 88

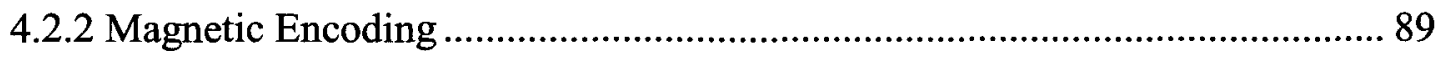

4.2.3 Selection of a Magnetic Sensor.................................................................. 90 
4.2.4 Selection of Magnets.................................................................................... 92

4.2.5 Field Potential and Orientation of Magnets ..................................................... 92

4.2.6 Sensor Distance from Wheel ........................................................................ 98

4.2.7 Sensor Spacing and Phase Quality................................................................ 99

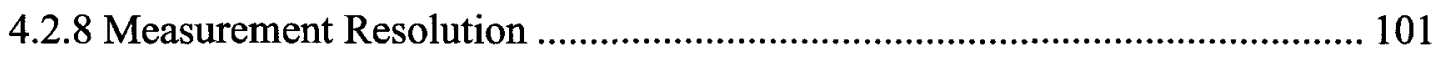

4.2.9 Distance and Speed Calculation..................................................................... 103

4.2.10 Movement Artefact Error............................................................................ 105

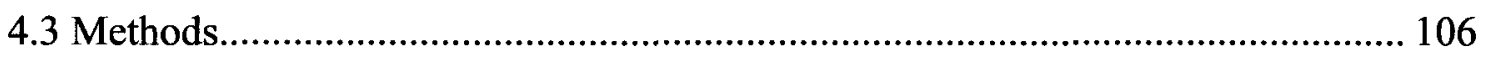

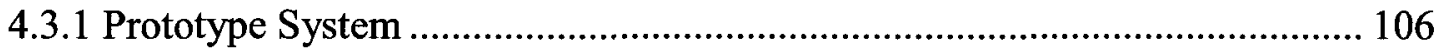

4.3.2 Pulse Quality Measurement ........................................................................ 109

4.3.3 Measurement of Quadrature Signal Validity ................................................. 110

4.3.4 Measurement of Wheel Wobble and Vibration ............................................... 110

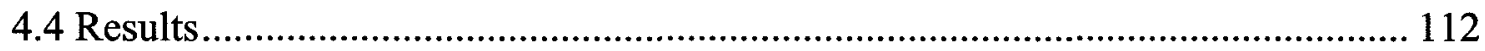

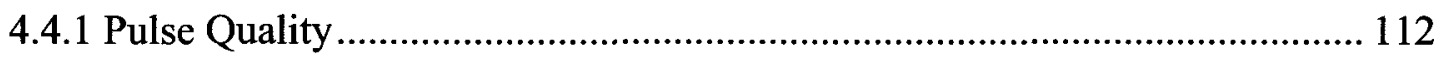

4.4.2 Quadrature Signal Validity .......................................................................... 113

4.4.3 Magnet Positioning Error............................................................................. 115

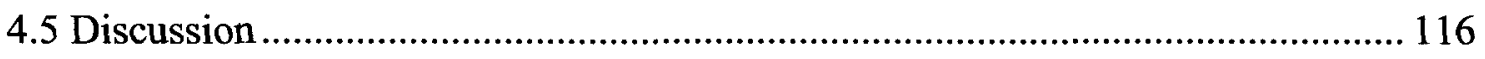

4.5.1 Design of a Simple Pulse Tallying System..................................................... 117

4.5.2 Design of a Quadrature Encoded System ................................................... 117

4.6 Conclusion ...................................................................................................... 120

$\begin{array}{ll}\text { Chapter 5: Conclusion } & 123\end{array}$

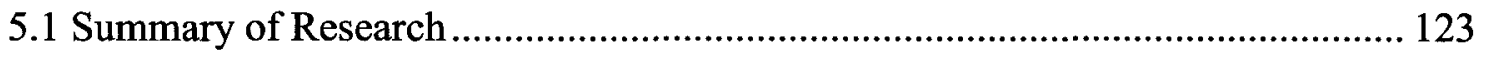

5.1.1 ECG Acquisition ...................................................................................... 123

5.1.2 Speed/Distance Measurement ..................................................................... 125

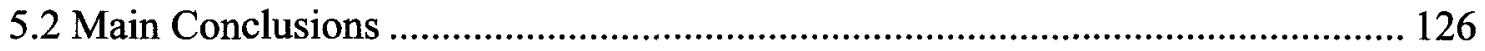

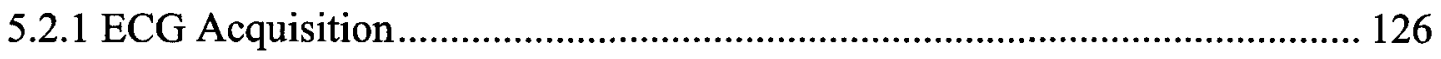

5.2.2 Speed/Distance Monitoring ………............................................................ 127

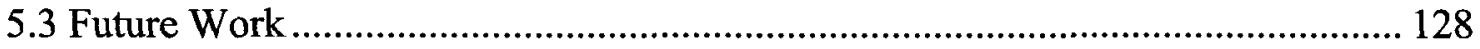

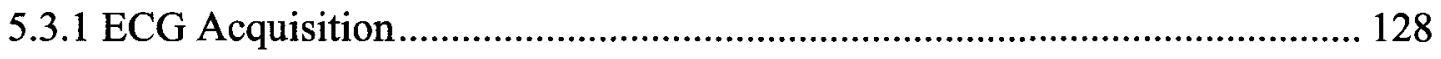

5.3.2 Speed/Distance Monitoring …………........................................................... 129 


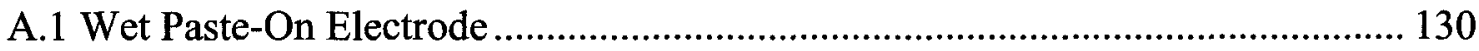

A.2 Instrumentation Amplifier......................................................................... 132

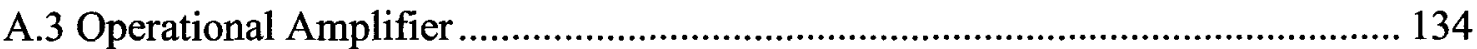

A.4 Lithium Coin Battery ………………................................................................ 136

Appendix B: Parts Specifications - Speed/Distance 138

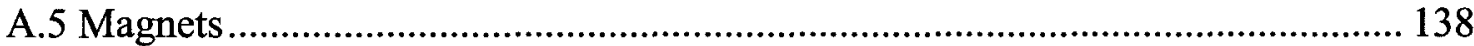

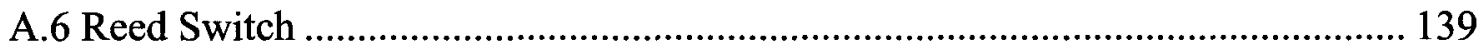

$\begin{array}{lr}\text { References } & 141\end{array}$ 


\section{List of Tables}

Table 2-1: Architecture of the Smart Rollator monitoring system.

21

Table 3-1: Average power consumption of proposed and reference ECG amplifier

circuits.

Table 3-2: Filter details for attenuation of EMG noise (low-pass) and motion artefact (high-pass) 71

Table 4-1: Advantages and limitations of some popular magnetic sensors. 91

Table 4-2: Range of phase lags for valid quadrature signal. 100

Table 4-3: For quadrature decoding of A and B signals, a unit increment or decrement results from each sensed signal transition. The level of the alternate channel is used to establish if the edge represents a clockwise $(+1)$ or counter clockwise $(-1)$ movement. 104 Table 4-4: Specifications of the prototype and rollator systems...................................... 108

Table 4-5: Measured wheel play, wheel wobble and magnet misalignment. 115

Table 4-6: Design parameters and specifications for a robust magnetic quadrature encoder. 


\section{List of Figures}

Figure 1-1: The Smart Rollator system....................................................................... 14

Figure 2-1: The Smart Rollator system combines minimally-obtrusive sensing embedded in a rollator with wireless data uploads to a server for remote storage and analysis........ 20 Figure 2-2: A proposed core system for the Smart Rollator.............................................. 23 Figure 3-1: The ECG wave for a healthy individual. (Horizontal axis is not to scale.) ... 29 Figure 3-2: Dry electrode ECG amplifier with optional driven reference electrode ........ 41 Figure 3-3: Proposed two-electrode ECG amplifier

Figure 3-4: Schematic of a conventional ECG amplifier with driven reference electrode52 Figure 3-5: Rollator handle equipped with the prototype dry electrode and "thumb" electrode. 53

Figure 3-6: Original prototype of ECG circuit with grip-style dry electrodes. 54

Figure 3-7: Skin-to-electrode interface impedance for popular wet $\mathrm{Ag}-\mathrm{AgCl}$ electrodes (circles), and large dry grip-style tin electrodes (triangles). Impedance magnitude is plotted on the left, phase lag on the right.

Figure 3-8: Input impedance of the proposed ECG amplifier (left) and reference circuit (right).

Figure 3-9: Power-line noise model for two-electrode ECG amplifier. 64

Figure 3-10: Frequency response (top plots) and spectral common-mode rejection ratio (bottom plots), for proposed ECG amplifier (left) and for three-electrode reference circuit (right).

Figure 3-11: Representative samples of ECG for subject \#1 and \#2, while standing still. Top plots for each subject are for the proposed two-electrode ECG amplifier. The bottom plots are for the standard three-electrode amplifier. 72

Figure 3-12: Representative samples of ECG for subject \#3 and \#4, while standing still. Top plots for each subject are for the proposed two-electrode ECG amplifier. The bottom plots are for the standard three-electrode amplifier. 73 
Figure 3-13: Representative samples of ECG for subject \#1 and \#2, during a slow walk. Top plots for each subject are for the proposed two-electrode ECG amplifier. The bottom plots are for the standard three-electrode amplifier. 75

Figure 3-14: Representative samples of ECG for subject \#3 and \#4, during a slow walk. Top plots for each subject are for the proposed two-electrode ECG amplifier. The bottom plots are for the standard three-electrode amplifier. 76

Figure 3-15: Representative samples of ECG for subject \#1 and \#2, while gripping tightly on the electrodes. Top plots for each subject are for the proposed two-electrode ECG amplifier. The bottom plots are for the standard three-electrode amplifier. 78 Figure 3-16: 60-second ECG recordings for one subject, including a period of (a) standing still, (b) walking slowly and (c) griping the electrodes tightly. 80 Figure 4-1: Example of a commercially available rollator (left) and the Smart Rollator monitoring system being developed at Carleton University (right). 84

Figure 4-2: First generation distance/speed sensing on the SmartRollator system. 85 Figure 4-3: Rotary encoders (a) measure rotation by sensing edges and levels of two outof-phase slotted tracks (b). Angular displacement is measured by counting transitions, with the direction of each sensed displacement being indicated by the polarity of the other channel (c) 88

Figure 4-4; Spatial distribution of magnetic potential for cases in which magnets are arranged in with alternating polarity (top) and unidirectional polarity (bottom). The solid line indicates the plane of the wheel. Dashed lines show possible trajectories of the magnetic sensors. The y-axis in this plot represents distance from the plane of the wheel. The $\mathrm{x}$-axis represents radial distance (inter-magnet separation along the circumference of the track of magnets.). 94 Figure 4-5: Magnetic potential along a line above the track of magnets, as a function of position. The left plot shows bipolar swings when using the alternating configuration. The right plot, for the unidirectional magnet configuration, shows an always-positive magnetic potential and less intense fluctuation. 
Figure 4-6: Response of a magnetic sensor mounted above a rotating track of magnets (a). The width of the generated pulses (b) is a function of the distance the sensor is mounted from the plane of the wheel. 98

Figure 4-7: Two reed sensors with a small separation distance mounted above the track of rotating magnets (a) will generate a pair of slightly out of phase pulse trains (b). The phase difference will depend on the separation distance.

Figure 4-8: Test system consisting of a rotating disk with countersunk magnets (a) and a magnetic sensor mounted on a fixed bracket (b). To assess the effect of separation distance, a second magnetic sensor was mounted next to the first one (c). 107

Figure 4-9: Three reed switches are used in testing the system. S1 and S2 are above the track of magnets, generating the quadrature A and B signals. S3 generates one pulse per rotation, to help isolate and characterize individual magnets on the wheel. 108 Figure 4-10: On versus off time (duty cycle) of magnetic sensor for rotating wheel with ten magnets. Plotted as a function of distance mounted from wheel and size of magnets used.

Figure 4-11: Effect of separation distance between two sensors on the quality of the quadrature signal. Phase quality is plotted as the ratio of A-to-B phase lag time to " $\mathrm{A}$ " pulse-asserted time, as a function of sensor separation, sensor mounting distance from the wheel, and magnet size. Note that the signal is only usable when the phase lag ratio is between $0 \%$ and $100 \%$.

Figure 4-12: The quadrature signal measured with the wheel rotating at a constant speed. The duty cycle and phase difference were set by adjusting the sensor-to-wheel distance and spacing of the sensors, respectively. 119

Figure 5-1: Datasheet for the A10043 Ag-AgCl paste-on electrode. 131

Figure 5-2: Datasheet for AD623 instrumentation amplifier. 133

Figure 5-3: Datasheet for Texas Instruments TLV2404 operational amplifier. 135

Figure 5-4: Datasheet for Renata CR2032 lithium coin battery.

Figure 5-5: Hamlin 59065 reed switch datasheet. 140 


\section{Chapter 1: Introduction}

\subsection{Motivation}

The over 65 age demographic is a growing population segment, expecting to double by the year 2036 (Turcotte \& Schellenberg, 2007). Challenges associated with aging include increased health problems and decreased mobility. Rollators and other ambulatory assistive devices are often used to overcome mobility and stability problems, improving the ability of individuals to live independently.

Research which advances independent living and promotes health is an international priority. Inactivity, particularly among the elderly, has been shown to be a significant cause of increased morbidity and premature mortality (Harper \& Lyles, 1988) (Hirvensalo, Rantanen, \& Heikkinen, 2000).

Developing solutions to address these needs will benefit not only the older population but all people with disease or injuries impacting their mobility.

\subsection{Objectives}

The objective of the Smart Rollator project is to integrate non-obtrusive biomedical instrumentation and remote monitoring technologies for:

$>$ improved health care through monitoring of adherence and progression during rehabilitation, as well as potential early identification of health care issues; and 
quantitative assessment of rollator usage to improve the design and prescription of rollators. (Chan \& Green, 2008)

The project retrofits a commercially available rollator to enable reliable quantitative measurement during daily use. Data acquired are transferred automatically to a data server, via local data terminal (e.g., located near the user's bed for daily data transfers) and can be accessed by variety of people for analysis (Figure 1-1). The work presented in this thesis focuses on two sensor systems for the Smart Rollator: the electrocardiogram (ECG) and speed/distance.

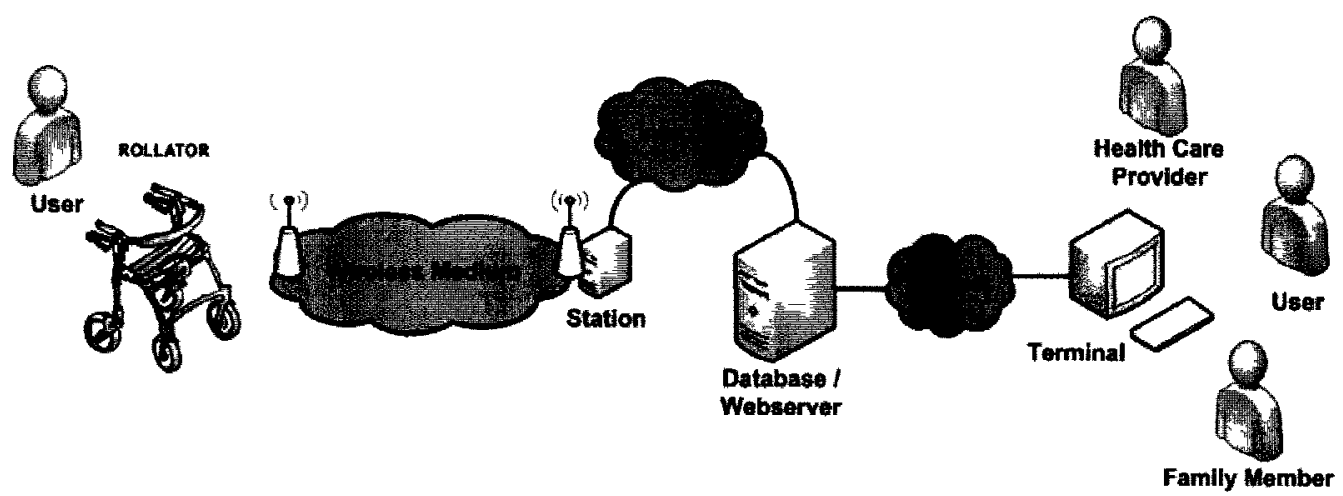

Figure 1-1: The Smart Rollator system

The objectives of this thesis are:

1. to research and design an ECG circuit for the Smart Rollator, capable of acquiring ECG suitable to derive heart rate information; and

2. to research and design a robust speed/distance sensor system.

The ECG design targets a two gel-less electrode interface, appropriately addressing issues such as power, motion artefact, and noise/interference. The proposed speed/distance sensor system enables monitoring of direction (forward/backward) and resolves errors associated with rocking motion. The effects of magnet characteristics and 
placement are examined. While the target application of this work is the Smart Rollator, the outcomes of this research are applicable to other mobility assistive devices (Lankenau \& Röfer, 2000) and remote monitoring applications (Korhonen, Parkka, \& Van Gils, 2003).

\subsection{Contributions}

This thesis focuses on advancing two of the subsystems of the Smart Rollator platform: ECG acquisition, with the goal of measuring heart rate during rollator use, and speed/distance measurement, to help in assessing the amount of and character of motionrelated rollator activity.

The contributions we present in this document are:

\section{Research, design and evaluation of a two-electrode ECG amplifier.}

The particular requirements for an ECG amplifier for the Smart Rollator are identified and a design to satisfy these is researched, designed, built and tested. A conventional three-electrode ECG amplifier is also built and tested (on the same platform), as a point of comparison. Bench top and pilot user data are presented and discussed. Parts of this work were disseminated in a MeMeA conference article (Richard \& Chan, 2010).

\section{Characterization of dry metal grip electrodes for ECG acquisition.}

The performance of simple gel-less plate metal grip electrodes were compared to that of popular clinical-grade wet ECG electrodes. The bioimpedance of their skin-to-electrode interfaces are measured and the implications to ECG acquisition 
discussed. ECG monitoring using these electrodes on four test subjects is performed (as part of the previous contribution).

$>$ Empirical design process for an improved speed/distance measurement system.

The characterization of a measurement system using magnets and magnetic sensors, to measure the distance travelled by a wheeled device, is performed. The result is the ability to determine if and how a robust system can be configured using a given choice of system components. By adding an extra sensor, direction of wheel travel is sensed through quadrature encoding, with additional design advantages. Such a system is characterized, configured, tested and discussed.

\subsection{Organization}

In addition to the current introductory chapter, this thesis consists of four other chapters.

Chapter 2 presents an overview of the Smart Rollator project: its motivation, objectives, as well as our contributions towards the effort.

Chapter 3 discusses a design for non-obtrusively measuring heart rate using ECG monitoring using grip-style dry electrodes. The proposed two-electrode ECG design is compared to a conventional three-electrode ECG design. The creation and bench top characterization of a prototype portable ECG acquisition system are detailed. Preliminary user tests are performed to evaluate its performance, at rest and during moderate activity.

Chapter 4 proposes an improvement to the present system for measuring of speed and distance for a wheeled assist device. A detailed empirical method is proposed for choosing components and configuring the system for robust performance. 
Finally, Chapter 5 concludes this document, summarizing the work performed, the key results and the conclusions from experiments. We finish with a discussion of its impact on the Smart Rollator project and future directions of research. 


\section{Chapter 2: Background}

\subsection{Research Context}

In the next 25 years the number of older adults in Canada is expected to increase to 10 million, with nearly 1 in 4 Canadians having reached the age of 65 (Turcotte \& Schellenberg, 2007). Similar trends are evident worldwide. The ability to maintain mobility and independence is an important factor in a person's health and well-being, as well as in their quality of life (Turcotte \& Schellenberg, 2007). With one third of the community dwelling elderly people and half of nursing home residents having at least one fall annually (Fuller, 2000), ambulatory assistive device research is clearly warranted. In Canada, falls account for $85 \%$ of injuries in the elderly and costs approximately $\$ 2.8$ billion a year (B.C. Ministry of Health Planning, 2004).

There exists a growing senior population that requires increased health care but prefers to live independently. This provides the impetus for remote monitoring technologies that promote independent living and effective health care, including prevention and early detection of health care issues. Advances in sensors, processing and wireless communications have enabled feasible, cost-effective technological solutions. Sensors are smaller and cheaper. Widespread use of personal electronics (e.g. cell phones) has led to the development of processors with more computational power as well as decreased power requirements and cost. Wireless communication is expanding rapidly and has led to the introduction of faster and cheaper data communications using lower- 
power wireless transceivers. There is a high demand for remote monitoring technologies designed for the independent living of older adults. Ambulatory assistive devices, such as rollators, provide an effective means of delivering such solutions.

\subsection{Related Works}

There are other research programs related to the Smart Rollator project. The vast majority are focused on obstacle detection and avoidance, as well as the integration of guidance and wayfinding systems. These include the Utah State University iWalker (Kutiyanawala, Kulyukin, \& LoPresti, 2006), the M.I.T. Personal Aid for Mobility and Monitoring system (Yu, Spenko, \& Dubowsky, 2003), the University of Virginia assistive robotic agent (Wasson, Sheth, Alwan, Granata, Ledoux, \& Huang, 2004) and the Veterans Affairs Personal Adaptive Mobility Aid (Rentschler, Simpson, Cooper, \& Boninger, 2008). The Toronto Rehabilitation Institute's iWalker project (Tung, et al., 2007) (not to be confused with the project of the same name at Utah State University) has research objectives that are closer to that of the Smart Rollator research program. Both are related to reliable monitoring in real-world situations. The Toronto iWalker project's overall research objective is to better understand behaviours and environments that challenge safe mobility of older adult users.

Reliable monitoring during normal use can provide important clinically relevant information. For example, for patients with chronic obstructive pulmonary disease, rollator use can increase walking distance (Gupta, Goldstein, \& Brooks, 2006) and efficiency (Probst, et al., 2004). However, translating such findings with real-world rollator use can be difficult in a complex, dynamic environment (noise, movement, etc.). 


\subsection{Smart Rollator Architecture}

The Smart Rollator is comprised of multiple minimally-obtrusive monitoring subsystems integrated into a commercially available rollator (Figure 2-1, left). Technology is being integrated in a manner such that it remains unnoticed by the user during normal daily activities. The planned sensory subsystems include functions such as distance/speed monitoring, force monitoring, and cardiac monitoring (see Table 2-1 for a complete list of subsystems). Core subsystems provide common services to the sensing and communication subsystems. Each subsystem includes hardware and control software.

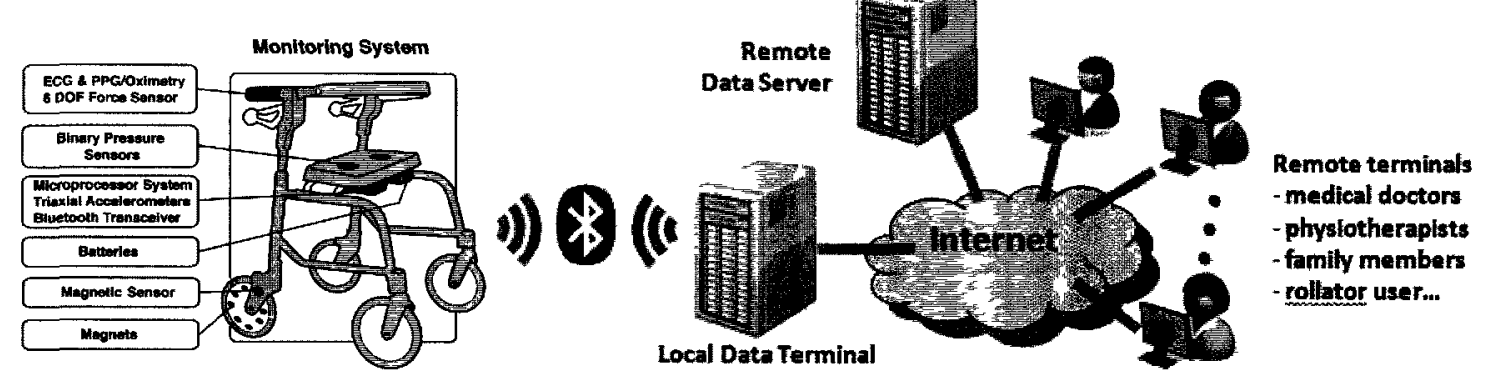

Figure 2-1: The Smart Rollator system combines minimally-obtrusive sensing embedded in a rollator with wireless data uploads to a server for remote storage and analysis.

An embedded microprocessor collects data into local memory, which is then periodically transferred to a remote data server via a local data terminal. This local data terminal may be situated by the user's bed, enabling data transfers each night. Automatic wireless transmission from the rollator to the local data terminal is used to avoid unnecessary user interfaces that may be confusing for the target older adult population. 
Table 2-1: Architecture of the Smart Rollator monitoring system.

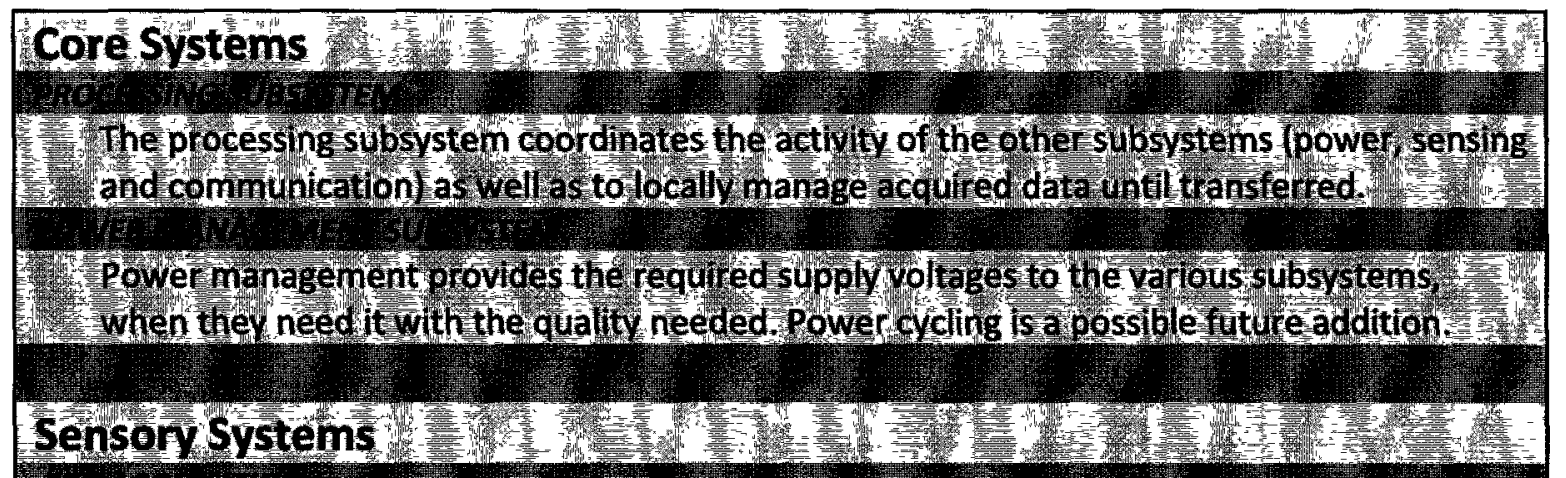

Electrocardiographic data can be acquired through electrodes embedded in the handle grips. Signal amplification and processing issues related to heart rate measurement during user activity are being identified and addressed in the design.

A LED/photodiode sensing system is used to measure the blood fow pulse fas an alternates? method of heart beat mesurement, as well as to calculate blood oxygen content.

The handles of the rollator are equipped with ix degrees of freedom (DOF) pressure sensors. These are able to monitor balance and leaning force vectors.

Digial pressure sensors monitor use of the rollator ' brakes and seat This data helps assess the context of device use events ( $\mathrm{e}$, $\mathrm{g}$, balance issues) and physiological state

Environmental temperature and pressure data can be valuable in better understanding patterns of use with respect to external conditions, providing context t t may be combined for example, with physiological readings (such as heart rate and speed of travel) to assess: body response to stress factors. =

Continuously monitors distance and speed of rollator travel. Wheel otation is measured Wsing maghetic sensors and magnets embedded into the wheel(s). W W

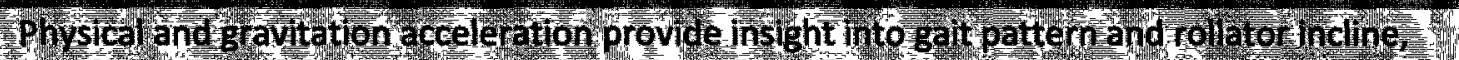
respectively. Also, sudder acceleration mady indicate falls (or hear falls), valuable feedback for design mporovement by the nanufacturer and for updating of usage guidelines.

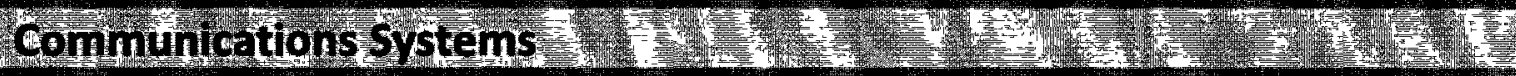

A Bluetooth transcelver provides regular uploads of acquired data to a database sto re as

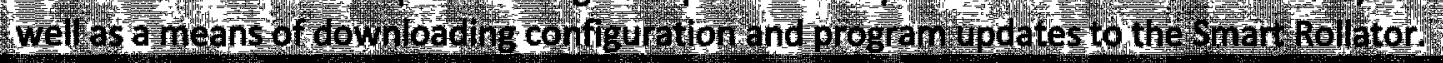

This is a service port alowing the smart Rollat to to be reconfigured or programmed

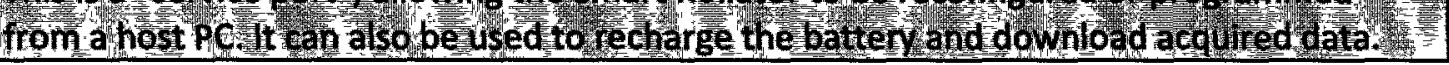


Remote data terminals enable a variety of people (e.g., physiotherapists, family) to access and examine the data, and generate reports (Figure 2-1, right). Automated alarms or notifications regarding potential health problems can be issued appropriately. For example, changes in rollator loading forces may be indicative of a neuromuscular disorder or injury (e.g., asymmetric or increased loading) (Romero \& Stelmach, 2003). A health care professional can be notified to examine such changes and as well as long-term trends, and follow up during the user's subsequent visits. Serious trauma (e.g., falls) would require more immediate attention (e.g., 911).

\subsection{Smart Rollator Research}

In this project, monitoring subsystems are being implemented independently of one another but having a common platform for their eventual combination. Off-the-shelf sensors are being primarily used for research and development of the prototypes and being embedded into rollators.

An initial prototype was developed, which included a speed/distance sensor system that used a magnetic reed switch sensor. Data was being collected using a microcontroller system (Motorola 68HC11) and interfaced to a wireless Bluetooth transceiver capable of performing data transfers to an open source database (MySQL, Sun Microsystems). Remote database queries could be made to generate usage data reports and graphs. The other sensing modalities underwent preliminary evaluation (e.g., bench-top testing), to characterize the sensors and ensure that they meet the technical requirements for the application. 
The research effort is iterative in that it identifies opportunities for improvement in the core, sensor and communication subsystem designs, exploring possible solutions using modeling, simulation, prototyping, and testing techniques. For instance, an improvement to the processing subsystem, to greatly increase the processing power, local data storage and sensor support, while maintaining low power and size, is being proposed (Figure 2-2). It includes a low-power 32-bit ARM based microcontroller with 1MB of data RAM and $0.5 \mathrm{MB}$ of program space, greatly increasing data handling capabilities. The large number of peripheral functions integrated into the STM32 will simplify the design and improve the performance of the various sensor subsystems. Such an updated core subsystem would interface to the already developed sensor subsystems.

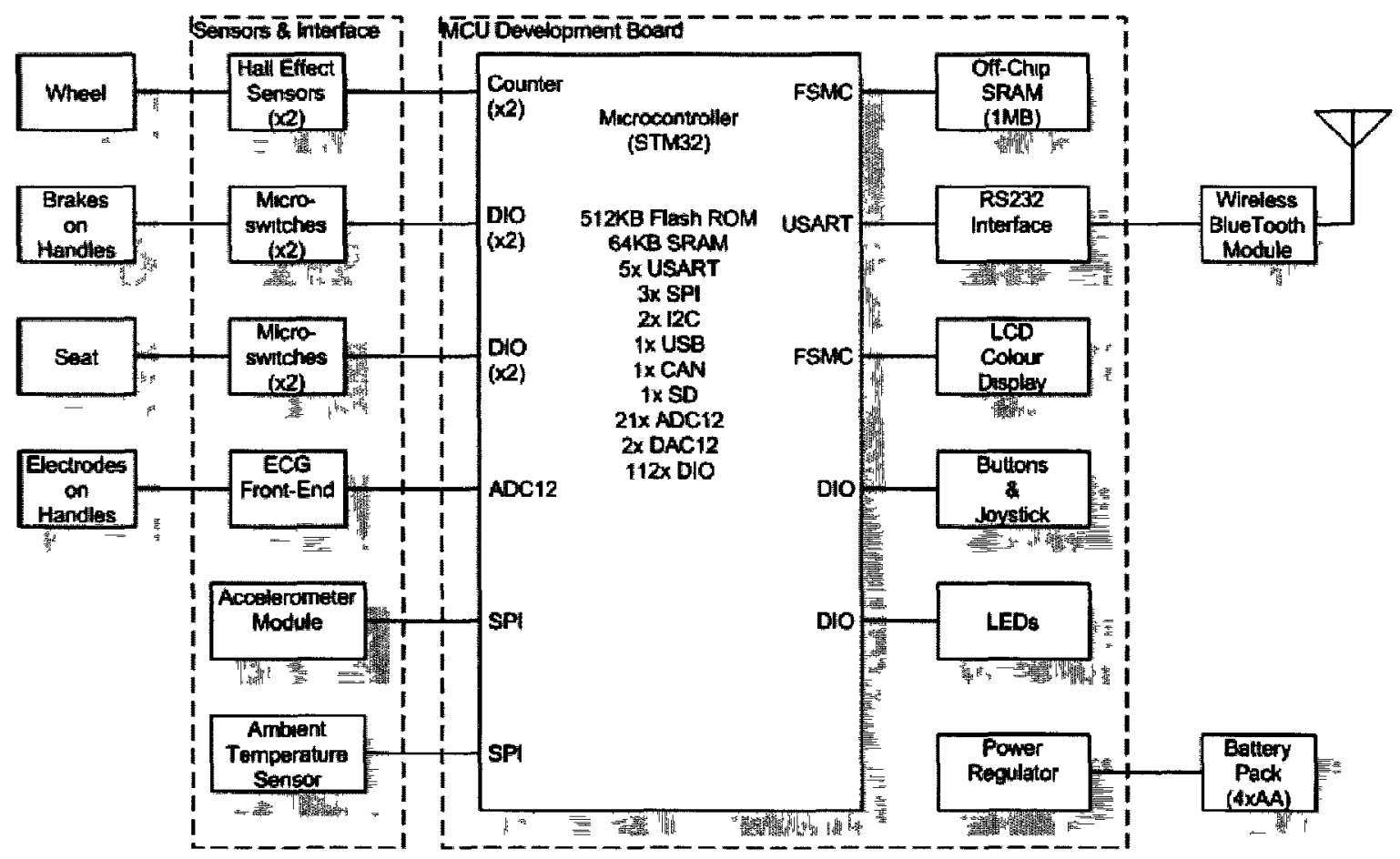

Figure 2-2: A proposed core system for the Smart Rollator. 
This thesis advances the Smart Rollator research effort by offering new designs for two of the above sensor subsystems: ECG acquisition and speed/distance monitoring. Proposed solutions are prototyped and tested. Discussion of results and recommendations for future work are provided. 


\section{Chapter 3: ECG Acquisition}

\subsection{Introduction}

Valuable information about a person's physiological state and health may be ascertained by one's heart rate (HR) and its derivative, heart rate variability (HRV) (Kannel, Kannel, Paffenbarger Jr, \& Cupples, 1987) (Gillum, Makuc, \& Feldman, 1991). As the electrocardiogram (ECG) is relatively easy to acquire and HR simple to determine from the ECG (Ruha, Sallinen, \& Nissila, 1997), ECG acquisition is a good candidate for embedding into commonly used health appliances for the aging population. To this end, heart rate monitoring is presently being included in the design of the Smart Rollator (Chan \& Green, 2008).

A heart rate monitor typically begins with acquisition of the ECG signal through a set of skin electrodes. A specialized ECG amplifier is used to isolate the signal from most of the noise. Further processing, performed either in hardware or software, provides additional filtering, identifies the heart beats, and estimates the HR (reciprocal of the beat-to-beat interval time).

\subsubsection{Problem Requiring Study}

A major challenge when acquiring an ECG signal is mitigating the effects of motion. Contraction of muscles in the vicinity of the ECG electrodes will result in interference from electromyography (EMG) signals. In addition, movement of the electrodes against 
the subject can cause large DC offset changes in the skin-to-electrode interface resulting in significant baseline wander (Webster, 2009). Unmanaged, these DC offsets may easily overwhelm the ECG signal and even saturate the ECG amplifier, especially in low supply voltage designs. Appropriate filtering and signal clamping prior to amplification can help in reducing the number of and length of resulting saturation events.

Power-line interference is another significant component of noise in the ECG signal. This results from capacitive coupling to the body, electrodes, leads and circuit, as well as magnetic coupling to the loop created by the electrode leads (Huhta \& Webster, 1973) (Chimene \& Pallas-Areny, 2000). The 60-Hz common-mode (CM) signal from the body is typically the largest (though not the only) of these noise components. Its effects can be greatly mitigated by (a) using a differential amplifier with high input impedance and common-mode rejection ratio (CMRR), (b) reducing the source impedance of the input (e.g. reducing the skin-to-electrode interface impedance), and (c) effectively decoupling the circuit ground from earth ground at power-line frequency. Since the power-line interference is fixed-frequency, so long as the signal does not saturate or get significantly distorted by the amplifier, a high quality notch filter can also be an effective method to reduce its effects.

\subsubsection{State of Knowledge in ECG Amplifier Design}

For the Smart Rollator application, a two-electrode ECG acquisition system is proposed with dry electrodes embedded in the rollator handles. Most ECG systems have a third electrode as a ground, including many exercise bikes. For the Smart Rollator, a two- 
electrode system is preferred because unlike a bike, we cannot guarantee the user will comply with a certain grip.

Numerous designs for two-electrode ECG amplifier have been proposed (Thakor \& Webster, 1980) (Winter \& Webster, 1983) (Pallas-Areny, 1986), with much discussion and analysis on eliminating CM noise without having to drive the body with a reference signal. Designs which include an active front-end, in which current sources at the amplifier inputs create distinct DM and CM input impedances, have also been presented as a solution (Dobrev \& Daskalov, 2002) (Dobrev D. , 2004) (Dobrev, Neycheva, \& Mudrov, 2008), though at the expense of some added circuit complexity.

Some ECG amplifiers, designed specifically for dry electrodes, provide high fidelity ECG output, meeting American Heart Association requirements for electrocardiographic equipment (Burke \& Assambo, 2007) (Bailey, et al., 1990). However, the wide bandwidth of such designs $(0.05$ to $150 \mathrm{~Hz})$ also allows the mostly low-frequency $(<5$ $\mathrm{Hz}$ ) motion artefacts inherent in large dry electrodes to overwhelm the input range of the amplifier. Dry electrodes have been reported to provide comparable or better biosignals and produce less motion artefact than standard gel electrodes (Searle \& Kirkup, 2000) (Gruetzmann, Hansen, \& Müller, 2007). However, these electrodes have different characteristics and require different amplifier designs than standard $\mathrm{Ag} / \mathrm{Ag}-\mathrm{Cl}$ electrodes. Methods of electronically avoiding power-line interference in an ECG acquisition system have been extensively discussed, simulated and tested in the literature (Winter \& Webster, 1983) (Burke \& Gleeson, 2000) (Spinelli, Pallàs-Areny, \& Mayosky, 2003) (Burke \& Assambo, 2007). What power-line noise remains can be filtered using analog circuitry (e.g. 60-Hz twin-T notch filter) (National Semiconductor, 1969) (Yoo, Kim, 
Song, Lee, \& Kim, 1997) or, as is more commonly done now, by using digital processing techniques (e.g. a finite impulse response notch filter) (Warlar \& Eswaran, 1991)(Rangayyan, 2002). Digital algorithms have an important benefit of being able to deliver a linear phase response throughout the passband, an important characteristic needed to reduce ECG waveshape distortion (Dawson \& Doddington, 1973) (Tayler \& Vincent, 1983). The challenge, in both the hardware and software based approaches, is in aligning the high-Q notch to be and stay exactly at the line frequency, and is solved using frequency tracking techniques.

The most distinguishable part of the ECG waveform is the QRS-complex and so this is what is most commonly used for measuring HR. This complex can be detected using analog circuitry (Burke, 1994) or by using a detection algorithm on the digitally sampled ECG signal (Ruha, Sallinen, \& Nissila, 1997) (Pan \& Tompkins, 1985). Once the QRScomplexes are identified, the heart rate, which is the inverse of the R-to-R interval, can be calculated.

\subsubsection{Goal of This Study}

In this chapter we present a new ECG amplifier design for the Smart Rollator application. The proposed circuit addresses several technical challenges when using grip-style dry electrodes for ECG acquisition during physical activity. These challenges include the mitigation of CM noise in a two-electrode configuration as well as the attenuation of baseline wander. A prototype of the proposed two-electrode ECG system was built, as well as, for purposes of comparison, a conventional three-electrode system. Tests were performed with four subjects, and the quality of the signals acquired compared. 


\subsection{System Theory}

\subsubsection{ECG Signal Quality}

The limb-to-limb ECG signal has a characteristic shape (see Figure 3-1). The amplitude varies from one individual to the next but is generally in the range of $1 \mathrm{mV}$ to $2 \mathrm{mV}$-p for the QRS complex (Webster, 2009). For a healthy individual the ECG reveals information about physiological state. The R-to- $\mathrm{R}$ interval, for example, can be used to calculate heart rate and changes in the amplitude of the QRS-complex can be used to determine breathing rate (Kim, Hong, Kim, Cha, \& Lee, 2007) (Caggiano \& Reisman, 2002).

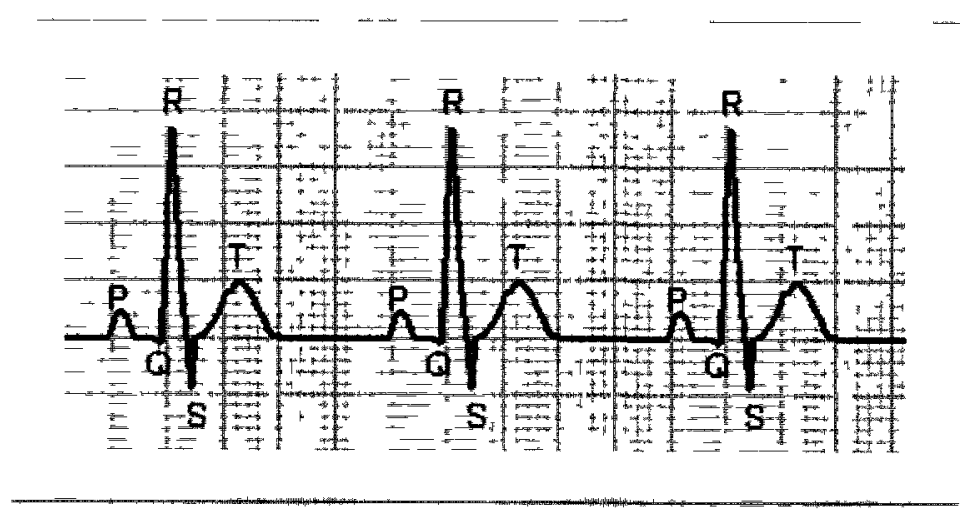

Figure 3-1: The ECG wave for a healthy individual. (Horizontal axis is not to scale.)

In those applications in which the morphology of the ECG waveform is important, such as for clinical diagnosis, the acquisition system must be designed to exhibit low distortion within the bandwidth of the ECG (Dawson \& Doddington, 1973) (Tayler \& Vincent, 1983). That is, the signal path must introduce little frequency dependent attenuation and frequency dependent phase shift.

The American Heart Association, in a report outlining the requirements for clinical ECG monitoring systems, recommends a standardized "spectral" quality for an acquired 
ECG signal intended for diagnostic purposes (Bailey, et al., 1990). The bandwidth, it states, should be limited, using a first order high-pass filter at $0.5 \mathrm{~Hz}$ and a second order low-pass at $150 \mathrm{~Hz}$, the passband response being within $+/-3 \mathrm{~dB}$. The phase distortion introduced by adhering to this guideline would then have a minimal effect on the ECG waveshape.

However, for systems only monitoring heart rate, identifying the R-to-R period may be sufficient. As users maintain balance using the rollator, while standing or walking, we expect the ECG signal will contain a considerable amount of motion related and EMG noises. The highest amplitude noise seen at the lower end of the spectrum is baseline wander, caused by motion artefact. At the upper end of the spectrum one sees power-line interference and muscle activity (EMG). In this case, a narrower bandwidth amplifier could be used, attenuating noise lying in the upper and lower ends of the ECG spectrum.

A secondary goal of our study was to evaluate, qualitatively, the ECG signal achievable on a rollator, both morphologically (i.e. for signal fidelity) and temporally (e.g. for reliable continuous monitoring).

In summary, the ECG signal quality desired is an important factor in the design of an ECG acquisition system. There is an important trade-off to be made between reducing frequency and phase distortion, and reducing noise, particularly in applications in which there is much noise. Acquiring a signal with complete ECG bandwidth would result in a signal which may not be very useful due to high noise content. Reducing the bandwidth to detect only the R-waves (in order to detect HR, HRV and RR), could possibly result in a system with excellent performance. 


\subsubsection{ECG Electrodes}

An ECG skin electrode is typically made of a low-affinity metal coated on one side with a layer of gel electrolyte. Once applied to the skin, there is an electron accumulation (or depletion) which occurs in the metal as a result of ion migration though the electrolyte to (or from) the electrode. The migration occurs to bring the ionic charge and electrical charge at either side of the interface to equilibrium. In this way, the electrode is a transducer revealing electrochemical activity within the human body (Webster, 2009).

When a potential difference is measured across two electrodes, what is being sensed, electrically, is the difference in ion concentration surrounding each of the respective electrodes. This is a dynamic chemical state, and so what is measured is an AC signal.

Wet and dry electrodes differ in their characteristics as sensors, each having advantages and limitations. Wet electrodes rely on the application of a prescribed electrolyte gel which standardizes the performance of the sensor. Dry (gel-less) electrodes rely on the subject's sweat to act as an electrolyte. Gel-less electrodes are preferred for intermittent non-obtrusive sensing applications where skin preparation is not desired.

Dry electrodes may be made of a minimally active metal, such as stainless steel or tin. More recently, a rubber composite materials are being used (Hoffmann \& Ruff, 2007). The characteristics and contact quality with sweat as an electrolyte are not as good as when using a specifically designed electrolyte. The result is that, for the same dimension electrodes, wet electrodes have mostly resistive skin-interface impedance whereas dry electrodes have mostly a capacitive coupling. The magnitude of the coupling impedance, 
for a same contact area, for dry electrodes is typically an order of magnitude higher, or more, than that of wet electrodes (Searle \& Kirkup, 2000).

For the Smart Rollator, in which the natural grip of the user will create a working skin contact and in which the skin fluids (rather than a manufactured gel) will be the electrolyte, a dry electrode design was chosen. The performance of the electrode is expected to depend on the electrode material, as well as its shape and size. It will also depend on measurement conditions, including the quality of the gripping action as well as the quantity of sweat (and of other products) on the user's skin.

\subsubsection{Sources of ECG Noise}

\section{Common-Mode Noise}

The aim of an ECG amplifier is to measure a differential bioelectrical signal. CM noise in this document refers to the unwanted signal in the output of the ECG amplifier resulting from the CM potential at the input electrodes.

\section{Mechanism of common-mode to differential-mode noise conversion}

$\mathrm{CM}$ voltages at the amplifier inputs (with respect to the amplifier circuit ground, not earth ground) can translate into output noise through a number of mechanisms (Huhta \& Webster, 1973), the most significant of these being:

$>$ Instrumentation amplifier (IA) CM rejection: This noise results from the IA's inability to reject the entire CM signal presented at its inputs. The CMRR of the IA specifies the output which will result from the input voltage common on both of its inputs, relative to the IA's circuit ground. Choosing an IA with a large CMRR will minimize this source of noise. Another approach is to have (or let) the 
circuit ground follow the body's CM potential. Doing this will greatly reduce the CM potential at the diff amp inputs (relative to circuit ground), and hence the resulting output noise.

Unbalanced input source impedances: If the skin-to-electrode impedances of the two electrodes are not the same, the imbalance can create a DM signal at the circuit input (Webster, 2009). That is, the CM signal (of the body) will cause a corresponding DM signal (at the amplifier circuit inputs). One approach to minimizing this effect is to design an amplifier with very high input impedance (i.e. input load). The input currents through the dividers will be smaller and the $\mathrm{CM}$ to DM conversion correspondingly smaller.

Unbalanced input load impedances: Similarly, even if the source impedances are identical (as unlikely as this may be in practice), differences in the input load impedances of the inputs will result in a differential signal at the circuit input terminals. The solution is to have very large input impedances (so that the input currents are small), or else to have well matched input impedances.

The above three effects contribute to the total "effective" CMRR of the ECG amplifier (Thakor \& Webster, 1980) (Winter \& Webster, 1983).

\section{Sources of CM noise}

The main source of CM noise is power-line coupling. The body, being capacitively coupled to both power lines and ground forms a voltage divider, the result being a significant line-frequency CM signal in the body (see Figure 3-9). The amplitude is typically 1 to 10 volt peak-to-peak, relative to earth ground(Thakor \& Webster, 1980; 
Winter \& Webster, 1983). Interestingly, changes in coupling between body and ground (e.g. while walking), will change one leg of the voltage divider, the result being a modulation of the CM noise.

If the person is electrically isolated from earth ground, a static (DC) charge may also be present. The magnitude of this charge can be from millivolts to thousands of volts.

Other sources of CM noise include electrical or magnetic coupling to florescent lighting and nearby large motors. In practice, the CM potential consists mostly of DC components, power-line frequency and power-line frequency harmonics.

\section{Differential-Mode Noise}

DM noise, in this document, refers to the undesired part of the signal, the signal being the potential difference between the two input electrodes. The most significant sources of this type of noise include muscle activity (EMG), movement of the electrodes on the skin (motion artefact) and DM power-line interference.

\section{Electromyographic Activity}

Activity by muscles located between bioelectrodes will result in a differential EMG signal being acquired. An EMG signal, when acquired with electrodes mounted on the skin surface, has a bandwidth of about 20 to $450 \mathrm{~Hz}$ (van Boxtel, 2001). This overlaps with that of the ECG, which has components from about 0.05 to $150 \mathrm{~Hz}$ (Bailey, et al., 1990).

The amplitude of the EMG noise depends on both the closeness of the muscles to the electrodes as well as the strength of the muscle activity. However, as the magnitude of 
this noise is typically less than or about the same as that of the ECG signal, it is not a likely cause of saturation within the ECG amplifier.

\section{Motion Artefact}

Motion artefact refers to undesired signal changes resulting from movement of the electrode or the subject (or both) (Webster, 2009).

For dry electrodes, the most significant effect is from a change in pressure of the electrode against the skin. As the electrode (or parts of it) move towards or away from the skin (resulting in a thinner or thicker electrolyte layer separating them), the capacitance of the skin-to-electrode interface changes. A change in capacitance with a same total charge on the plates (short term) causes a change in potential (short term). For a gripstyle electrode, changing the strength of the grip is an example of what may create this effect.

A different effect is observed when there is a change in contact area (i.e. a change in the total surface area in which there is electrolyte between the electrode and the subject's skin). This will cause a corresponding change in impedance; larger contact area will result in a smaller impedance (both capacitive and resistive), and vice versa. A change in contact area, for a grip-style electrode will result mostly from how completely the electrode is being gripped and to a lesser extent by the tightness of the grip.

\subsubsection{Management of ECG Noise}

\section{Power-line Interference}

This is the highest amplitude noise typically found in an ECG monitoring system (Webster, 2009). This noise, being within the bandwidth of the ECG signal, has obtained 
much attention by researchers. Many approaches of avoiding line noise coupling, of reducing its transformation into a DM signal and of attenuating this noise by filtering have been (and are still being) developed (Warlar \& Eswaran, 1991) (Huhta \& Webster, 1973).

To reduce possibility of saturation of the ECG amplifier by CM power-line interference, popular approaches include:

$>$ Using a high-voltage power supply and amplifiers. Using this approach, so long as the input range of the IA inputs is large enough to accommodate the unattenuated power-line coupled signal (i.e. up to 20 to 30 Volts peak-topeak), saturation can be avoided. An IA with a very high CMRR is then used to subtract the $\mathrm{CM}$ signal from the underlying $2 \mathrm{mV}$ peak-to-peak ECG signal.

$>$ Shunting the subject's body to earth ground. This is a popular technique if done safely (Webster, 2009), though practical only in applications in which earth ground is available to the system (generally, not the case for portable systems).

$>$ Portable and battery powered systems typically take a slightly different approach in which the circuit ground is made to track the subject's CM body potential. This can be accomplished by either using a third (driven) feedback electrode (Winter \& Webster, 1983), or by creating a CM shunt from the electrode inputs to circuit ground (Dobrev, Neycheva, \& Mudrov, 2008).

Power-line interference within the DM ECG signal must be managed using different techniques than for CM power-line interference. Fortunately, this type of noise is both fixed frequency and stationary (or almost). As such, it is suitable for using digital 
techniques for its removal, with little or no phase distortion to nearby frequencies in the ECG signal. Numerous adaptive and FIR filtering methods have been proposed for doing this.

A major requirement of the ECG amplifier is to provide a minimally distorted signal to the processor. As long as the DM signal is less than the full-scale amplitude of the signal path (typically between $2.5 \mathrm{mV}$ and $10 \mathrm{mV}$, referred to the input of the ECG amplifier), noise processing in software is effective. For those periods where the differential signal amplitude exceeds the signal range and clipping occurs, software techniques can be used to identify these regions and calculate which parts of the postprocessed signal will be distorted as a result of the clipping.

\section{Offset and Baseline Wander}

DC offset is due to (1) the difference (i.e. the imbalance) in the charges of the capacitors inherent in the electrode-to-skin interfaces, and (2) the difference (i.e. the imbalance) in the Nernst potential of the electrode-to-skin interfaces (between both electrodes). Baseline wander results from a slow change in the DC offset potential across the electrodes.

When one (or both) of the electrodes changes its capacitance (e.g., due to the change in the effective area of contact between the electrode and the skin, or the average distance between the electrode and the skin), the charges on this "capacitor's" plates will create a new potential. This change in potential manifests itself as baseline wander and a new DC offset potential. 
The Nernst potential may change as a result of a number of effects, such as the subject sweating more, and the ionic content or concentration changing, or by the film of ions surrounding the electrolyte-electrode interface being disturbed. A change in this potential also shows up as baseline wander.

DC offset and baseline wander are low frequency DM types of noise (typically $<1$ $\mathrm{Hz}$ ). The amplitude of these can be relatively high (tenths of a Volt to several Volts are quite typical), and so must be attenuated to at least a level below the supply rails prior to reaching any active components. This can be accomplished quite well with a low-order passive high-pass filter within an "input conditioning" stage prior to any active amplification. Further filtering with DSP, following conversion, can further attenuate this noise with little distortion.

\section{Electromyographic Activity}

Whereas the spectral power content of an EMG is mostly found in the $20 \mathrm{~Hz}$ to $150 \mathrm{~Hz}$ frequency range, an ECG has most of its power in the 5 to $25 \mathrm{~Hz}$ spectrum ( $\mathrm{Lu}$, et al., 2009) (Redfern, Hughes, \& Chaffin, 1993). By carefully choosing the low-pass filter's cut-off frequency and order, most EMG noise can be removed from the ECG signal with minimal distortion (Thakor, Webster, \& Tompkins, 2007).

The amplitude of the EMG signal in a limb-to-limb ECG monitor has, at most, a peak-to-peak amplitude similar to that of the ECG signal. Thus, the EMG will not normally, in itself, cause clipping of the ECG amplifier, even in a low voltage system, and so can be either filtered by software (following digital conversion) or in hardware (immediately following the differential amplifier). 


\subsubsection{Power Consumption}

The Smart Rollator is a battery operated data acquisition system embedded within a rollator device. It should have the ability to run for days or weeks on a battery charge, and each subsystem (power supply, ECG amplifier, speed-distance monitor, digital processor, etc.) must be small and light enough to mount onto the rollator without affecting its ease of operation.

The main considerations in reducing power consumption in an ECG amplifier design include:

\section{Using a Low Voltage Power Supply}

Since power consumption is typically proportional to the square of the supply voltage, significant reductions in energy requirements are possible by minimizing supply voltages. However, having low supply rails also means that throughout the signal path care must be taken to avoid the signal's peak exceeding the input range of the active elements (IAs, OAs, and $\mathrm{ADCs}$ ). In such designs, using components rated for operation from "rail-torail" on both inputs and outputs becomes a necessity in order to avoid clipping of the signal.

\section{Choosing Low Power Active Components}

A good selection of high performance op amps and instrumentation amplifiers are now available based on CMOS technology. These have very low power needs, but at the cost of greatly reduced gain bandwidth product. Multiple stages may be needed to get even modest amplification and filter orders. 
Reducing Average Current Use in Passive Components

Equally important is designing the circuit such that average current in the paths through passive components is low. Choosing high impedance op amp feedback loops and passive filters with high $\mathrm{R}$ and $\mathrm{C}$ values, for example, reduces current consumption. This may impact circuit performance (it will be more susceptible to noise, for example) so reasonable compromises are necessary.

\section{Consider Powering Down Circuitry between Measurements}

If only intermittent operation is needed, some or all of the circuit can be turned off when not needed. Here, power-up and settling time must be taken into account to asses if this is a feasible option. If start-up time plus acquisition/processing time is significantly less than the required period of sampling then there is benefit.

\subsubsection{Conventional Three-Electrode ECG Amplifier}

A typical three-electrode ECG acquisition system, shown in Figure 3-2, consists of two input electrodes and a driven reference electrode (seen at the left side of the diagram). The input electrodes feed into an ECG amplifier (the lightly shaded section of the diagram), which converts the differential signal to a voltage suitable for digital conversion (right side of diagram). In this chapter we look at a new ECG amplifier design suitable for use in the Smart Rollator, and compare it to a conventional three-electrode amplifier similar to what is shown here.

The voltage difference between the two electrodes is converted to a ground referenced signal by a differential amplifier. However, prior to this, an input conditioning stage is used to attenuate those frequencies components which are out of the bandwidth 
of interest and which may cause the amplifier to saturate. Further amplification and bandpass filtering prepare the signal for analog-to-digital conversion. Still further filtering and signal processing is done digitally, following conversion. The goal of the analog filtering is to avoid clipping of the signal by out-of-band noise prior to conversion and to perform any required anti-aliasing.

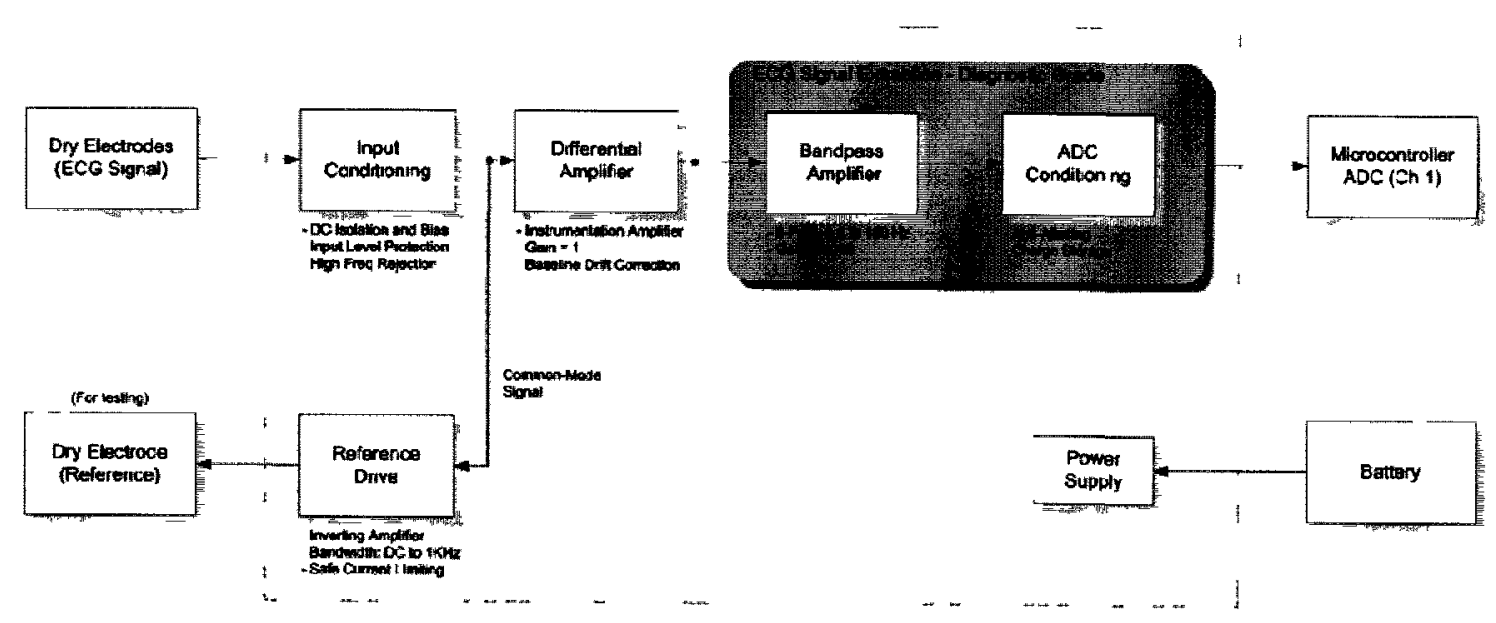

Figure 3-2: Dry electrode ECG amplifier with optional driven reference electrode

The average of the input electrode voltages (i.e. the CM signal) is amplified, inverted and used to drive the body potential via a third electrode. This creates a feedback loop in which the result is that the body potential is brought to that of the ECG amplifier's ground. The advantage is twofold: the instrumentation amplifier sees little or no CM signal (relative to circuit ground), effectively raising the CMRR of the IA. More importantly, the CM potential (relative to circuit ground) is kept well within the supply rails so that instrument amplifier input saturation (due to $\mathrm{CM}$ noise) is avoided.

The power supply in a typical portable application provides a clean bipolar supply from a battery pack or single cell. 


\subsection{System Design}

\subsubsection{Technical Goals of the Design}

The goals in designing an ECG amplifier for the Smart Rollator can be summarized as follows:

To amplify the small differential ECG signal sensed by the electrodes to a level suitable for digital conversion. The ECG signal (Figure 3-1) typically has a magnitude of about 1 to $2 \mathrm{mV}$ peak-to-peak. The input range of the analog-to-digital-converter (ADC) depends on the part chosen and its configuration, but is typically in the $3 \mathrm{~V}$ to $10 \mathrm{~V}$ full scale range. For the ADCs integrated into low-power microcontrollers (such as the STM32, discussed in Section 2.4), the input range is typically $3 \mathrm{~V}$. Hence an in-band gain of approximately 1000 to 2500 would be required.

To be designed for operation with grip-style dry electrodes, mounted onto or embedded into the rollator handles. Amplifier specifications should take into account the characteristics of large dry electrodes (such as large capacitive coupling).

To operate without the need for a reference electrode. That is, to obtain a good quality ECG signal through only the two handlebar electrodes the subject holds onto during normal use of the rollator. Similar applications, such as stationary bicycles, have both an input electrode and a reference electrode on each of the handles. For the Smart Rollator, ensuring a particular grip is more difficult, and so a two-electrode setup is preferable. 
$>$ The signal should, at a minimum, be suitable for heart rate measurement and avoiding signal clipping (i.e. have good baseline management) during moderate activity (e.g. walking slowly with the rollator). Clipping will significantly affect what can be accomplished with digital signal processing (DSP).

Achieving days to weeks of operation on a small battery supply. The ECG monitoring subsystem is one of many in the Smart Rollator system, and is intended to be run off a common, compact, power source (likely, a small rechargeable battery pack). Although this is a requirement difficult to quantify, our aim in designing the ECG amplifier was for it to operate from a single-ended $3 \mathrm{~V}$ supply and its average current consumption to be below 2 mA.

Again, the approach taken for this design was to perform a minimal amount of noise filtering in the electronics (just enough to avoid saturation during typical operation) and then to perform signal processing digitally, where there are more choices, where better performance is achievable and where the configuration is easier to change. Digital signal processing of ECG waveforms is a well researched area (Pan \& Tompkins, 1985) (Rangayyan, 2002) and so was not within the scope of the present research.

\subsubsection{Choice of Electrodes}

A requirement is that the Smart Rollator use dry grip-style electrodes. This type of electrode is not readily available, so a set of prototype electrodes were built and their characteristics measured. Tin (Sn) was chosen as the electrode material due to its low 
toxicity, it being corrosion-resistant, and its availability. Small sheets of tin-plated steel were wrapped around the handles of the rollator and wired to the amplifier.

Other alternative materials for constructing the electrodes include stainless steel, and now, rubber composite materials. Although these have some differences in characteristics from tin, it was considered out of scope for our study to perform a detailed comparison among various types of dry electrodes. As most bioamplifier studies are with popular wet electrodes, it is the major differences between larger dry electrodes and these smaller wet $\mathrm{Ag}-\mathrm{AgCl}$ electrodes that would be most relevant. So as a first step in the design of the ECG acquisition system, the impedance of these two types of electrodes where compared.

\subsubsection{Power Management}

To minimize power consumption and to simplify the power supply design (by operating from the same low voltage supply as the proposed Smart Rollator core), a simple 3-Volt supply was used. The prototype amplifier was built with a small 3V lithium coin cell for its supply (see Appendix A.4 for specifications).

If sharing a power source (such as battery pack) with other modules in the Smart Rollator system, the ECG amplifier's V- supply input would be connected to system ground, and the V+ supply input to the battery source. Of course, appropriate supply decoupling between digital and analog systems would also be needed.

The signal output, with this configuration, would be biased at $\mathrm{V}_{\text {SUPPLY }} / 2$, with a range from $\mathrm{V}$ - to V+. This range should work well with most ADC's, as these can usually operate with an input range of ground to $\mathrm{V}_{\mathrm{CC}}$. 


\section{Choice of Components}

As a general rule, to keep current consumption down, passive components (resistors and capacitors) in the amplifier circuit were chosen such that their peak current did not exceed $100 \mathrm{uA}$. CMOS-based instrumentation amplifier (IA) and op-amps (OAs) were chosen for their very low power consumption and their ability to operate down to $2.5 \mathrm{~V}$ (see Appendices A.2 and A.3, respectively, for specifications).

When operating from a low voltage power supply (in this design, nominally at $+/$ $1.5 \mathrm{~V}$, and extending down to $+/-1.25 \mathrm{~V}$ as the battery discharges), choosing active components (IA and OAs) with rail-to-rail ranges at their inputs and outputs was essential. The parts chosen also had input clamping and latch-up prevention, important for reliable operation.

One limitation of linear CMOS amplifiers is their very low gain-bandwidth product (GBWP) when operating at low voltages. The OA chosen for this design (the Texas Instruments TLV2404) had a GBWP of $55 \mathrm{~K} \mathrm{~Hz}$, limiting the stability of high frequency signals in high gain stages. Amplifier configurations for this design were therefore limited to 100 per stage and signals were passively low-pass filtered first. Also, as SallenKey topology filters, as a rule of thumb, require at least 100 times the high side of the signal bandwidth (Baker, 2003), these were avoided altogether in the design.

\section{Power Cycling}

If only periodic ECG measurements are required, average power consumption of the amplifier could be considerably reduced by turning off its supplies when not needed. This activity would be controlled by the power management subsystem of the Smart Rollator 
system under control of the processing subsystem. Testing such a strategy to evaluate the power savings was considered out of scope for this study.

\subsubsection{Noise management}

A key goal of the amplifier design was to manage noise so as to not clip at any point in the signal path up to (and including) digitization. A competing goal was to not introduce more phase (or other) distortion than what is needed (e.g. from aggressive analog filtering). By avoiding signal clipping, any additional filtering needed to isolate the ECG components of interest (in our case, the R-wave) could be performed with digital processing (i.e. DSP filtering and/or algorithmic methods).

\section{Power-line Interference}

If the body's CM potential is kept at or near the amplifier circuit's ground potential, then the IA will "see" less of a CM signal, magnifying the circuit's overall immunity to the CM signal (its "effective" CMRR). A conventional three-electrode circuit does this by injecting a current into the body such that the CM signal at the amplifier inputs tracks the circuit ground potential. The proposed two-electrode circuit accomplishes this in another way: by the addition of a "CM shunt" circuit at the input of the amplifier, similar in design to what is used in subscriber line telephone systems (Dobrev \& Daskalov, 2002). Tracking of CM potential to circuit ground also prevents saturation of the active components.

The amount of power-line interference in the ECG signal will be related to the amplifier circuit ground to earth ground coupling. That is, if there is weak coupling impedance between the circuit's ground and earth ground, there will be proportionally 
less electrode current and so, (1) less signal attenuation due to the electrode impedance, and (2) less CM-to-DM noise from unbalanced electrode impedances and/or amplifier input impedances.

\section{Motion Artefact (DC Offset and Baseline Wander)}

In this design, motion artefact is managed by:

$>$ Including a first order 5-Hz high-pass filter prior to any amplification, reducing occurrences of saturation in the IA and later amplification stages.

$>$ Adding buffers (U1B \& U1C) near the inputs, to lower the signal impedance, so that passive filtering can occur before any amplification occurs. As the circuit ground is tracking the body's CM potential, these buffers are unlikely to saturate.

Signal clamping circuits, used to hasten the recovery time following a quick DC offset shift. The clamp shunts the current from a preceding blocking capacitor such that the signal voltage is kept within $100 \mathrm{mV}$ of circuit ground. Clamping CM DC shifts is performed by Schottky diodes D1 to D4, at the input stage. Clamping of DM DC shifts is performed by diodes D4 and D5, just prior to the final stage.

\section{EMG Noise}

Though most of the EMG frequencies are beyond the bandwidth of the ECG, there is some overlap. The design trade-off is the attenuation of most high-frequency noise energy while not introducing significant distortion in the ECG passband. A high order 
filter to block most or all EMG frequencies (with a cut-off frequency of around $40-\mathrm{Hz}$, for example) could be used if ECG morphology is not intended.

As EMG noise does not, generally, have amplitude much greater than that of the ECG signal, signal clipping due to EMG is unlikely. For this reason, implementing the EMG low-pass filter in DSP is used in this design. In addition, a second order $150 \mathrm{~Hz}$ low-pass filter (implemented in hardware, primarily for ADC anti-aliasing) also attenuates some of the higher frequency EMG components.

\subsubsection{The Proposed Two-Electrode ECG Amplifier}

Figure 3-3 is the schematic of the designed two-electrode amplifier. An input stage conditions the signal prior to being introduced to the IA. The IA extracts and amplifies the differential signal, and then it is further filtered and amplified in preparation for digital conversion. The power supply splits a $3 \mathrm{~V}$ battery source into a clean $+/-1.5 \mathrm{~V}$ supply.

The DC blocking capacitors at the input eliminate DC offsets at the input, which could otherwise saturate U1. Low-frequency components of the signal are further eliminated following the buffers (U1B and U1C), with a first-order 5-Hz high-pass filter. Since low frequency noise (motion artefact) can be tens to hundreds of millivolts in magnitude, high-pass filtering is performed before any signal amplification.

The CM shunt circuit implemented here (consisting of U1D, U1A and related components) presents a low-impedance path to circuit ground (Dobrev, Neycheva, \& Mudrov, 2008) at the points just following the DC blocking capacitors. However, it presents high DM impedance, resulting in an ECG signal at the buffer inputs 


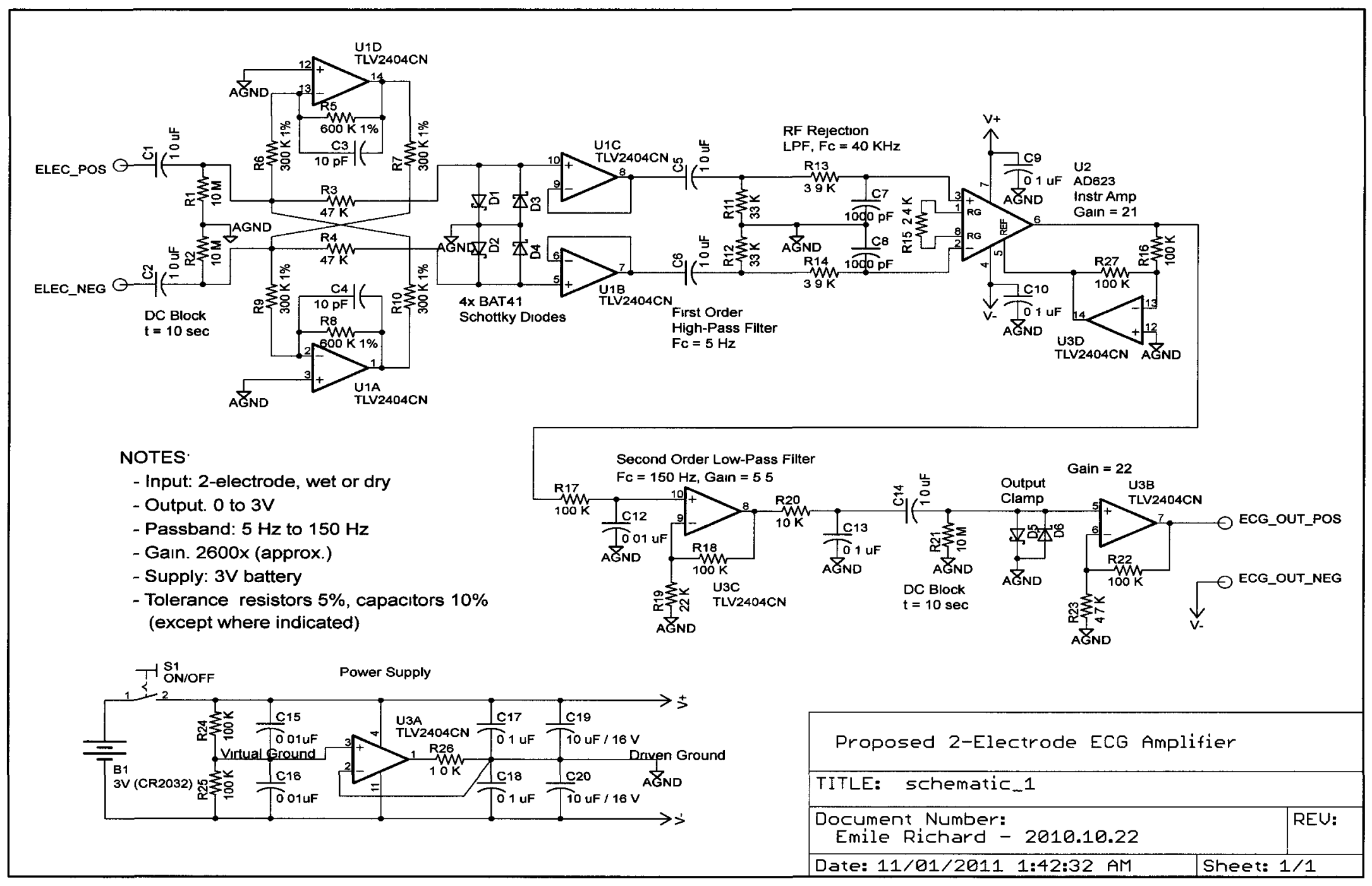

Figure 3-3: Proposed two-electrode ECG amplifier 
(pins U1C-10 and U1B-5) having little attenuation. Clamping diodes D1 to D4 speed up the removal of excess charge from the input blocking capacitors to circuit ground (via current-limiting safety resistors R3 and R4), in the event of a quick (and greater than $+/-$ $100 \mathrm{mV}$ ) amplitude changes at the electrode inputs.

Note that the inputs of the IA and OA's all have integrated protection diodes. The external clamping diodes (D1 to D6) are intended solely, as just mentioned, to speed recovery of the ECG signal back into the linear operating region.

The buffers (U1C and U1B) lower the differential signal's impedance prior to it being put through a balanced passive high-pass filter (to attenuate some of the motion artefact) and then through a balanced passive low-pass filter (this used to snub RF frequencies which may cause performance issues within the IA).

The IA (U2) is programmed, with R15 for a gain of about 40. This gain is reduced to 20 by U3D, by forcing the reference level of the output stage of the IA in the reverse direction of the output signal. This makes better use of the output range of the IA. The output of the IA (where the QRS amplitude is now about $20 \mathrm{mVpp}$ ) is put through two first order $150-\mathrm{Hz}$ cutoff low-pass filters for anti-aliasing in preparation for digital conversion, and then through another amplification stage (U3C, gain of 5.5). The signal is DC blocked again, this time to eliminate any DC offset caused by the amplifying elements (U2, U3D and U3C).

At this point (pin U3B-5), the QRS complex has a magnitude of about $100 \mathrm{mVpp}$. A pair of Schottky diodes clamps this signal to about $+/-100 \mathrm{mV}$ (i.e. $200 \mathrm{mVpp}$ ), to allow for quick recovery of C14's charge in the event of a significant DM noise peak which has made its way to this point. A final gain stage buffers the signal in preparation for 
conversion, while bringing its amplitude to a level compatible with the ADC's input range.

The power supply circuit splits the battery supply, creating a mid-point voltage for use as an analog ground. This reference potential has $\mathrm{C} 17$ through $\mathrm{C} 20$ supplying the immediate current needs of the circuit, with U3A injecting additional current, as needed. R26 maintains stability of the op-amp control loop.

\subsubsection{The Reference Three-Electrode ECG Amplifier}

Figure 3-4 is the schematic of a newly-designed but typical three-electrode ECG amplifier. An IA extracts and amplifies the differential signal presented at the electrode inputs. This is followed by further amplification and bandpass filtering prior to being output to an ADC. A feedback circuit amplifies and inverts the input CM potential, driving a reference electrode. A power supply splitter creates the necessary $+/-1.5 \mathrm{~V}$.

The input signal from the electrodes are low-pass filtered (via $\mathrm{R} 1 / \mathrm{C} 1$ and $\mathrm{R} 2 / \mathrm{C} 2$ ) to attenuate any RF components in the signal prior to being fed into an IA (U1). This filtering is recommended by the IA manufacturer to prevent artefacts from occurring due to nearby mobile phones or other RF transmitting devices. The $47 \mathrm{~K}$ resistors also limit the input currents to safe levels should there be a circuit fault.

The IA is programmed, via R3 and R4, for a gain of about 20. A feedback op amp on its output (U2D) integrates the output and presents it back to the IA's output stage reference input (pin U1-5). This effectively creates a first order high-pass filter with a cutoff of 5-Hz, attenuating DM DC offset and baseline wander.

The IA output (pin U1-6) is further amplified as well as band limited with a second 


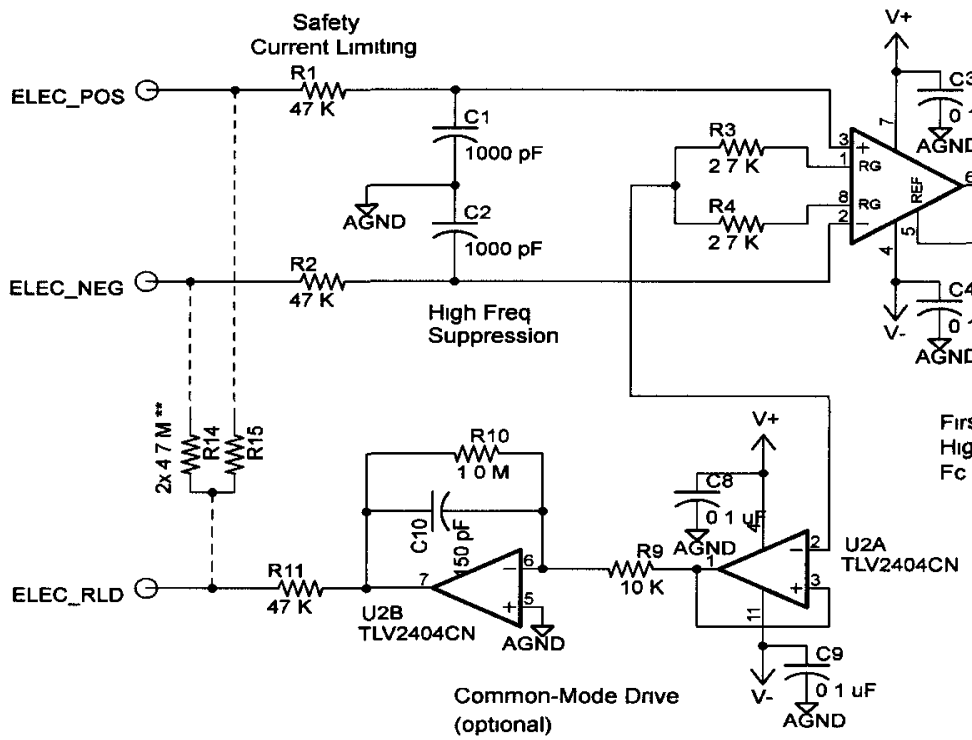

NOTES:

- Input. 2-electrode, wet or dry

- Output: 0 to $3 \mathrm{~V}$

- Passband: $5 \mathrm{~Hz}$ to $150 \mathrm{~Hz}$

- Gain: 2000x (approx.)

- Supply: $3 V$ battery

- Tolerance: resistor $5 \%$, capacitors $10 \%$

** If reference electrode is not used, bridge the

ELEC_RLD output to ELEC_POS and ELEC_NEG

inputs with $4.7 \mathrm{M}$ resistors

Second Order Low-Pass Filte $F C=150 \mathrm{~Hz}$, Gain $=100$

U2C

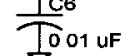

A

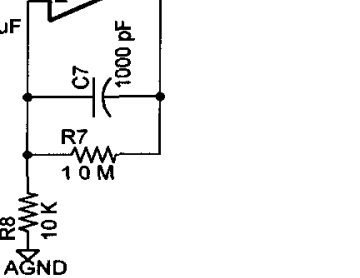

岂

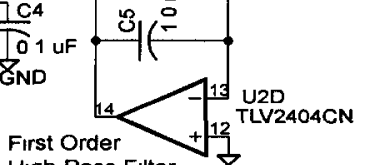

High-Pass Filter

$\mathrm{C}=5 \mathrm{~Hz}$

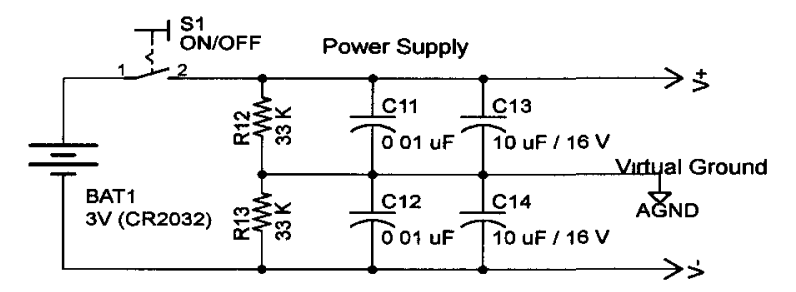


order low-pass filter having a cutoff frequency of $150 \mathrm{~Hz}$. The total gain of the circuit is about 2000, bringing a QRS complex, typically around $1 \mathrm{mV}$-p at the amplifier inputs to about $2 \mathrm{~V}$ p-p for digital conversion.

The CM voltage of the input signal is sampled from the mid-point of the gain resistor (R3 and R4). This value is inverted, amplified, band-limited and then presented back to the body through a safety current-limiting resistor. The net effect of this control loop is that the body is driven with a small current such that its CM voltage becomes close to circuit ground potential.

\subsubsection{Grip Electrode Description}

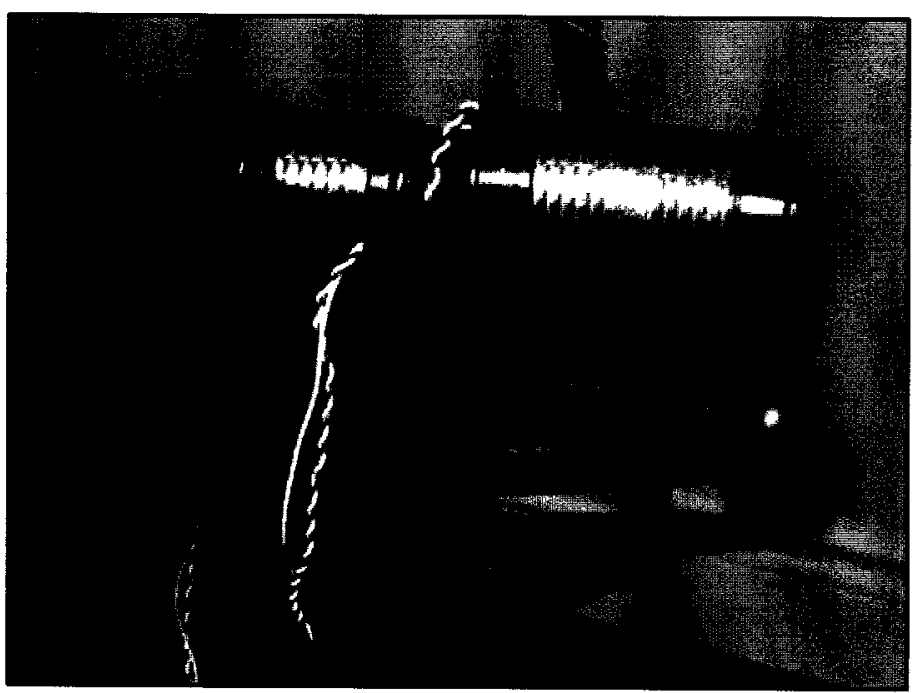

Figure 3-5: Rollator handle equipped with the prototype dry electrode and "thumb" electrode.

The rollator was equipped with dry grip electrodes (see Figure 3-5) similar to the set built for characterization (Figure 3-6). Two $12 \mathrm{~cm} \mathrm{x} 8 \mathrm{~cm}$ sheets of tin-plated steel were wrapped around the handles of the rollator and wired to the amplifier assembly. The length of the electrodes were close to that of the handles themselves, so a normal grip on 
the rollator handles would result in a large contact area between the user's hands and the electrodes. A "thumb electrode" was added to each handle to allow for testing of the three-electrode (conventional) amplifier circuit.

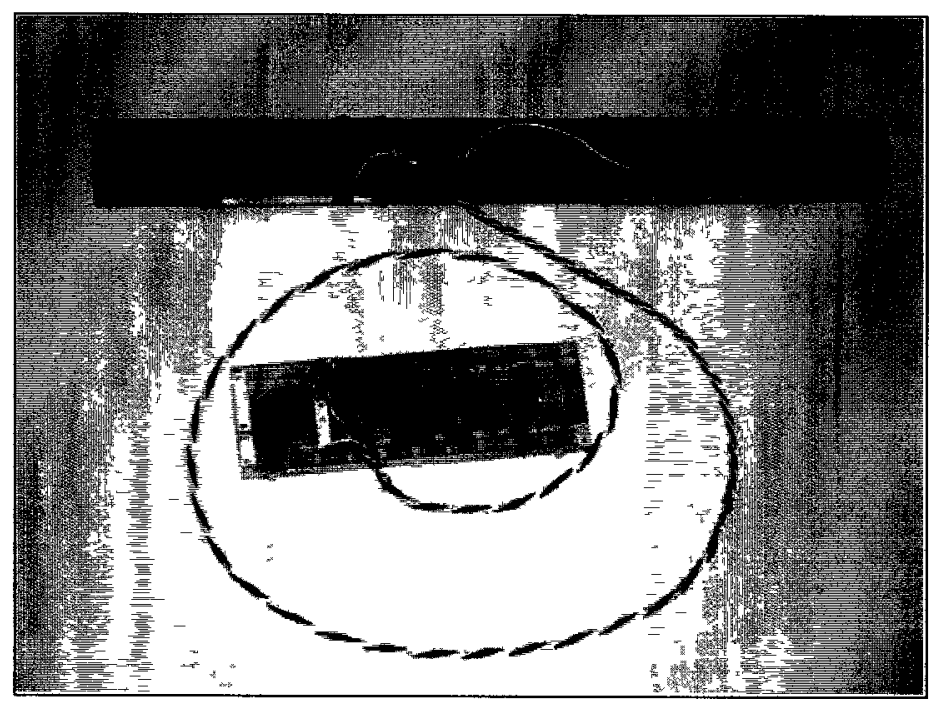

Figure 3-6: Original prototype of ECG circuit with grip-style dry electrodes.

Related to the choice of electrodes was their wiring and susceptibility to EM interference. The loop formed by the user, the electrodes and the wires connecting them to the circuit is a source of DM EM interference. Any changing magnetic field going through this loop, such as ambient power-line EMI, would generate a small current across the amplifier inputs. To minimize the effective loop size, a ground wires were twisted around the electrode wires (Huhta \& Webster, 1973).

\subsection{Electrode Characterization Tests}

To better understand the characteristics of dry grip-style electrodes, prototype electrodes were built (as shown in Figure 3-6) and tested. As a comparison, popular paste-on wet $\mathrm{Ag}-\mathrm{AgCl}$ electrodes were also characterized. 


\subsubsection{Methods}

\section{Setup}

A bioimpedance analyzer (BIA), consisting of a Solartron 1294A Impedance Interface connected to a Solartron 1255B Frequency Response Analyzer, was used to estimate the spectral impedance of the skin-to-electrode interface (hereafter referred to as the electrode impedance) of the dry grip electrodes and, for comparison purposes, conventional paste-on $\mathrm{Ag}-\mathrm{AgCl}$ wet electrodes (see Appendix A.1 for specifications). The BIA was connected in the two-electrode configuration to measure the total path impedance, including the impedances at both skin-to-electrode interfaces as well as the limb-to-limb internal body impedance. As the internal body impedance is several orders of magnitude smaller then the electrode impedance, it was ignored in calculating electrode impedance.

The analyzer was programmed with the vendor supplied software (SMaRT Impedance Measurement Software, v3.0.1) on a host PC to perform measurements at frequencies spanning the range of $1 \mathrm{~Hz}$ to $100 \mathrm{~Hz}$ (5 data points per decade, for a total of 11 measurements for each test). For safety reasons, the peak excitation current was set to 100 uA. Each test run, due to the equipment's internal averaging process, took a total of about 8 minutes to complete.

\section{Procedure}

A test subject is asked to hold onto the metal grip electrodes using a "comfortable moderately tight grip". The BIA program is run, measuring and recording, for the requested frequencies, the total impedance between the electrodes. On finishing, the 
subject removes their hands from the grips. This first test is labelled "dry electrode, run $\# 1 "$. The subject then holds the electrodes again and the test is repeated ("dry electrode, run \#2").

The same BIA program is then used to measure the total impedance using wet electrodes. A new set of paste-on $\mathrm{Ag}-\mathrm{AgCl}$ electrodes are pasted to the inside of the subject's wrists and the BIA test is run. These results are labelled "wet electrode, run \#1". Without removing the electrodes the test is repeated ("wet electrode, run \#2").

Data are plotted using MATLAB; one figure comparing the impedance magnitudes for the dry to wet electrodes, and another comparing the impedance phase for the electrodes. Again, the magnitude values plotted are actually the total path impedances, with no data conditioning performed, so the actual skin impedance at each electrode is about half of the plotted value.

\subsubsection{Results/Analysis}

A volunteer was recruited and consented to take part in this test. This research was reviewed and approved by the Carleton University Research Ethics Board.

Figure 3-7 shows the results of these tests. The left plot indicates that the dry electrodes tested (data points plotted with triangles) had consistently lower skin impedance than the wet electrodes tested (plotted with circles). At $1-\mathrm{Hz}$ the dry electrodes had about half the impedance of the wet electrodes $(100 \mathrm{~K}$ Ohm for the wet electrode vs. $50 \mathrm{~K}$ Ohm for the dry electrode). At $100-\mathrm{Hz}$, the dry electrodes were almost an order of magnitude lower in impedance $(45 \mathrm{~K} \mathrm{Ohm}$ for the wet vs. about $6 \mathrm{~K} \mathrm{Ohm}$ for the dry). 

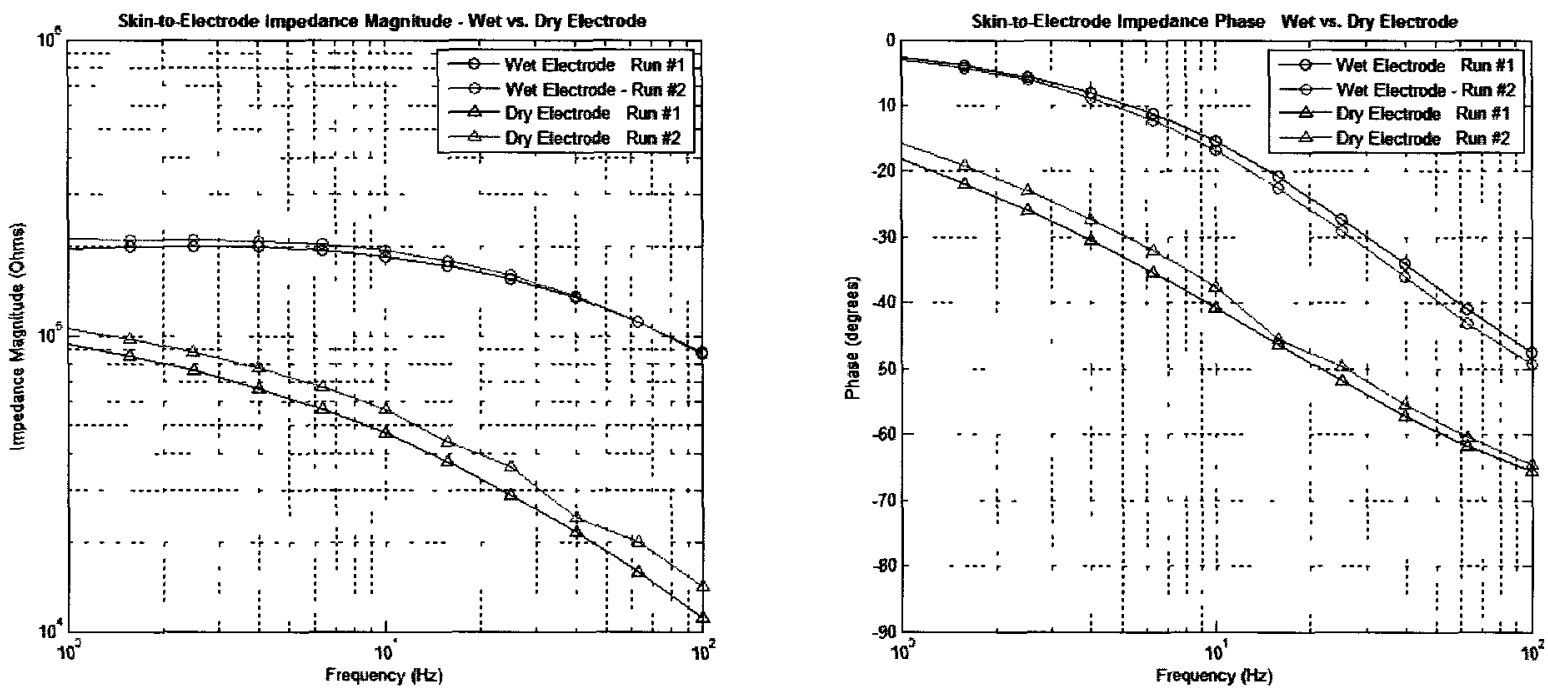

Figure 3-7: Skin-to-electrode interface impedance for popular wet Ag-AgCl electrodes (circles), and large dry grip-style tin electrodes (triangles). Impedance magnitude is plotted on the left, phase lag on the right.

Comparisons by other researchers of dry metal plate electrodes to wet $\mathrm{Ag}-\mathrm{AgCl}$ electrodes showed that for the same size electrodes, wet electrodes have significantly lower impedance (Searle \& Kirkup, 2000). A likely explanation for our results is that the large skin contact area of the grip electrodes is a major factor in achieving the low electrode impedance. The dry electrodes had an active exposed area of about $60 \mathrm{~cm}^{2}$ $(12.2 \mathrm{~cm} \times 5.1 \mathrm{~cm})$ each, of which about $75 \%$ had contact during the test. The wet electrodes, by comparison, had an active area of about $0.3 \mathrm{~cm}^{2}(1.12 \mathrm{~cm}$ diameter "button"). The user having some grip pressure on the handles may also have made for close electrode contact, further contributing to the observed low impedance.

The impedance phase plot (Figure 3-7, right plot) shows that the dry electrodes tested had a larger phase lag than the wet electrodes, also suggesting that the dry electrodes have a greater relative contribution to their impedances by capacitive coupling than do the wet electrodes. At around $15 \mathrm{~Hz}$, for example, the dry electrode have about half of 
their impedance contribution from a capacitive coupling (i.e. there is a 45 degree phase lag). The wet electrodes, however, have half of their impedance from capacitive coupling only at around $80 \mathrm{~Hz}$.

This lower electrode impedance means that the dry electrodes will have a good immunity to power-line (and other) CM body noise being converted to DM noise due to electrode impedance mismatch (Huhta \& Webster, 1973). It also means that there will be a smaller (albeit only a slightly smaller) voltage drop across this impedance and so a larger signal at the ECG amplifier inputs.

However, the larger capacitive coupling of the dry electrodes also means that they will be more susceptible to baseline wander as a result of motion. This is because if there is a change in the average skin-to-electrode distance, there will be a corresponding change in capacitance and a corresponding change in the DC potential across the electrode. The sum of the DC potential changes on both electrodes is what we observe as "baseline wander".

Since the capacitance is large in this type of electrode, so too will the amplitudes of the baseline changes be. An ECG monitoring system using such electrodes will therefore need to effectively manage this low frequency noise, in order (a) to avoid clipping of the DM signal as it gets amplified, and (b) to not distort the shape of the ECG wave.

This motion artefact, when the user is walking slowly and their balance is shifting slowly from the left to right handles, is, fortunately, mostly in frequencies at the lower end or below the region of interest of the ECG spectrum (the region of interest being about $4 \mathrm{~Hz}$ to $40 \mathrm{~Hz}$ ). Hence, so long as this low frequency DM noise is sufficiently attenuated such that it does not contribute to saturation of the IA or OAs, and so long as 
this noise remains in a spectrum below those frequencies of interest in the ECG, it can be effectively managed using DSP techniques.

\subsection{ECG Amplifier Characterization Tests}

\subsubsection{Methods}

\section{Input Impedance Measurement}

The Solartron 1294A Impedance Interface, connected to the Solartron 1255B Frequency Response Analyzer, was once again used for spectral impedance measurement, this time to measure the input impedance of the designed ECG amplifier. The DM impedance was tested by "floating" the circuit while measuring the current into and voltage across the amplifier inputs, while being fed a $1 \mathrm{mV}$ p-p sine wave. The CM impedance was tested by shorting the electrode inputs and then taking a measurement between this point and the circuit's ground.

This equipment was programmed, with the vendor supplied software, to take measurements for the frequency range of 1 to $100 \mathrm{~Hz}$ (10 readings per decade, for a total of 21 data points). The test was run once with the DM setup and once with the CM setup. The DM and CM tests were then repeated but this time with the reference three-electrode ECG amplifier (the drive electrode was left unconnected). Each test run took a total of about 14 minutes to complete.

MATLAB was used to plot the results. No additional processing of data was performed. 


\section{Frequency Response and Spectral CMRR Measurement}

Spectral gain measurements were made using the Solartron 1255B Frequency Response Analyzer (this time running alone). Vendor supplied software (FRA Frequency Response Analyzer Software, v3.50) running on a host PC was used to program the equipment and save the readings. The analyzer was programmed to take measurements for the frequency range of $1 \mathrm{~Hz}$ to $100 \mathrm{~Hz}$.

MATLAB was used to plot these results (titled "Frequency Response"). Then, taking the ratio of the DM to $\mathrm{CM}$ gain at each frequency and converting it to decibels, we generated a spectral CMRR curve (titled "Common-Mode Rejection Ratio").

\section{Power Consumption Measurement}

Average power consumed by the circuits, when run from a 3V source, and then from a $5 \mathrm{~V}$ source, was measured. Actual voltage supplied to, and current consumed by, each circuit was measured using a multimeter (Mastercraft Digital Multimeter, CCL 981010178) and the power consumption calculated.

\subsubsection{Results/Analysis}

\section{Input Impedance Measurement}

Figure 3-8 shows results for the input impedance test of the ECG amplifiers. The left plot is for the proposed two-electrode circuit and the right plot for the reference threeelectrode circuit.

The proposed ECG amplifier circuit shows almost flat DM and CM input impedances, indicating that the source load is predominantly resistive. The CM impedance is about $35 \mathrm{~K} \mathrm{Ohm}$ and the DM impedance about $3 \mathrm{M} \mathrm{Ohm}$, almost two orders 

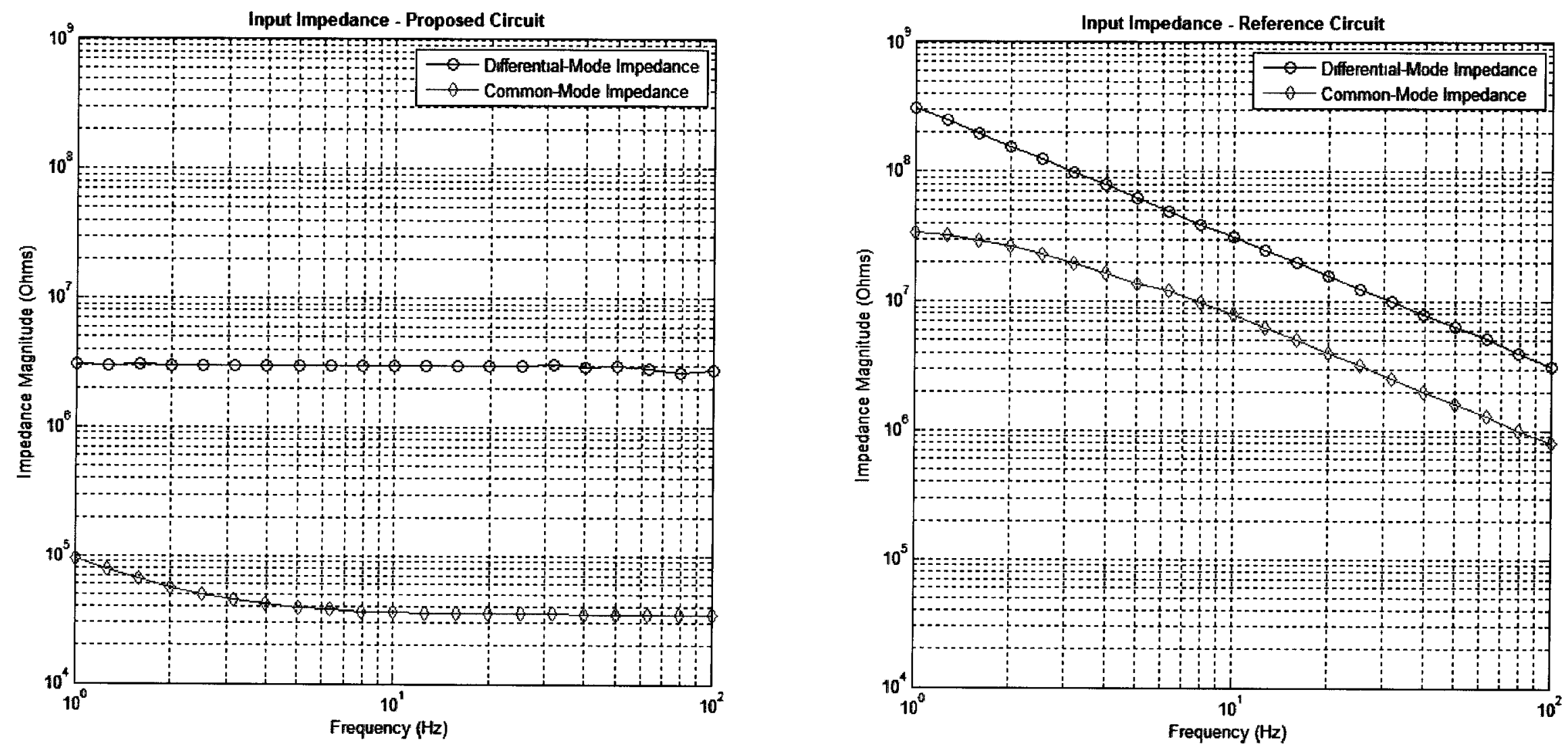

Figure 3-8: Input impedance of the proposed ECG amplifier (left) and reference circuit (right). 
of magnitude larger. This was as expected and is essentially the impedance presented by the CM shunting circuit. In the case of both the DM and CM impedances, the shunt circuit loads overshadow the effect of the input load resistors (i.e. R1 and R2, in Figure 3-3).

The reference circuit, on the other hand, has downwards sloped impedance curves, indicating a mostly capacitive load being presented. It is the high frequency snub capacitors in the input conditioning stage (i.e. $\mathrm{C} 1$ and $\mathrm{C} 2$, in Figure 3-4), that explains this response. To a DM signal the load is the effect of $\mathrm{C} 1$ and $\mathrm{C} 2$ in series (i.e. $500 \mathrm{pF}$ ), and to a CM signal, the load is these capacitors in parallel (i.e. $2000 \mathrm{pF}$ ). This results in the DM and CM impedances differing by a factor of four.

The implications of these observations are that:

- Due to its low CM input impedance, the proposed amplifier will have more CM noise converted to DM noise (as a result of any imbalance between the skin-to-electrode interface impedances of the two electrodes) than had the shunting circuit (comprised of U1A and U1D, in Figure 3-3) not been in place. On the other hand, the circuit ground of the proposed circuit will track the body CM potential more closely and with less lag than if the shunting circuit had not been in place. So implementing the shunting circuit (and choosing best DM and CM impedances) is a design trade-off between reducing power-line interference in the amplifier output and reducing the amount of motion artefact (and possible signal clipping) in the output.

- As there is a larger DM electrode current in the proposed circuit than in the reference circuit, there is a greater DM signal attenuation at the skin-to- 
electrode interfaces of the proposed circuit. This attenuation is DM Signal Attenuation

$$
=\frac{\text { skin_to_electrode interface inpedances }}{\text { DM } \text { input impedance }+ \text { skin_to_electrode interface inpedances }}
$$

The dry electrodes have a combined impedance of about 50K Ohms (from Figure 3-7), so for the proposed circuit,

$$
D M \text { Signal Attenuation }=\frac{50 \mathrm{~K} \Omega}{3 M \Omega+50 \mathrm{~K} \Omega}=1.6 \% \text { or }-0.14 \mathrm{~dB}
$$

This small effect was considered a reasonable design compromise.

- For each electrode, there is a current from the internal body which travels through the skin-to-electrode interface through the ECG electrode input load (the CM load presented by the shunt circuit, in the case of the proposed amplifier) and then through the coupling between circuit ground and earth ground $\left(Z_{G N D}\right.$, in Figure 3-9). The voltage at the first electrode input due to the body's CM potential is therefore:

$$
V_{i n 1}=V_{B o d y C M} \frac{Z_{i n 1}+Z_{G N D}}{Z_{e l 1}+Z_{i n 1}+Z_{G N D}}
$$

Similarly for the second electrode. A DM signal is created as a result of the mismatch of amplifier input impedances $\left(Z_{i n 1}\right.$ and $\left.Z_{i n 2}\right)$ as well as the mismatch of skin-to-electrode impedances $\left(Z_{e l 1}\right.$ and $\left.Z_{e l 2}\right)$. The amount of noise conversion will also depend on the coupling between circuit ground and earth ground. That is,

$$
V_{D M}=V_{B o d y C M} \frac{Z_{i n 1}+Z_{G N D}}{Z_{e l 1}+Z_{i n 1}+Z_{G N D}}-V_{B o d y C M} \frac{Z_{i n 2}+Z_{G N D}}{Z_{e l 2}+Z_{i n 2}+Z_{G N D}}
$$




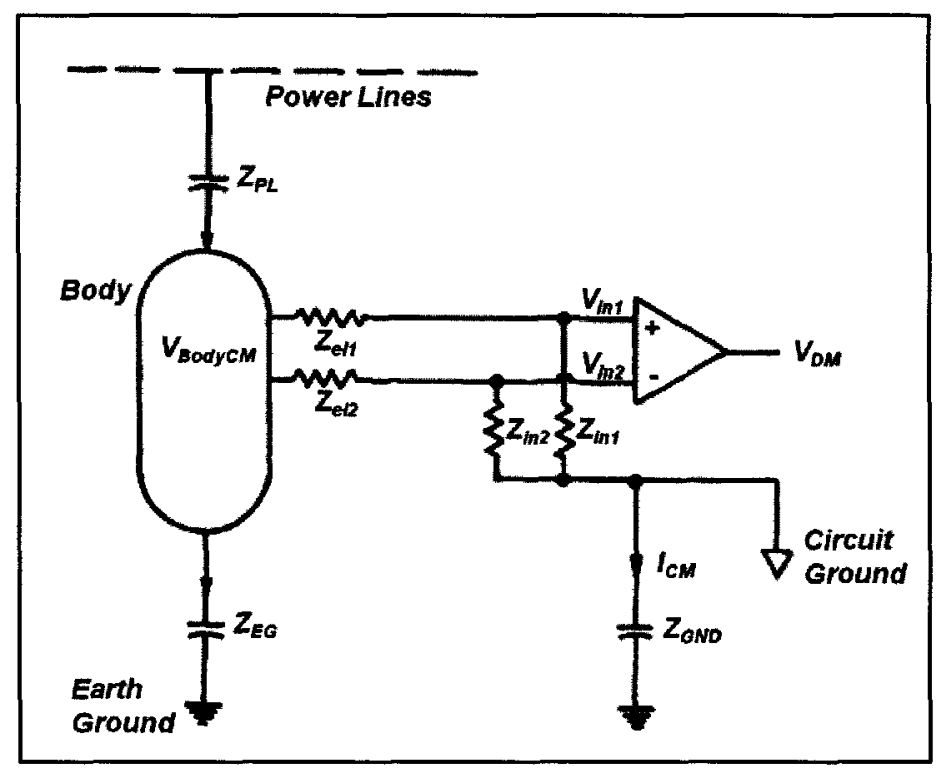

Figure 3-9: Power-line noise model for two-electrode ECG amplifier.

Which, when $Z_{G N D} \gg Z_{e l 1}, Z_{e l 2}$ and $Z_{G N D} \gg Z_{i n}, Z_{i n 2}$,

$$
V_{D M} \cong V_{B o d y C M}\left(\frac{Z_{i n 1}-Z_{i n 2}}{Z_{G N D}}\right) \cong I_{C M}\left(Z_{i n 1}-Z_{i n 2}\right)
$$

If there is no ground coupling (i.e. infinite impedance between circuit and earth grounds), for example, there is no CM electrode current and so no resulting DM noise! In practice there will be some capacitive coupling and so the amount of induced DM noise will be proportional to the electrode impedance imbalance and to the CM electrode current. Ground coupling in this application is expected to be small (this also being borne out by observations in section 3.6.2, where little $60 \mathrm{~Hz}$ signal is visible). It should be noted that if grounded test equipment is used to make measurements during testing of this system, the coupling will suddenly become large (i.e. $Z_{G N D} \approx$ 0) and significant power-line interference would be observed at the amplifier 
output. In conclusion, so long as there is little ground coupling, the electrode impedances contribute little in the conversion of $\mathrm{CM}$ (power-line) noise to DM noise. Furthermore, small and closely matched load impedances (i.e. $\left.Z_{\text {in1 }} \approx Z_{\text {in2 }}\right)$ at the amplifier input impedances can further contribute to lower CM to DM noise conversion.

\section{Frequency Response and Spectral CMRR Measurement}

Frequency response and spectral CMRR (Figure 3-10, top and bottom, respectively), are plotted for the proposed ECG amplifier (left) and for the three-electrode reference circuit (right). Both circuits show an almost flat (within about $3 \mathrm{~dB}$ ) response for frequencies of interest (about $4 \mathrm{~Hz}$ to $40 \mathrm{~Hz}$ ). Common-mode rejection of the proposed amplifier was at or below that of the three-electrode amplifier, but at most about $10 \mathrm{~dB}$ lower.

The low and high frequency gain roll-offs (of both circuits) were attributed to the analog filters in the respective circuits, and were outside the bandwidth of interest. At mid-band, the proposed circuit showed a gain of about 4000 , whereas the reference circuit showed a gain of about 2000 .

The CMRR of the proposed two-electrode circuit ranged, for the frequencies tested, from about $46 \mathrm{~dB}$ to about $58 \mathrm{~dB}$. The reference circuit showed a uniform CMRR of about 56 $\mathrm{dB}$. The crucial frequencies for isolating the QRS complex (for calculation of heart rate) are those around $20 \mathrm{~Hz}$, where the proposed circuit exhibited a CMRR of about $54 \mathrm{~dB}$ (versus about $55 \mathrm{~dB}$ for the reference circuit). As discussed above, the CMRR in this type of application is not as critical as for most ECG amplifier designs because, in the case 

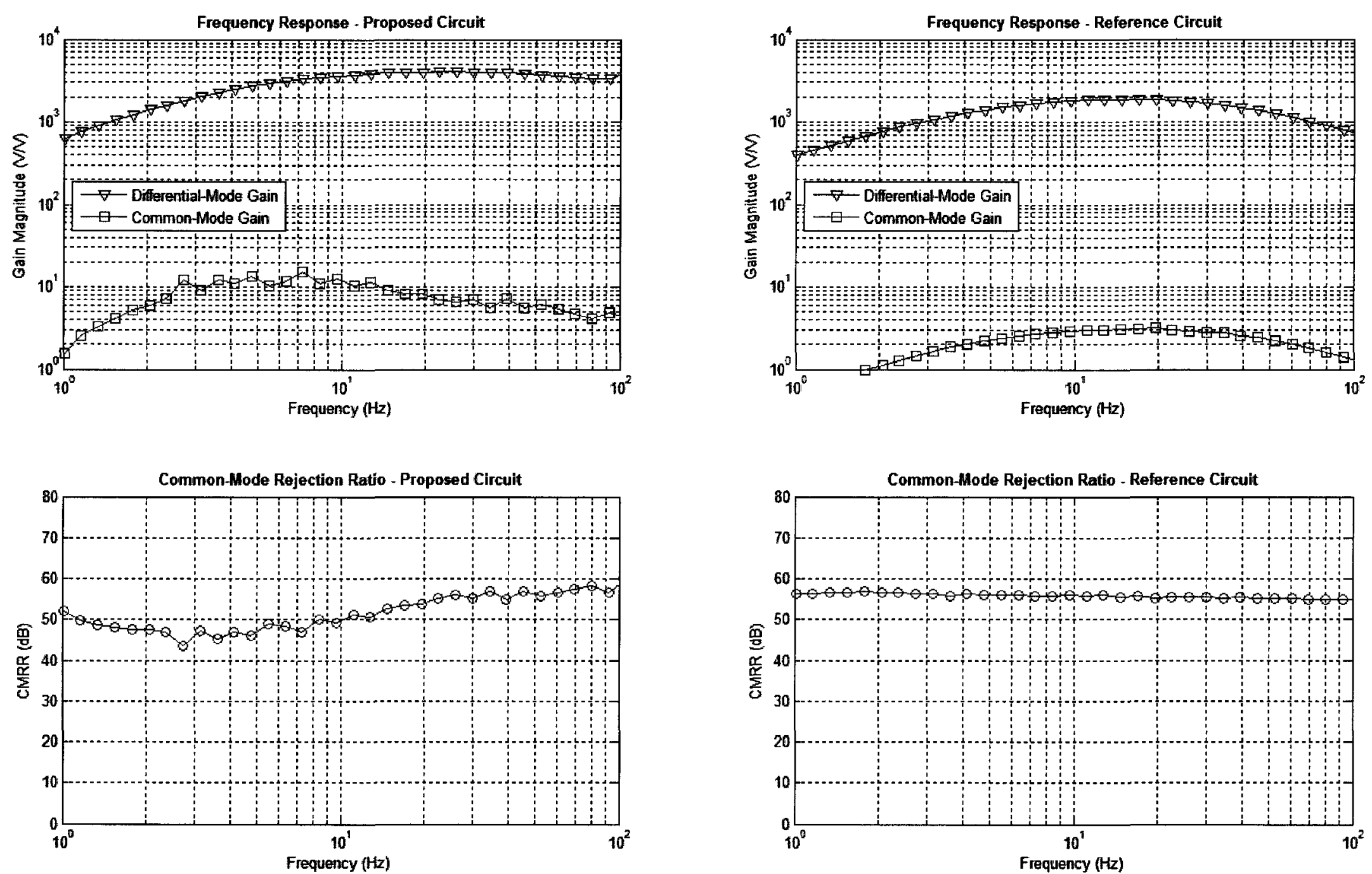

Figure 3-10: Frequency response (top plots) and spectral common-mode rejection ratio (bottom plots), for proposed ECG amplifier (left) and for threeelectrode reference circuit (right). 
of both the two and three electrode amplifiers, circuit ground is forced to track body CM potential (though each uses different mechanism).

\section{Power Consumption Measurement}

Table 3-1 summarizes the power consumed by the proposed two-electrode ECG amplifier (Figure 3-3) and the reference three-electrode ECG amplifier (Figure 3-4). Current consumption, in all cases, was well under the design target of $2 \mathrm{~mA}$.

When supplied by a single 3 Volt battery, both circuits consumed about $1 \mathrm{~mW}$ of power. With a 5 Volt battery pack the power consumption doubled, to about $2 \mathrm{~mW}$. The difference in power consumption between the two circuits is small.

Battery longevity is excellent. The active components used in these ECG circuits are specified for operation down to 2.5 Volts. The load represented by the two-electrode circuit is about $9 \mathrm{~K}$ Ohms $(3.11 \mathrm{~V} / 351 \mathrm{uA})$. Hence, with the battery used in the prototype, a CR2032 lithium coin cell, with this load will discharge down to 2.5 Volts after about

Table 3-1: Average power consumption of proposed and reference ECG amplifier circuits.

\begin{tabular}{|c|c|c|c|}
\hline \multirow{3}{*}{$\begin{array}{c}\text { 3V Battery } \\
\text { (CR2032 Coin Cell) }\end{array}$} & $\begin{array}{c}\text { Actual } \\
\text { Supply Voltage }\end{array}$ & 3.11 Volts & 2.95 Volts \\
\hline & $\begin{array}{c}\text { Current } \\
\text { Consumed }\end{array}$ & 351 uA & 374 uA \\
\hline & $\begin{array}{l}\text { Calculated } \\
\text { Power Usage }\end{array}$ & $1.09 \mathrm{~mW}$ & $1.10 \mathrm{~mW}$ \\
\hline \multirow{3}{*}{$\begin{array}{l}\text { 5V Battery Pack } \\
\text { (4x AA NiCad) }\end{array}$} & $\begin{array}{c}\text { Actual } \\
\text { Supply Voltage }\end{array}$ & 4.93 Volts & 4.92 Volts \\
\hline & $\begin{array}{l}\text { Current } \\
\text { Consumed }\end{array}$ & 388 uA & 439 uA \\
\hline & $\begin{array}{l}\text { Calculated } \\
\text { Power Usage }\end{array}$ & $1.91 \mathrm{~mW}$ & $2.16 \mathrm{~mW}$ \\
\hline
\end{tabular}


25 days (600 hours) of continuous operation (see Appendix A.4 for discharge curves). A battery pack of two AA cells, rated at about 10 times this capacity (2200 mAh is typical for NiMH cells), could be expected to power the circuit for about 8 months of continuous operation.

Though not pursued in this work, cycling the power could potentially achieve significantly lower average current usage, depending on circuit power up time and the required measurement frequency.

\subsection{ECG Monitoring Usage Tests}

\subsubsection{Methods}

\section{Setup}

An ECG monitoring system was assembled, consisting of a rollator with the plate-metal grip electrodes mounted on the handles, the electrodes being wired to the ECG amplifier under test. The output of the amplifier (jumpered to be either the proposed two-electrode circuit or the reference three-electrode circuit) was fed into a differential input channel of a 14-bit ADC module (National Instruments USB-6009). The ECG amplifier was powered by its own battery (a small $3 \mathrm{~V}$ coin cell). Its ground was connected to the ground of the ADC module. The ADC module was then connected via its USB interface (this interface being used for communication of acquired data as well as for obtaining power) to a laptop (a Dell Vostro 1510, running Windows XP and operating from its own rechargeable battery pack). The ADC module and the laptop were strapped onto the rollator. 
Vendor supplied drivers and acquisition software (National Instruments NI-DAQ v7 and LabVIEW SignalExpress 3.0, respectively) were used to measure and record the ECG amplifier output. Metronome software (Weird Metronome, v.1.4) was also run on the laptop to help the subject pace themselves during the experiment. The raw data were stored into files (with no digital signal processing being performed by the acquisition software). MATLAB (MathWorks, v.7.10) was used to process and plot the acquired data.

\section{Procedure}

Four healthy volunteers were recruited. The purpose of, and their involvement in, the research was explained and we obtained their written consent. This research was reviewed and approved by the Carleton University Research Ethics Board.

The tests consisted of recording 60 seconds of ECG data, digitized with 14-bits of amplitude resolution and 1000 samples per second of temporal resolution. The subject was explained the protocol and then asked to start by doing one practice run.

The first test conducted consisted of:

a) Standing in place holding onto the rollator handles "with a normal and comfortable grip", for about 15 seconds.

b) Then walking, at a slow pace, pushing the rollator (about one step per two seconds or 30 steps per minute) for about 15 seconds.

c) Then stop walking, and stand in place again, for about 10 seconds. 
d) Then, finally, to stay standing, stationary, but now gripping onto the handles as tightly as possible, until the recording ended (typically, another 10 or 15 seconds).

The second test was identical to the first one, but using the three-electrode reference circuit instead of the two-electrode proposed circuit. The subject was asked to place their thumbs on the "thumb" (drive) electrodes while gripping the handles.

Data from these recordings were then imported into MATLAB and plotted.

A first set of plots consisted of side-by-side representative slices of each part the experiment, for each of the subjects, along with their calculated power spectrum density (PSD) curves. Processing of the ECG data included:

1) Dividing the measured voltages by the amplifier's gain, so as to show inputreferred signal amplitudes on the plots (this compensates for the slight differences in gain between the amplifiers).

2) Calculating the PSD for the slice. This was using a Welch estimating method with a one second hamming window.

These plots show 5,10 and 5 second slices of activity, respectively, from the regions of each recording where standing (at rest), walking (light activity), and tight gripping (strong activity), were happening.

An additional plot, of an entire 60 second recording (for both the proposed and the reference circuits) from one of the subjects, was then plotted to better visualize the character of the baseline wander (motion artefact) and the high-frequency (EMG) noise. Two additional curves were included in this plot, consisting of these above two curves digitally bandpassed with a high order $4 \mathrm{~Hz}$ to $40 \mathrm{~Hz}$ linear-phase zero-time-lag filter. 
The filtering was achieved using MATLAB's "filtfilt" function, used twice: once for the low-pass filtering and once for the high-pass filtering (see Table 3-2 for parameters used).

Table 3-2: Filter details for attenuation of EMG noise (low-pass) and motion artefact (high-pass).

\begin{tabular}{||l|l|c|c|}
\hline \multicolumn{2}{|l|}{ Filter } & & \\
\hline FIR filter type & Fp - frequency at the edge of the passband $(\mathbf{H z})$ & 40 & 4 \\
\hline \multirow{4}{*}{$\begin{array}{l}\text { Filter } \\
\text { response } \\
\text { parameter }\end{array}$} & Fst - frequency at the beginning of the stopband (Hz) & 60 & 2 \\
\cline { 2 - 5 } & Ap - passband ripple in dB & 1 & 1 \\
\cline { 2 - 5 } & Ast - stopband attenuation in dB & 30 & 30 \\
\hline
\end{tabular}

\subsubsection{Results/Analysis}

\section{ECG while Standing}

The overall amounts of noise in the ECG signal varied by subject (see Figure 3-11 and Figure 3-12). This was attributed primarily to differences in their skin characteristics (amount of sweating, sweat mineral contents, thickness of epidermis, etc.), hand-grip contact area, nominal grip strength and cardiovascular variance among individuals.

The proposed circuit, for all individuals, had noticeably more noise than the recordings with the reference circuit. The PSD curves indicate that this noise is attributable mostly to energy from signal components above $25 \mathrm{~Hz}$. For each test subject, the region of the PSD curve below $25 \mathrm{~Hz}$ is similar between circuits.

The better noise immunity of the three-electrode circuit was attributed to the reference electrode's feedback loop which, with a gain of 100 , creates $-40 \mathrm{~dB}$ of broadband common-mode rejection. In spite of the higher noise level in the proposed circuit output, the ECG signal was, indeed, clearly discernible, with an easily identifiable 

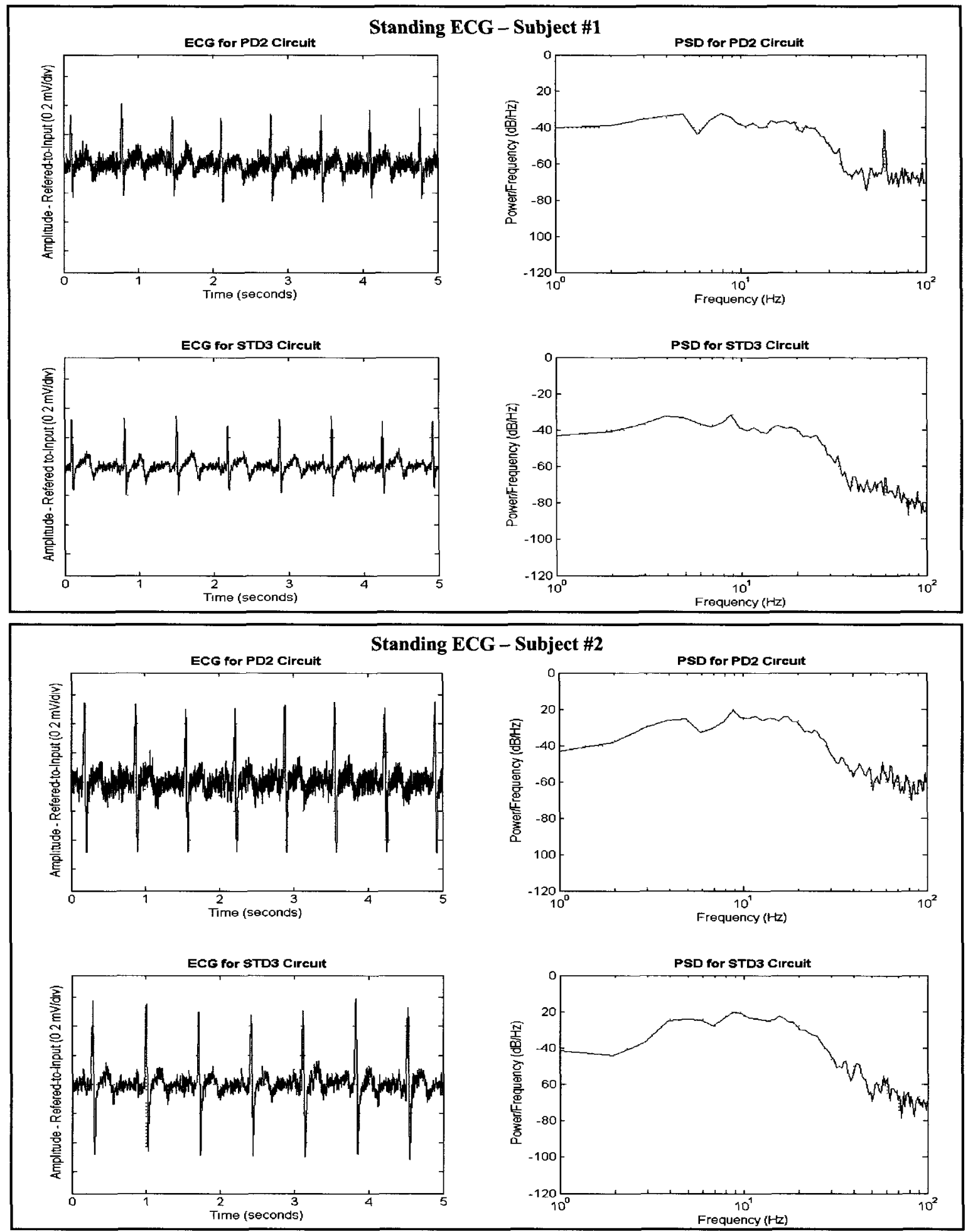

Figure 3-11: Representative samples of ECG for subject \#1 and \#2, while standing still. Top plots for each subject are for the proposed two-electrode ECG amplifier. The bottom plots are for the standard three-electrode amplifier. 

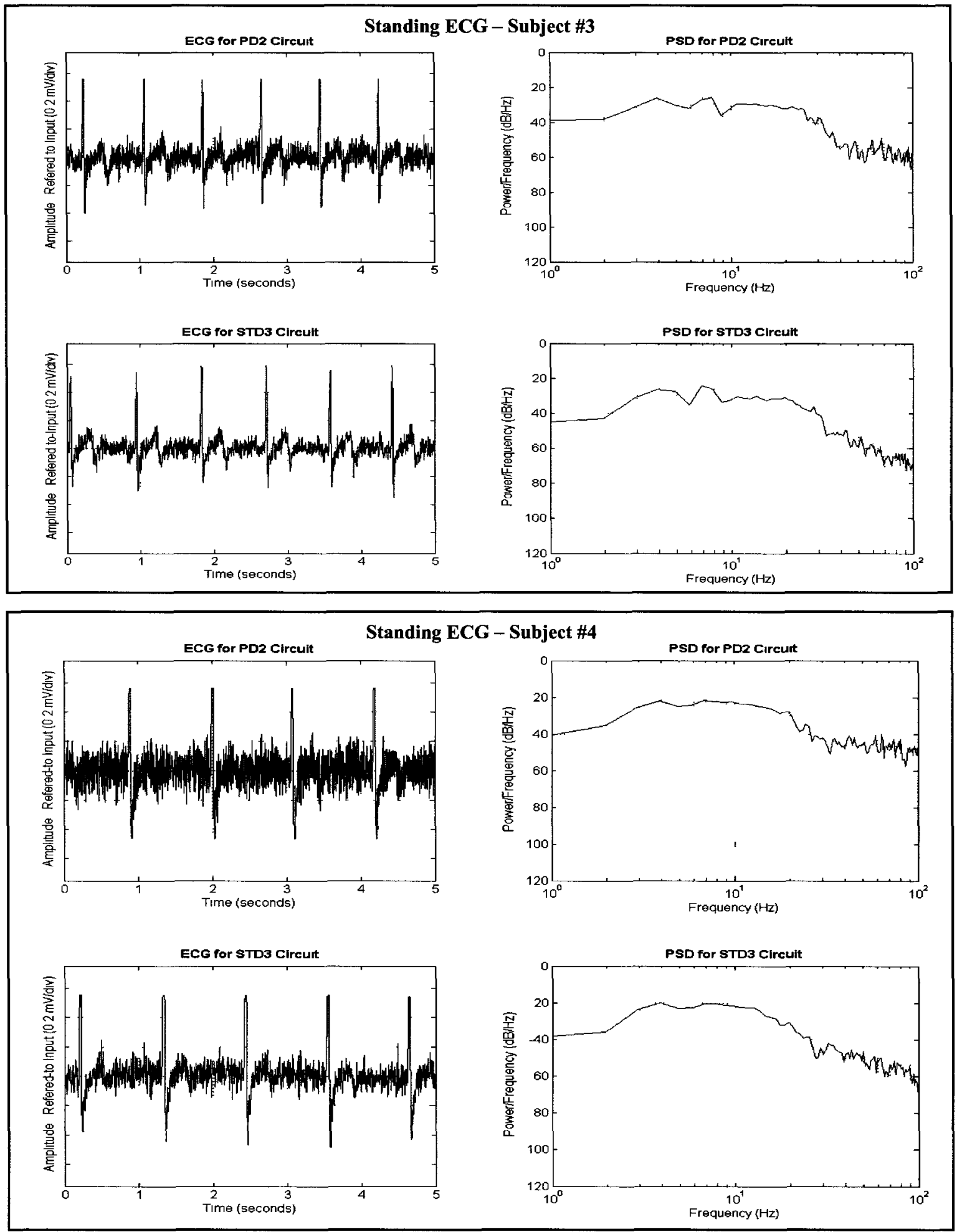

Figure 3-12: Representative samples of ECG for subject \#3 and \#4, while standing still. Top plots for each subject are for the proposed two-electrode ECG amplifier. The bottom plots are for the standard three-electrode amplifier. 
R-peak. The noise levels, in none of the recordings of standing ECG, confounded visual identification of the heart beat.

It was noticed, when processing the data, that some of the signals from the proposed circuit (in particular, for subject \#4), had a few clipped R-peaks. This was likely due to the gain of this amplifier being about 3000 , resulting in a signal range of about $3 \mathrm{~V}$ (exactly the supply voltage!). Simply adjusting the gain resistors in one of the amplifying stages to obtain a gain of about 2000 would likely have avoided saturation. The main effect of this clipping is that, where it does occur, the signal-to-noise ratio (SNR) of the affected signals would be underestimated, due to smaller R-wave peaks. (SNR can be visually assessed by looking at the ratio of the R-wave amplitude to the mean noise level during the T-to-P interval). As this occasional clipping did not have a large effect on the analysis being performed, we chose not to repeat the experiments.

Both circuits showed an excellent ability to manage noise. The noise, in these simple experiments, did not overwhelm the ECG signal nor did it cause the signal to be offset sufficiently to cause saturation. We conclude that, based on these preliminary results, during times when the user is not walking, reliable extraction of a heart beat using digital methods is likely feasible.

\section{ECG while Walking}

The amount of noise was greater in all of the walking ECG tests than the corresponding standing ECG tests (see Figure 3-13 and Figure 3-14). There is evidence of greater muscle activity, evidenced by high-amplitude high-frequency EMG noise. One can also observe some low frequency baseline wander resulting from balancing action, from 

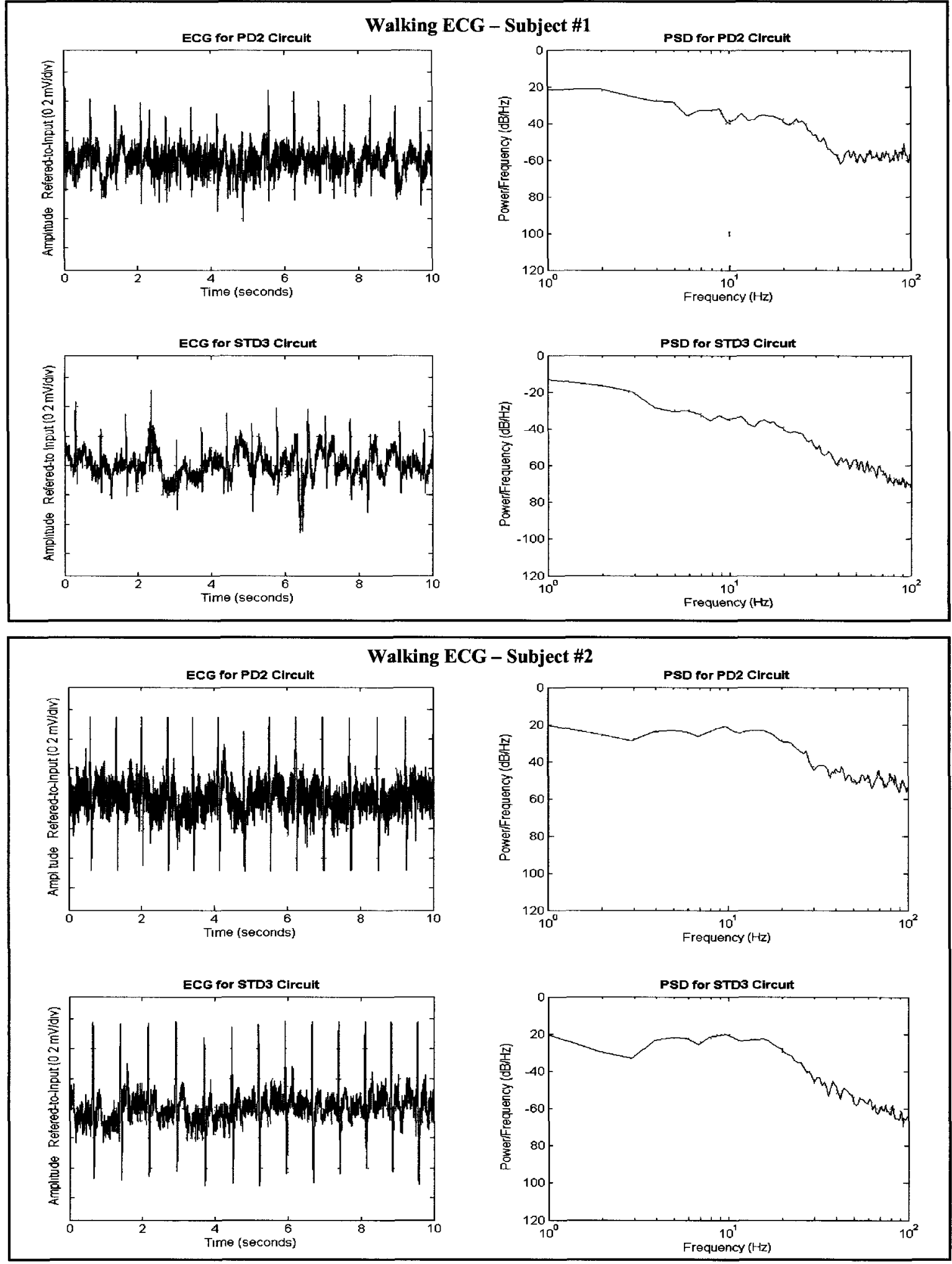

Figure 3-13: Representative samples of ECG for subject \#1 and \#2, during a slow walk. Top plots for each subject are for the proposed two-electrode ECG amplifier. The bottom plots are for the standard three-electrode amplifier. 

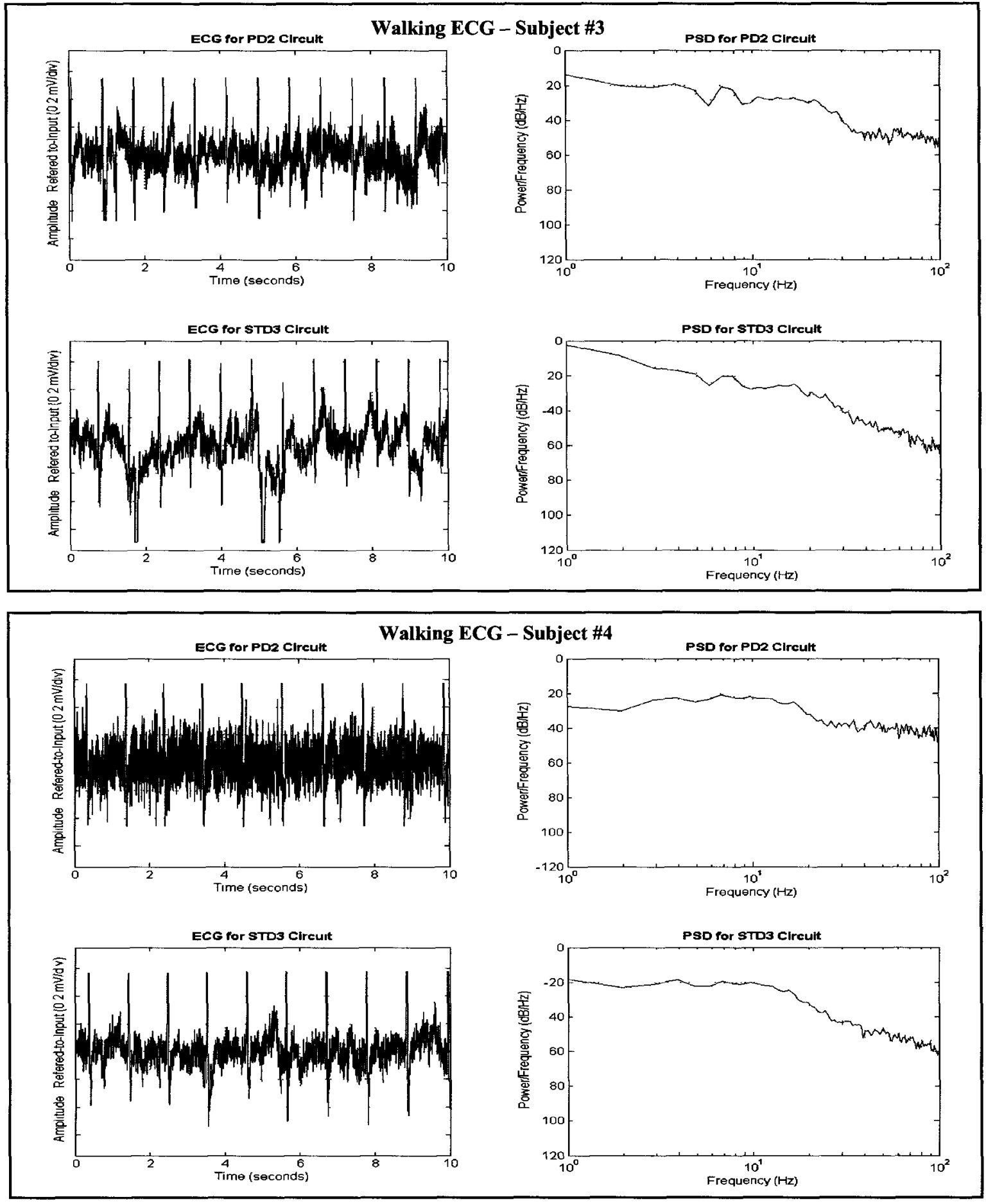

Figure 3-14: Representative samples of ECG for subject \#3 and \#4, during a slow walk. Top plots for each subject are for the proposed two-electrode ECG amplifier. The bottom plots are for the standard three-electrode amplifier. 
one's grip on the electrodes, as each step is taken. Though the R-waves are clearly discernable, the noise levels in some of these recordings (e.g. for subject \#1) at times confounded visual identification of the heart beat. These are unprocessed signals, and so further study would be warranted to see if further processing can manage, or at least identify and tag, the confounding noise. The section on "Signal Quality", below, begins such an analysis.

If we look at any one subject's tests, there is visually more high frequency noise evident in the proposed circuit's output. The PSDs confirm this by, once again, showing differences in power content only for frequencies above about $25 \mathrm{~Hz}$. This suggests that processing the data with a low-pass filter could result in significant quality improvements.

Low-frequency noise is also visually apparent, the signal's baseline moving up and down every half to four seconds. This suggests that a high-pass filter to attenuate this outof-band low-frequency noise could be quite effective. This could easily be performed digitally, with little or no added distortion.

\section{ECG while Gripping}

As this was the last part of each user test, only two of the four subject's tests yielded sufficient "tight grip" data for analysis (Figure 3-15). Data collection for the other two subjects ended at less than 2 seconds, an insufficient amount of "tight grip" data for analysis. Here we notice that high frequency noise often overwhelms the ECG signal. Comparing the PSDs to the standing tests for subjects 1 and 2, respectively (Figure 3-11), we see that the "extra" signal is predominantly $25 \mathrm{~Hz}$ and above. 

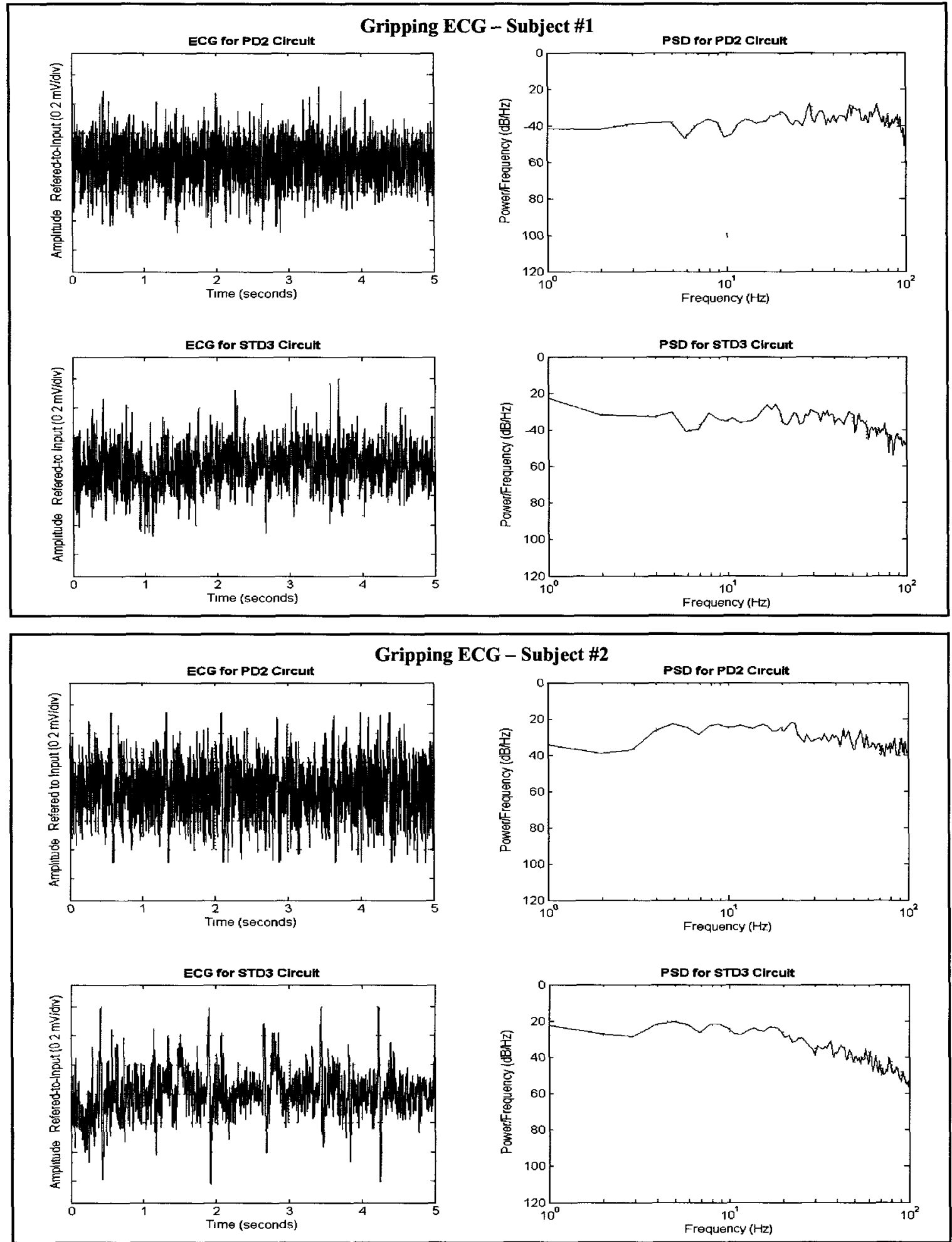

Figure 3-15: Representative samples of ECG for subject \#1 and \#2, while gripping tightly on the electrodes. Top plots for each subject are for the proposed two-electrode ECG amplifier. The bottom plots are for the standard three-electrode amplifier. 
A visual assessment suggests that extraction of a heart beat from this signal may be difficult, unreliable or even impossible. Further study in processing of these signals would be warranted if monitoring during such "intense" periods is desirable.

\section{Signal Quality}

As a quick test to assess how well the motion artefact and EMG noise could be managed though digital post-processing, bandpass filtering was performed on the data of one of the test subjects.

Figure 3-16 plots the raw and digitally processed signals, for the proposed circuit and the reference circuits respectively, in traces 1 and 3 (counting from top to bottom). Regions of ECG include: (a) standing, (b) walking and (c) tightly gripping the electrodes. Just below these regions is the corresponding processed (bandpass filtered) signal. Although the proposed two-electrode circuit (top curve) showed more overall noise than a standard three-electrode system (third curve), once bandlimited to the frequencies of interest using a digital filter ( 4 to $40-\mathrm{Hz}$ ), both resulted in equally useful heart beat markers (second and fourth curves).

The bandpass range and order of the filter, for this preliminary study, were arrived at empirically. A more rigorous assessment of filtering needs could likely yield higher quality results and a better understanding of processing needs (linear and/or algorithmic).

Based on the limited results presented here, for periods of standing and walking a robust heart beat signal appears to be achievable by simple bandpass filtering. Identification of heart rate for periods of intense muscle use may also be possible but 


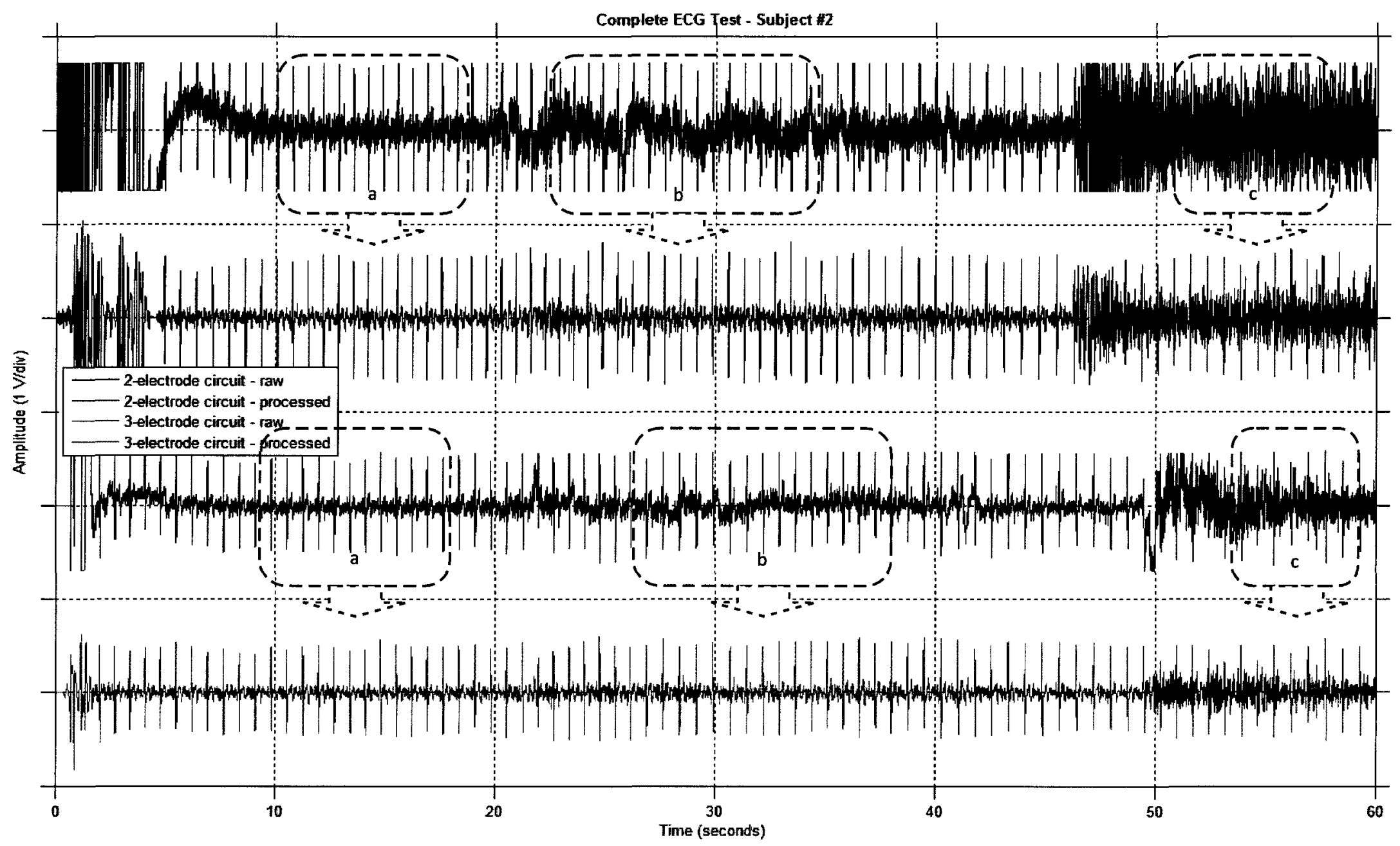

Figure 3-16: 60-second ECG recordings for one subject, including a period of (a) standing still, (b) walking slowly and (c) griping the electrodes tightly. 
would require more extensive processing of the data.

\subsection{Conclusions}

Adding ECG monitoring to a rollator had some challenges in addition to those of a typical clinical ECG application. Those which most impacted the design of the ECG amplifier circuit were:

1. During normal use, the user continually changes the tightness of their grip and the weight placed on the handle grips. This has an effect of creating large changes in the DC offset at the amplifier's electrode inputs. Such large drifts in the ECG baseline could easily cause the amplifier circuit to saturate. Where changes are not sufficient to cause saturation but the baseline changes occur quickly, the higher frequency components of this noise may confound ECG and HR processing algorithms.

2. The user, frequently being in motion, will have a dynamically changing coupling to both ground and the power lines, changing both the amplitude and phase of power-line interference.

3. Not having a third (reference) electrode to directly control the CM voltage of the subject's body, an alternate strategy for managing the effects of this significant noise source is needed.

The proposed ECG amplifier addresses these needs by:

1) A careful choice of a high-pass filter at the input stage of the amplifier attenuated the low $(\ll<\mathrm{Hz})$ noise considerably, avoiding saturation of the 
differential amplifier inputs and of the later amplifying stages. This even with a very low supply voltage (power supply rails of $+/-1.5 \mathrm{~V}$ ).

2) A CM shunt circuit at the amplifier input helped make the body CM potential and the amplifier circuit's ground track each other.

Testing a prototype system which incorporated the designed ECG amplifier yielded the following general observations:

a) The quality of the ECG signal differed considerably between individuals. Both amplitude and noise content varied.

b) With some simple digital signal processing, the ECG output from the twoelectrode amplifier showed promising results. For the data processed (one subject only), heartbeat information was clearly isolated during standing (subject simply holding the rollator handles) and while walking.

c) During higher activity levels the ECG was visible, but further study would be needed to establish how reliably it can be extracted (across users and types of activities).

Further follow up study yielding much benefit could include:

$>$ A larger study to confirm the results of this work and to better evaluate the variance in ECG extraction quality across a population.

$>$ Comparison of different types of dry electrodes (such as rubber electrodes) to evaluate their performance for ECG monitoring. Bench-top characterization and standardized usage tests would be appropriate.

Field testing the circuit against a clinical grade portable ECG monitor, such as a Holter. Also creating a printed circuit board version of this circuit would 
have the benefit of miniaturization, and would likely add performance while making the circuit reliable enough for field testing.

$>$ Finally, analysis of results of usage tests, with the development of digital signal processing approaches to maximize heartbeat identification during normal rollator use. 


\section{Chapter 4: Speed/Distance Monitoring}

\subsection{Introduction}

Adding non-obtrusive sensing and recording capabilities to a rollator offers potential benefits for healthcare and manufacturing design (Chan \& Green, 2008). One of the modalities of the Smart Rollator system is the measurement of distance travelled as well as its derivative, speed of travel. Data, once collected, can be used, for example, to provide patients and their healthcare provider usage statistics for the device, to aid in compliance to a usage regimen. Data could also have value for rollator manufacturers, to better understand how their devices are being used in the field (common usage patterns, frequency of falls, etc.). Combining these data with other sensed information (e.g. incline of the device, user's heart rate and ambient temperature) could add further value. For example, speed as a person goes up or down slopes and heart rate in terms of effort and speed.
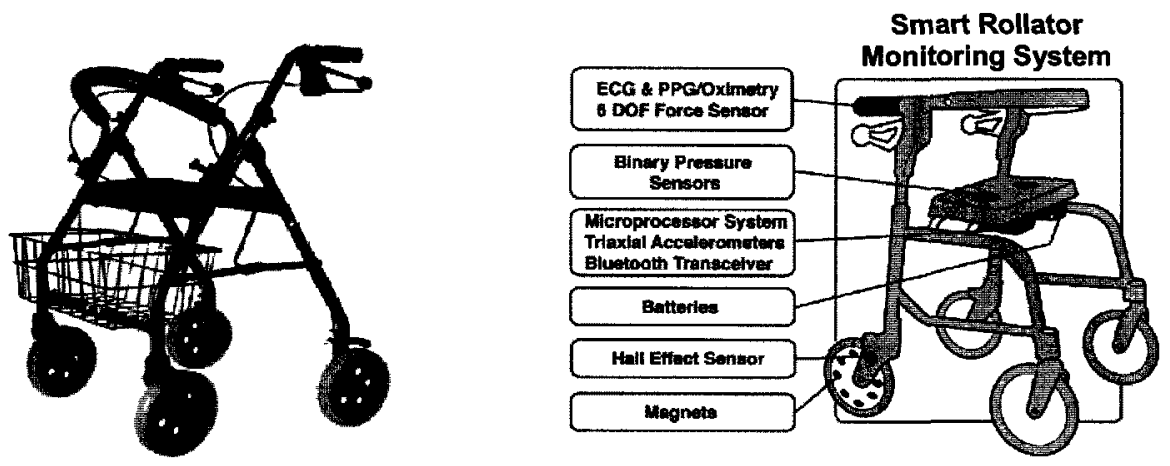

Figure 4-1: Example of a commercially available rollator (left) and the Smart Rollator monitoring system being developed at Carleton University (right). 
A previous design of the Smart Rollator provided a simple measurement of absolute travel (Agnello \& Earl, 2008). By attaching magnets near the periphery on one of the device's wheels and attaching a magnetıc sensor onto the fork of the wheel, the resultıng signal was used as an indication of movement of the device (Figure 4-2). As the magnets pass beneath the sensor, pulses are generated. The pulse count is multiplied by the arc distance subtended by the inter-magnet angle to obtain a travel distance.

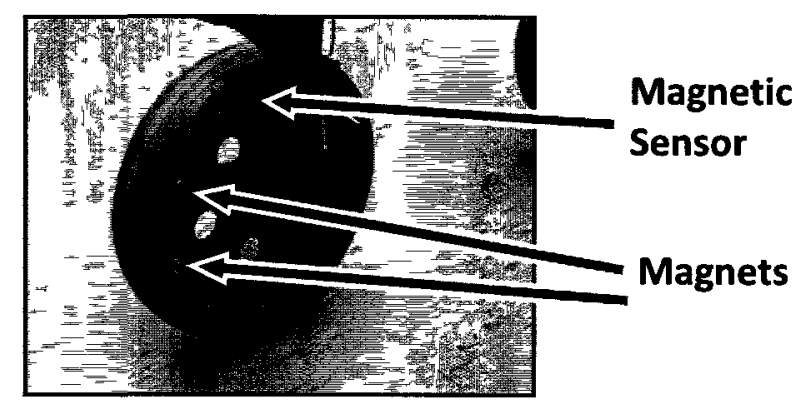

Figure 4-2: First generation distance/speed sensing on the SmartRollator system.

This design approach, of using permanent magnets and a magnetıc reed switch, has the advantage of being quite resistant to mechanical variances such as wheel wobble and rollator vibration (experienced, for example, when moving over a rough surface). Also, it is a simple design, inexpensıve to implement and can also scale up to higher resolution by including more (equally spaced) magnets along the periphery of the wheel. Finally, the output, being digital, interfaces efficiently into electronic counters as well as the counting modules included in most modern microcontrollers.

\subsubsection{Focus of Research}

This approach, however, also has some possibilities for improvement. If augmented to record both the magnitude and direction of the wheel's rotation, more detailed motion information is available for analysis. 
Also, the previous design is susceptible to false distance tallying when hovering around a sensor trigger point. The magnetic sensor, in this case, toggles on and off repeatedly while the user is remaining essentially stationary, resulting in a position drift artefact. This occurs when the magnetic field at the sensor repeatedly increases past and decreases below the sensor's threshold. It is because the sensor system indicates that the wheel is moving, but not the direction of the wheel's rotation, that this artefact occurs.

A proposed improved design is presented and evaluated in this chapter. A second sensor is added to the system in such a way as to generate a two-bit Gray code sequence suitable for up/down counting by a quadrature decoder. The result is the measurement of net travel distance. The sensor pulses, as with the previous design, can also be individually counted to obtain an absolute travel distance.

It should be noted that sensing the wheel's rotation does not indicate actual direction the rollator is moving in, but rather, only if it is going forward or backwards. If the user, for example, steers the Rollator to the left or to the right while still going forward, this will be accumulated as "positive" counts. So what is being measured is movement rather than location change, and its calculated derivative, speed rather than velocity.

\subsubsection{Related Works}

An electronically equipped rollator being developed at the University of Toronto includes an optical encoding technique to monitor movement of the device (Tung, et al., 2007). Alternating strips of black and white are painted onto the side of the wheel, with a dual optical reader sensing wheel rotation and direction. Though this is a simple design and perhaps suitable for a controlled environment, it could suffer from interference when 
used outdoors or in places with varying levels of ambient lighting. It may also experience missed readings should the strips become soiled.

Several other designs of wheel motion sensing systems have also been discussed in the literature, for monitoring wheelchair use. Some of these use a magnet and reed switch design (Cooper, et al., 2002) (Sonenblum, Sprigle, Harris, \& Maurer, 2008) (Tolerico, et al., 2007). These, however, are all single pulse counting systems and so would still need their designs augmented to reliably provide directional (i.e. up-down) counting capability and have the advantages of the design being presented in this chapter.

A robotic system, designed for the U.S. Department of Defence, uses a magnetic ring attached to a wheel and two Hall effect sensors. It is discussed in the context of a larger autonomous vehicle design (Everett, Pastore, \& Bonner, 1999). Such magnetic rings, one example having 320 magnetic polarity changes per rotation (RLS Merilna Tehnika, 2009), are available commercially and provide a quadrature encoded output. As this would avoid the need to mount individual magnets on a wheel, this design is an alternative to the one being presented in this chapter, particularly if higher resolution is required. However, this is a significantly more costly approach and would still require the empirical design method being outlined here. Hall effect sensors for magnetic sensing may also be suitable for our design (discussed further in Section 4.2.3), but again, at a higher cost due to more complex interfacing requirements. 


\subsection{System Design}

\subsubsection{Rotary Quadrature Encoders}

The rolling mechanism of a rollator typically consists of two front wheels, which can swivel to facilitate turning, and two back wheels, fixed, which roll in the forward/backward direction only (relative to the rollator orientation). In the design presented here, we measure the rotation of one of the back wheels in order to calculate distance moved.

A popular and practical method of measuring a wheel's rotation is by using a rotary encoder. Rotary encoders are inexpensive and come in a wide range of resolutions and packages (Figure 4-3a is an example). Rotary encoders create two output signals, these being out of phase square waves whose period and phase depend on angular speed and direction, respectively.

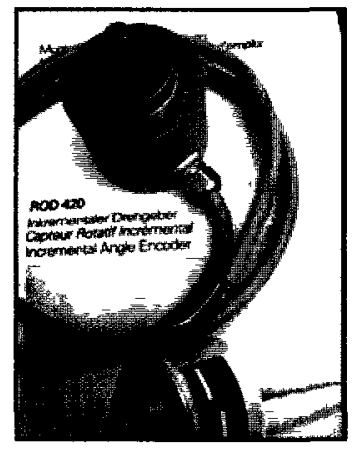

(a)

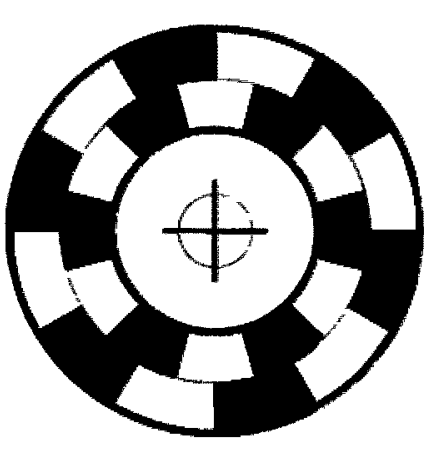

(b)

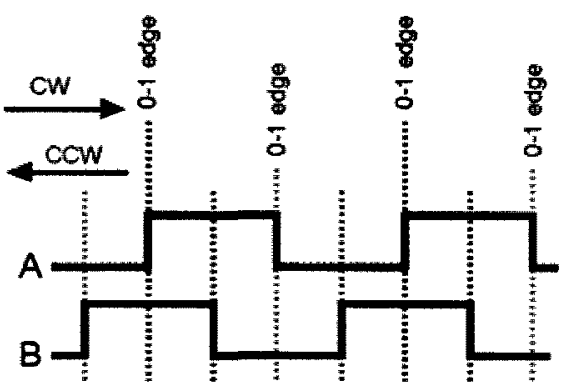

(c)

Figure 4-3: Rotary encoders (a) measure rotation by sensing edges and levels of two out-of-phase slotted tracks (b). Angular displacement is measured by counting transitions, with the direction of each sensed displacement being indicated by the polarity of the other channel (c).

Optical rotary encoders typically contain a disc, having a pattern of slots or dark areas, mounted on an axle (Figure 4-3b). The system is enclosed in a light-proof and protective housing. Two optical sensors sense the pattern as the disc is rotated. The 
quadrature signal, a two-bit wide digital sequence is generated, these channels normally being labelled as "A" and "B" (Figure 4-3c).

A quadrature signal is processed by a decoder/counter, which differentiates rotary movement and direction. Counter modules of many microcontrollers now have (in addition to simple pulse counting) integrated quadrature processing, automatically performing the up or down counting in hardware in response to shaft rotation, with minimal software overhead.

Rotary encoders, typically, are physically coupled directly to a rotating axle, or else indirectly through a gear or pulley. The Smart Rollator, based on a Nexus III rollator (Dana Douglas Inc.), however, has wheels with fixed rather than rotating axles. As our preference is in retrofitting existing rollators rather than making modifications to the design of the rollator, another way of mechanically coupling an encoder would be needed. It would be possible to couple to the rotating wheel using gears or belts, but it was felt that this would increase cost and diminish the reliability of the system, so other sensing methods were considered.

\subsubsection{Magnetic Encoding}

Recognizing that a quadrature signal is a good method for coding directional displacement, we explored the idea of obtaining a quadrature signal using magnets and magnetic sensors, as this worked well for the previous one-bit design. Our aim was to provide the ease of interfacing and processing of an optical rotary shaft encoder signal, while still retaining the simplicity and robustness of the previously used magnetic design. 
One approach would be by having two identical "tracks" of rotating magnets, the tracks being at different distances from the wheel's axle and slightly out of phase. Then with a magnetic sensor above each track, one could generate a quadrature signal similar to that of an optical encoder. This approach, however, would require twice the number of magnets than the previous design and the tracks would need to be sufficiently separated so that the fields of one track do not interfere with those of the other.

Other approaches to building the encoder were considered. By observing that the tracks for signals A and B in Figure 4-3b had the same pattern with only a phase difference, we then considered using only one track of magnets, but with two magnetic sensors to read the magnets' fields, offset by some fraction of the inter-magnet distance.

However, due to the complexity of calculation and the lack of availability of detailed magnet and sensor engineering specifications, the choice and placement of components in such systems cannot be accurately determined using analytical methods (Hamlin Inc., 2007) (LaBarge \& Gutierrez-Miravete, 2008). As such, characteristics of these key components need to be ascertained empirically prior to the system being designed and validated. The bulk of the efforts in this study were therefore in characterizing the components within a proposed system.

\subsubsection{Selection of a Magnetic Sensor}

Three popular types of magnetic sensors are the sense coil, the Hall effect device and the reed switch. Some of the advantages and limitation of using these technologies, with respect to this application, are summarized in Table 4-1. 
The signal generated by a sense coil, being proportional to the rate of change of the magnetic field passing through it, presents design challenges in reliably accommodating a wide range of pulse amplitudes. And as the rollator is frequently operated at very low speeds, the sense coil was considered unsuitable for this application.

Table 4-1: Advantages and limitations of some popular magnetic sensors.

\begin{tabular}{|c|l|l|}
\hline & & \\
\hline Sense coil & - Reliable & $\begin{array}{l}\text { - Low sensitivity at low frequencies } \\
\text { - Complex interface }\end{array}$ \\
\hline $\begin{array}{c}\text { Hall effect } \\
\text { device }\end{array}$ & $\begin{array}{l}\text { - Small sensor } \\
\text { - Reliable }\end{array}$ & $\begin{array}{l}\text { - Significant power requirements } \\
\text { - Complex interface }\end{array}$ \\
\hline Reed switch & $\begin{array}{l}\text { - Simple to interface to } \\
\text { - Inexpensive } \\
\text { - Rugged mounting package }\end{array}$ & $\begin{array}{l}\text { - Contact wear and failure } \\
\text { - Mechanical contact bounce }\end{array}$ \\
\hline
\end{tabular}

Using a Hall effect device is a viable option, so long as it is cycled, to reduce overall power consumption. These devices typically take $3 \mathrm{~mA}$ to $10 \mathrm{~mA}$ during continuous operation (Allegro Microsystems Inc., 2010) (Asahi Kasei EMD Corporation, 2009) (Honeywell International, 2005), too high if the goal is to run off a small battery for days or weeks. An interesting option is the Hall effect "switch", an integrated circuit which also includes interface electronics and which is now available with fast power-on time and in low voltage versions (Allegro Microsystems Inc., 2010) (ROHM Semiconductor, 2010). Hall effect components, if used outside of a laboratory, would need to be packaged and mounted for reliable operation.

A reed switch, due to the ease of electrically interfacing the sensor to a microcontroller, low power requirements and low cost, was chosen for this design. The component used in our design comes as a hermetically sealed threaded assembly, 
providing easy installation (see photo in top right part of Figure 4-8a, and details in Appendix A.6 ).

\subsubsection{Selection of Magnets}

Magnets are available in a variety of sizes, shapes and strengths. For compactness and to maximize flexibility in sensor-to-magnet spacing, we chose to use powerful rare earth magnets for this application (Radial Magnet Inc., 2010). We tested our design with two sizes of magnets, to assess the practical impact of dimension and strength on performance (see Appendix A.5 for specifications).

The width of the magnets, relative to magnet spacing, is also expected to have an impact on the system's behaviour. Along the circular track of magnets, regions of "high amplitude" (i.e. where the magnetic field is above the magnetic sensor switch-on threshold) and "low amplitude" (i.e. below the switch-off threshold) of about the same arc length would create a duty cycle of around $50 \%$ and yield a good immunity to pulse jitter. This will be discussed in more detail in section 4.2.7.

\subsubsection{Field Potential and Orientation of Magnets}

To get an idea of the field surrounding the track of magnets and to help predict the response of the sensors, some software simulations were run. This simulation was performed with COMSOL v3.5. The goal of the simulations was to get an idea of the shape of the field surrounding the wheel, and in particular to see how this shape depended on the magnet orientation pattern. As this was intended to be a comparative analysis, reasonable values were used for magnet strength and dimensions. If an exact quantitative analysis were required and if the parameters for the magnets were known (e.g. separation, 
size, material and magnetization) the simulation could be rerun to obtain numerically accurate results.

Reed switches operate optimally when the North-South (N-S) axis of an acting magnet is in-line (or nearly in-line) with the contacts of the switch (Hamlin Inc., 2008) (LaBarge \& Gutierrez-Miravete, 2008). So, when orienting the sensors to be perpendicular to the plane of the wheel (which was the case with the sensors used in this design), the axis of the magnets should also be perpendicular to the wheel's plane. However, the magnets in the track could either all have identical N-S orientations, or each magnet could have the reverse N-S orientation from its adjacent magnets (see Figure 4-7a). The latter case, of course, would require an even number of magnets be installed on the wheel. By modeling these two orientation patterns, we can observe the effect of the rotating track of magnets on field intensity at a fixed sensor location.

Therefore, two configurations were simulated: an alternating magnet orientation and a unidirectional orientation (shown in Figure 4-4, top and bottom images, respectively). In both cases, the N-S axes were perpendicular to the plane of the wheel. In the former case adjacent magnets had their N-S poles in opposite directions and in the latter case the magnets all had their $\mathrm{N}$ poles facing the same direction.

These images give the magnetic flux density vector (arrows) plotted as a function of radial distance (i.e. distance along the arc of the track of magnets) for the $\mathrm{x}$-axis, and as a function of distance from the plane of the wheel for the y-axis. The contour lines represent locations of equipotential magnetic strength.

A typical trajectory of the magnetic sensors (relative to the wheel's position) is indicated by the dashed lines in these plots. For this simple simulation, this path is along 


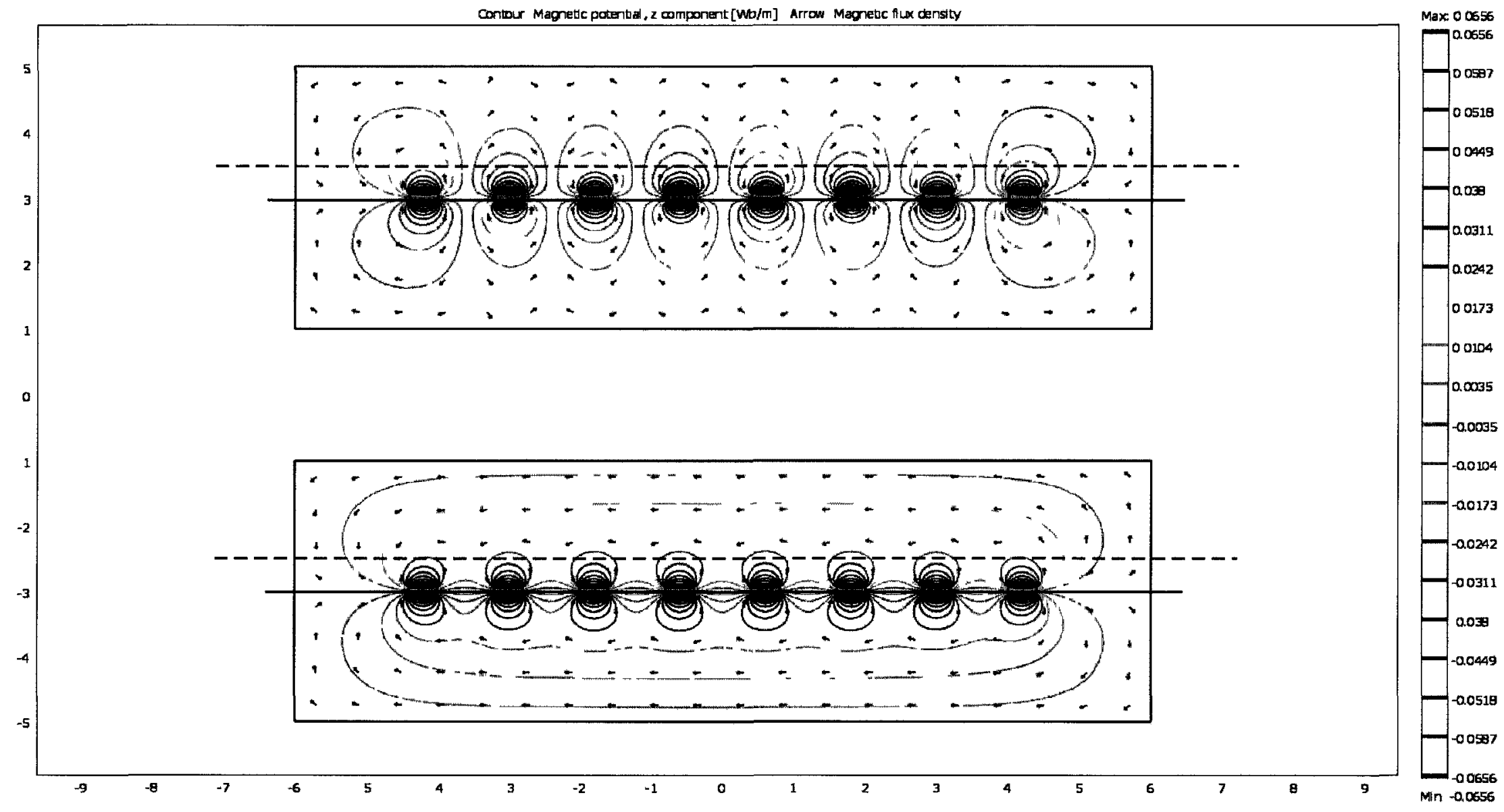

Figure 4-4; Spatial distribution of magnetic potential for cases in which magnets are arranged in with alternating polarity (top) and unidirectional polarity (bottom). The solid line indicates the plane of the wheel. Dashed lines show possible trajectories of the magnetic sensors. The y-axis in this plot represents distance from the plane of the wheel. The $x$-axis represents radial distance (inter-magnet separation along the circumference of the track of magnets.) 

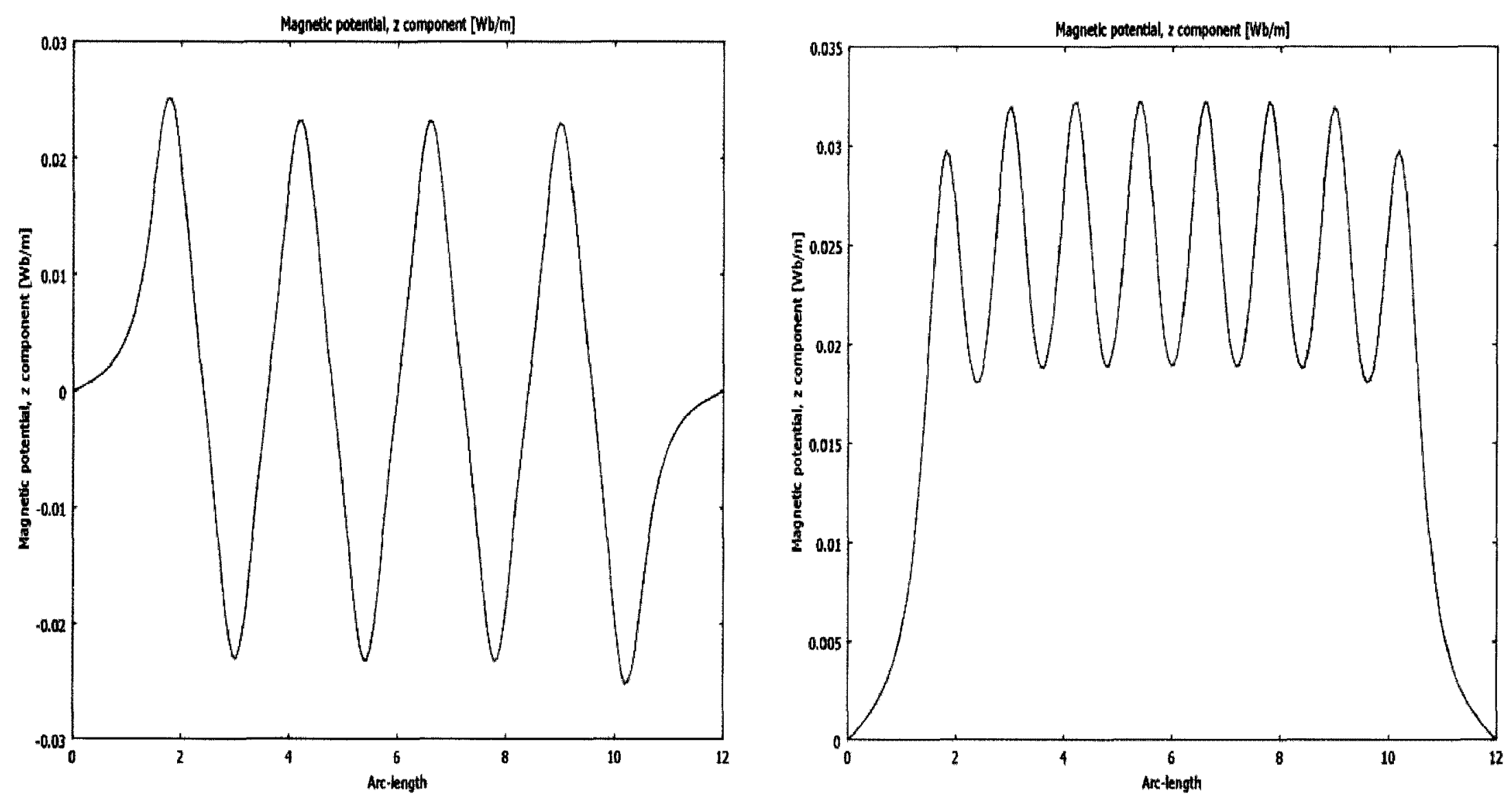

Figure 4-5: Magnetic potential along a line above the track of magnets, as a function of position. The left plot shows bipolar swings when using the alternating configuration. The right plot, for the unidirectional magnet configuration, shows an always-positive magnetic potential and less intense fluctuation. 
a straight line and at a fixed distance from the plane of the wheel.

For maximum stability (i.e. noise immunity), our goal was to have the sensors experience large variations in the magnetic field amplitude as the magnets moved past the sensors. To estimate the wave seen by a sensor as the wheel rotated, we plotted the field strength along the line of travel. Focusing on the part of these curves where fringe effects of the simulation are minimal (i.e. the mid-distance regions; $\mathrm{x}$-axis values of about 4 to 8 $\mathrm{cm}$ ) in both plots of Figure 4-5, we first note that the alternating orientation pattern (left plot) has its field intensity passing through zero whereas the unidirectional pattern (right plot) has a significant magnetic bias potential along the entire path. Furthermore, the alternating orientation configuration (given the same size magnets and the same path distance) yields field magnitude changes about twice that of the unidirectional configuration.

In practice, the strength of the magnets, the sensitivity of the magnetic sensors and the proximity of the magnet travel path need to be adjusted in order to set the point in the cycle at which the sensor turns on and off. Recall, also, that the reed switch turns on when the absolute magnetic field strength exceeds the threshold, so in both configurations we would get one pulse generated per magnet passage. Choosing an appropriate field pattern and threshold will result in a stable design.

The unidirectional configuration (Figure 4-5, right plot) would optimally require placement of the magnetic sensors such that their thresholds are around the bias value (the midpoint between the top and bottom peaks, or about $0.025 \mathrm{~Wb} / \mathrm{m}$ in this model). By making this bias value equal to the threshold (through choice of components and placements), the sensor would switch on and off (as the wheel turns) with a duty cycle of 
approximately $50 \%$. However, relatively small changes off of this bias point (for instance, a $0.01 \mathrm{~Wb} / \mathrm{m}$ change in this model) and the switch could remain always, or almost always, on or off (i.e. duty cycle at, or almost at, $100 \%$ or $0 \%$, respectively), resulting in unstable behaviour .

However, using the alternating direction configuration (Figure 4-5, left plot), a wider range of sensor placements could reliably produce a pulse. Thresholds in the range of $+/$ 0.005 to $+/-0.02 \mathrm{~Wb} / \mathrm{m}$ could do this (recall, again, that the sensor responds to both positive and negative fields). Even with a very sensitive sensor (or stronger magnets, or a closer mounting distance), though the switch would be on for more of the cycle (as the field magnitude exceeds the switch's threshold), it would never result in a $100 \%$ duty cycle. A $50 \%$ duty cycle would be achieved when the arc length where the magnitude of the field is above the designed threshold, is the same as the arc length corresponding to the part of the cycle where the field is below the threshold. In this model, this would be with a threshold of around $0.015 \mathrm{~Wb} / \mathrm{m}$.

To summarize, the simulation results provide us a spatial representation of the magnetic field strength and direction, the latter being a function of the characteristics of the magnets used, their spacing and their relative pole orientations. The alternating pole configuration shows both larger and bipolar fluctuations in field strength, and so was chosen for the proposed design. This configuration offers more stable behaviour in a system where there are slight threshold changes (or small changes in sensor-to-wheel distance) during normal use of the system.

To confirm these findings, magnets where mounted onto a prototype system, in both configurations, and the effects measured with a reed switch placed at various distances 
from the rotating wheel (the prototype system used is described in more detail in Section 4.3.1). Indeed, the alternating polarity configuration showed a significantly larger range of usable distances.

\subsubsection{Sensor Distance from Wheel}

As each magnet on the rotating wheel approaches a sensor, passes below it, and then moves away from it, the magnitude of the field at the sensor increases, peaks and then diminishes (Figure 4-6a). Depending on the strength of the field through the cycle, the switch may be off at all times (cut off), on for part of the cycle (operating region) or on at all times (saturated).

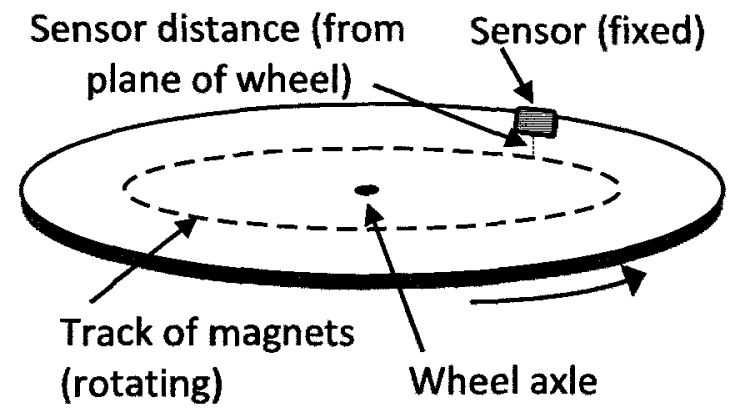

(a)

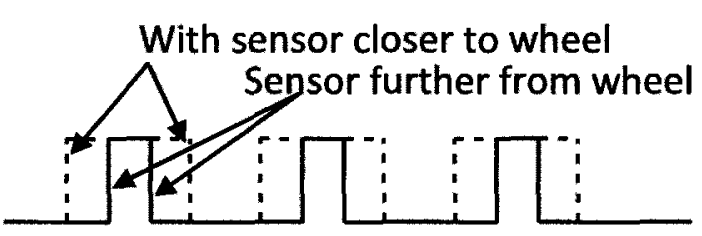

(b)

Figure 4-6: Response of a magnetic sensor mounted above a rotating track of magnets (a). The width of the generated pulses (b) is a function of the distance the sensor is mounted from the plane of the wheel.

Though obtaining a pulse of any width would in theory be sufficient when performing distance measurement by simple pulse tallying, setting the sensor position so as to minimize the chance of losing pulses when subjected to wheel wobble or vibration is necessary to insure robustness in the design.

Wheel wobble and vibration will result in jitter in the sensor output, with pulse widening and narrowing as the wheel gets closer or further from the sensor (as shown in 
Figure 4-6b). Also, slight variations in magnet strengths and track offset positions from one magnet to the next could also, for similar reasons, cause jitter in the output signal.

\subsubsection{Sensor Spacing and Phase Quality}

With the proposed two sensor system, a pulse train is generated from each sensor, but with a phase difference (see Figure 4-7). The phase difference is as a direct result of the spacing of the sensors. As was seen in the analysis above, the width of the pulse will be a result of the distance that a sensor is mounted from the rotating wheel. Too high and the pulses will be narrow or non-existent (sensors always off), too low and the pulses will be very wide or the signal always on.

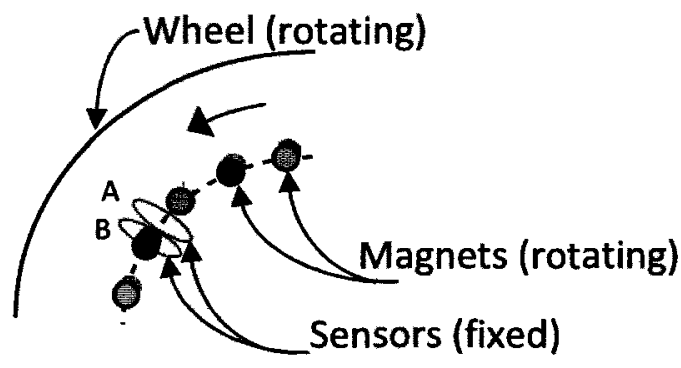

(a)

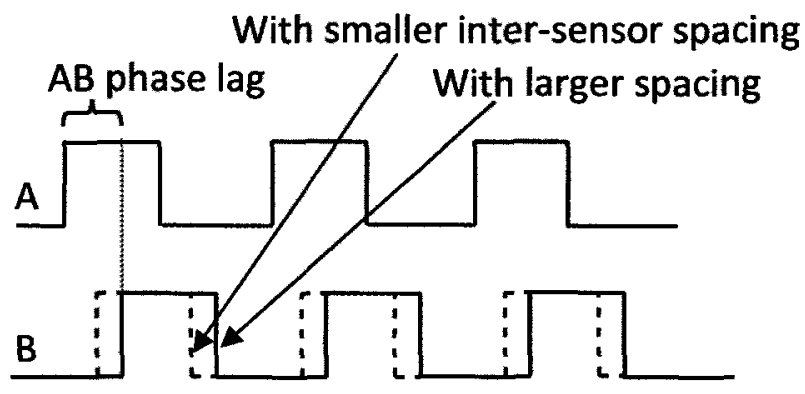

(b)

Figure 4-7: Two reed sensors with a small separation distance mounted above the track of rotating magnets (a) will generate a pair of slightly out of phase pulse trains (b). The phase difference will depend on the separation distance.

The quadrature signal consists of two such pulse trains. These, in addition to having to be robust with respect to their pulse widths, must also have, at all times, a valid phase difference. The phase lag (shown in Figure 4-7b) must be between $0 \%$ and $100 \%$ of the width of the "A" pulse to be valid. Valid phase lag is essential because quadrature encoding uses the polarity of the alternate channel during a signal transition to establish the direction of travel. 
This phase lag depends on the inter-sensor spacing, sensor mounting distance (from the wheel), as well as other factors (including magnet strength and sensor sensitivity), and in an ideal system would be a constant. In practice, due to wheel vibration or wobble, inexact placement of magnets, as well as other factors, there will be some "phase jitter". The nominal phase lag must therefore be chosen so as to be sure that the actual lag will always be within the $0 \%$ and $100 \%$ range. This will insure that a robust quadrature signal is produced.

The optimal phase lag, to obtain best noise immunity, will depend on the duty cycle of the sensor pulses (see Table 4-2). A $20 \%$ duty cycle pulse train from each sensor, for example, should optimally be offset by $10 \%$ of the cycle time (i.e. by $50 \%$ of the pulse asserted time). However, so long as the offset is between $0 \%$ and $20 \%$, a valid quadrature signal will result.

Table 4-2: Range of phase lags for valid quadrature signal.

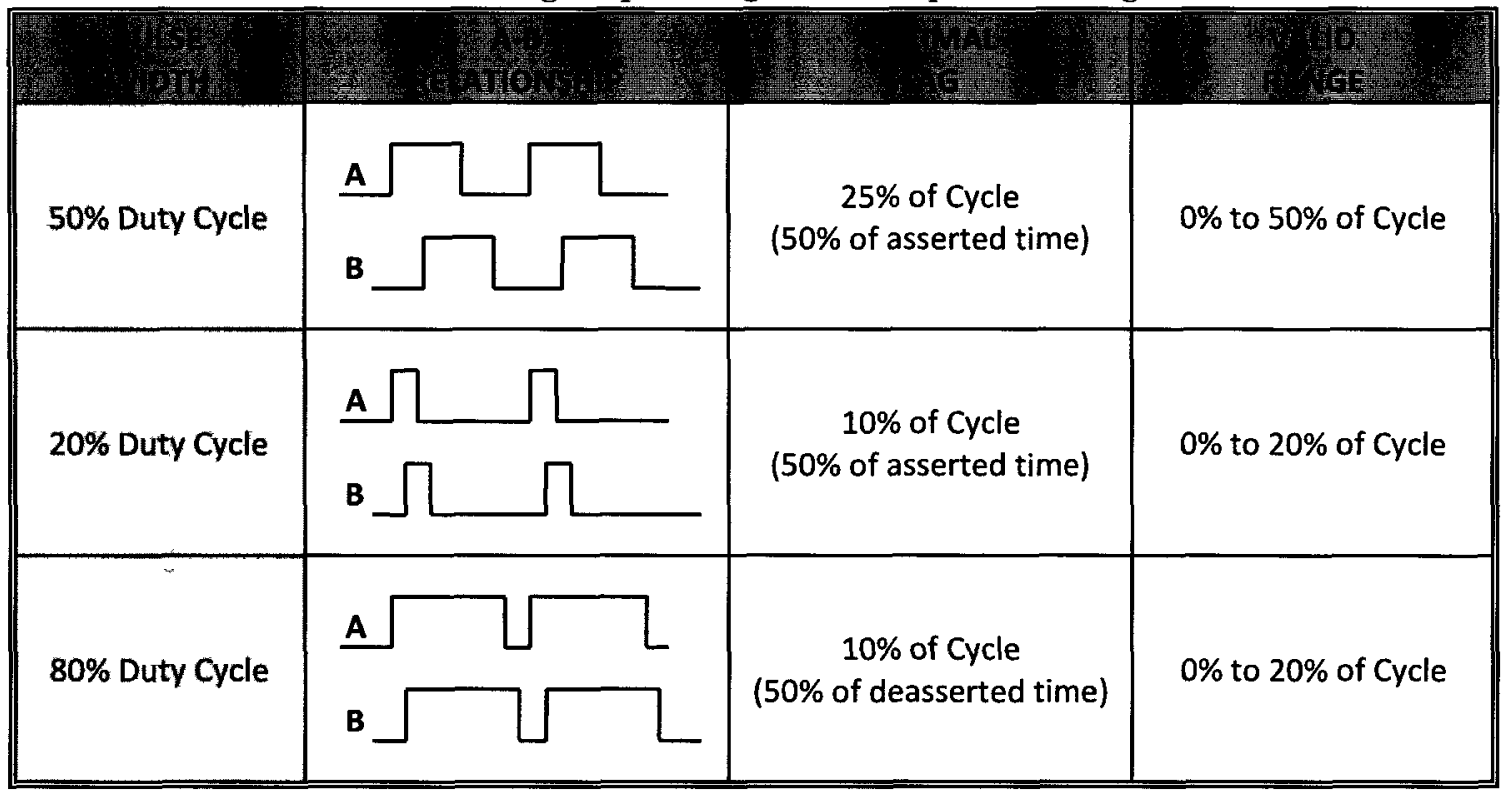


To help in identifying how magnet spacing, mounting distance, and magnet size affect phase lag, a prototype system was used to characterize the relationship. From this analysis, stable configurations can be identified.

It should be noted that when the wheel is turning in the reverse direction, there is a phase lead rather than a phase lag. Nonetheless, the above statements still apply if we substitute the term "lag" with "lead", and so tests to characterize the system need only be done in one direction. In other words, due to positional symmetry, the same response is obtained when going in either direction.

\subsubsection{Measurement Resolution}

Distance measurement resolution will be affected by the number of magnets and the wheel diameter. Where there are $N$ magnets mounted on the wheel, $N$ pulses will be generated for each rotation of the wheel. With equally spaced magnets the resolution is therefore:

$$
R=\frac{2 \times \pi \times r_{w}}{N}
$$

where $\quad r_{w} \quad$ is the radius of the wheel, and

$N \quad$ is the number of equally spaced magnets along a concentric track on the wheel.

Thus, the resolution can be increased by having a smaller diameter wheel or by increasing the number of magnets, though at a higher cost. However, for the application being considered here, we consider the wheel diameter to be a constant (i.e. a design constraint), as the rollator is a production device being retrofitted to include the required sensing and only minimal changes to the underlying rollator design is desired. 
The radius of the magnet track affects the distance between magnets and therefore also affects the maximum achievable resolution. Placing the magnets closer to the edge of the wheel makes for a larger track circumference and therefore place for more magnets. More magnets within a rotation result in better distance resolution.

The maximum number of magnets (and so, the maximum resolution), in practice, is limited by how close the two magnetic sensors can be placed to each other. The magnets will need to be spaced at a distance equal to at least twice the width of the sensor. The maximum achievable resolution for a multiple sensor configuration is therefore:

$$
R_{\max } \cong 2 \times w_{s} \times \frac{r_{w}}{r_{t}}
$$

where $w_{s} \quad$ is the width of the sensor,

$r_{w} \quad$ is the wheel radius, and

$r_{t} \quad$ is the magnet track radius.

To summarize, increasing the number of magnets will increase resolution, limited only by the physical dimensions of the magnetic sensors and magnets. The magnetic sensors, which need to be spaced (center to center) approximately half of the intermagnet distance, will be a limiting factor in maximizing resolution. Increasing the number of magnets will also impact cost.

Depending on the intended purpose of the system, however, a high resolution may not be necessary. In our application, for example, a resolution of half a wheel rotation may be sufficient, in which case only two magnets are needed, lowering costs and reducing the software overhead of count processing. In this case, the resolution would be (referring to value for wheel radius in Table 4-4): 


$$
R=\frac{2 \times \pi \times r_{w}}{N}=\frac{2 \times \pi \times(9.5 \mathrm{~cm})}{2}=30 \mathrm{~cm}
$$

\subsubsection{Distance and Speed Calculation}

In a single sensor design, a wheel with equally spaced magnets (as in Figure 4-2)

generates a pulse as each of the magnets passes beneath the sensor, with each pulse corresponding to a travel distance of

$$
d_{p u l s e}=\frac{2 \times \pi \times r_{w}}{N}
$$

where $d_{p u l s e}$ is the travel distance represented by each sensor pulse,

$r_{w} \quad$ is the radius of the wheel, and

$N \quad$ is the number of equally spaced magnets on the wheel.

After travelling from a position $d_{0}$ to $d_{1}$, to within the resolution of measurement $(R)$, the total travelled distance would be:

$$
\begin{aligned}
\Delta d & =\left(c_{1} \times d_{\text {pulse }}\right)-\left(c_{0} \times d_{\text {pulse }}\right) \\
& =d_{\text {pulse }} \times\left(c_{1}-c_{0}\right)
\end{aligned}
$$

where $c_{1} \quad$ is the cumulative pulse count when at location $d_{1}$, and

$c_{0} \quad$ is the cumulative pulse count at the reference point, $d_{0}$.

The average speed of travel for this time interval is given by:

$$
\begin{aligned}
s & =\frac{\Delta d}{\Delta t} \\
& =\frac{\left(d_{1}-d_{0}\right)}{\left(t_{1}-t_{0}\right)}
\end{aligned}
$$

which, from Eq. 5-4,

$$
=d_{p u l s e} \times \frac{\left(c_{1}-c_{0}\right)}{\left(t_{1}-t_{0}\right)}
$$

where $t_{1} \quad$ is the time at which the object is located at $d_{1}$, and

$t_{0} \quad$ is the time at which the object is located at $d_{0}$. 
Whereas $d_{\text {pulse }}$ is a constant for the system, $c_{0}, c_{1}, t_{0}$ and $t_{1}$ are measured by the system's microcontroller.

For the case where the distances are to be calculated by a quadrature signal, the above analysis stıll applies, but with the cumulative count incrementing or decrementing as per a quadrature decoding rule. Logic for a dual-edge decoding rule is shown in Table 4-3. This counting method, based on pulse transitions rather than pulse counts, will generate a total of four counts per cycle (rather than one count per cycle with one sensor and single edge pulse tallying). Note that, unless the A and B signals both have a duty cycle of exactly $50 \%$ and a phase difference of exactly 90 degrees, the four edge types do not each represent the same travel distance. Nevertheless, the distance represented by the four edges combined corresponds to a full cycle, and so simply dividing the accumulated count by four (during digital processing) will give a correct distance, with the added advantage that we can use equations 5-1 to 5-3, above, as is.

Table 4-3: For quadrature decoding of $A$ and $B$ signals, a unit increment or decrement results from each sensed signal transition. The level of the alternate channel is used to establish if the edge represents a clockwise $(+1)$ or counter clockwise $(-1)$ movement.

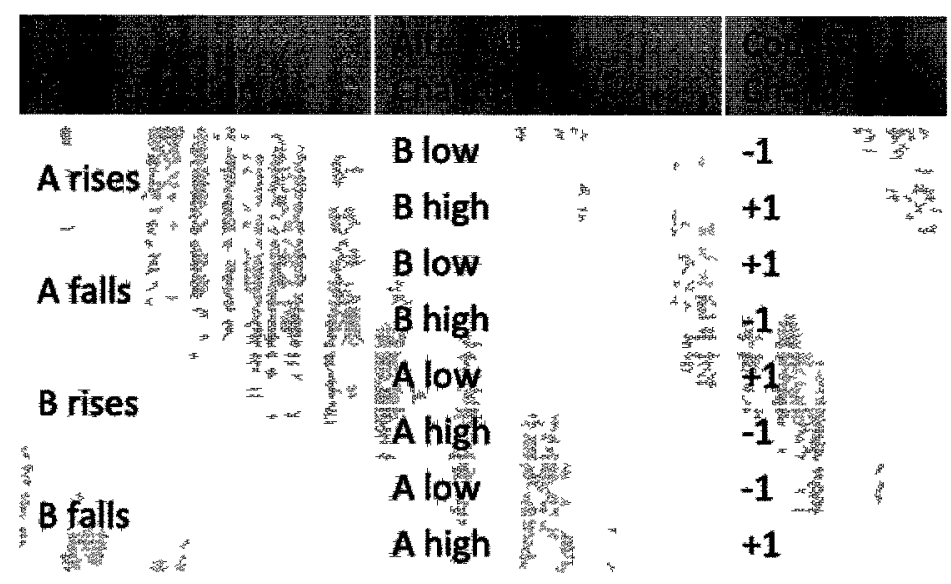




\subsubsection{Movement Artefact Error}

Slight back and forth movements of the user when remaining essentially stationary will cause sensor output (pulses) if the system happens to be near a sensing threshold point. If there are $N$ equidistant magnets mounted on the wheel, there are $2 N$ such threshold points (i.e. $N$ rising field magnitudes and $N$ falling field magnitudes).

With a single sensor and measuring only switch closures, passing the rising field potential threshold corresponds to a travel distance of $R$, the measurement resolution (as given by equation 5-1, above). The expected false distance tallied (or movement artefact error) is:

$$
\begin{aligned}
\mathrm{E}[\text { error }] & =(\text { chance of pulse occuring }) \times(\text { weight of pulse }) \times(\text { frequency }) \times 2 \\
& =\left(\frac{A_{m}}{R}\right) \times R \times F_{m} \times 2 \\
& =2 \times F_{m} \times A_{m}
\end{aligned}
$$

where $R \quad$ is the resolution of distance measurement,

$F_{m} \quad$ is the frequency of the back and forth movement, and

$A_{m} \quad$ is the amplitude of the back and forth movement

So, as a simple example, if the user has a back and forth movement of a fixed distance, say $5 \mathrm{~cm}$, then the probability the threshold being crossed is $5 \mathrm{~cm} / R$, where $R$ is the resolution. If this movement is occurring once a second, then:

$$
\begin{aligned}
\mathrm{E}[\text { error }] & =2 \times(1 \mathrm{~Hz}) \times(5 \mathrm{~cm}) \\
& =10 \mathrm{~cm} / \mathrm{sec}
\end{aligned}
$$

It should be noted (from equation 5-6) that the expected error is independent of wheel size or number of magnets! This is because if there are fewer pulses per revolution, then 
there is a lesser chance of randomly hovering over a magnet (per second), but each pulse represents a correspondingly larger distance.

Also, if the amplitude of movement $\left(A_{m}\right)$ is much less than the resolution $(R)$, then hovering about the threshold point creates large distance tallies though there is a smaller probability that this will occur. And when not hovering about a threshold point, no tallying occurs, of course. Thus, expected error ( $E[$ error $]$ ) represents average distance tallying when randomly stopping at different wheel positions and hovering there.

With quadrature (dual sensor) wheel motion sensing, we know the direction of the wheel's rotation on generation of each pulse. So, when hovering, pulses representing "forwards" movement cancel those representing "backwards" movement, resulting in no movement artefact error.

\subsection{Methods}

\subsubsection{Prototype System}

A bench prototype system was built to characterize an implementation of the proposed system, with the goal of assessing the achievable robustness of the design as a function of configuration. Wheel vibration and wobble affects the quality of pulses generated by the reed switches. In a two-sensor system, such as the one being proposed, it also affects the validity of the quadrature signal. By choosing and installing the components of the system such that this noise is bounded within known limits, a robust system is achieved.

A testing fixture (Figure 4-8) was built to measure the effects of changes in sensor mounting distance from the wheel, separation of the sensors, and magnet size. A prototype wheel was created out of layers of board and two clear outer plastic discs, with 
holes punched into the board for placement of the magnets. These were assembled and mounted onto a motorized rotary system (a side mounted drill press). We chose small powerful rare-earth magnets as well as sensitive and easily mountable reed switch sensors for the design (see Appendices A.5 and A.6 for a description of the parts used). Finally, an adjustable bracket was used to hold the sensors.

To characterize the sensor and to make measurements of pulse quality, a single reed switch was mounted "above" the track of rotating magnets (Figure 4-8b). That is, it was mounted at the same radial distance from the axis of rotation as the track of magnets. To generate and characterize a quadrature signal, a second sensor was then mounted at the same distance from the wheel, as well as the same distance from the axis of rotation (Figure 4-8c).

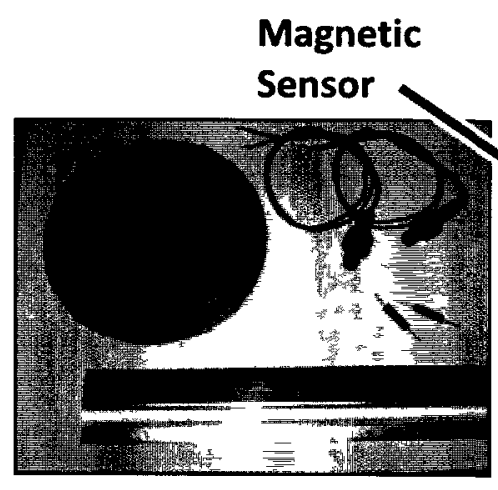

(a)

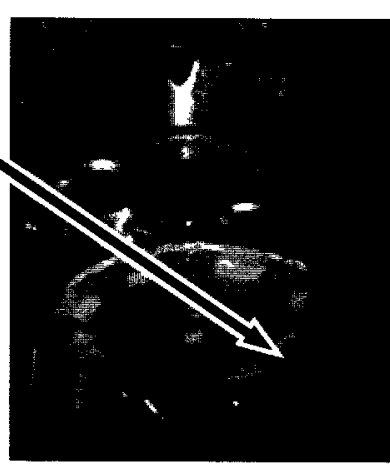

(b)

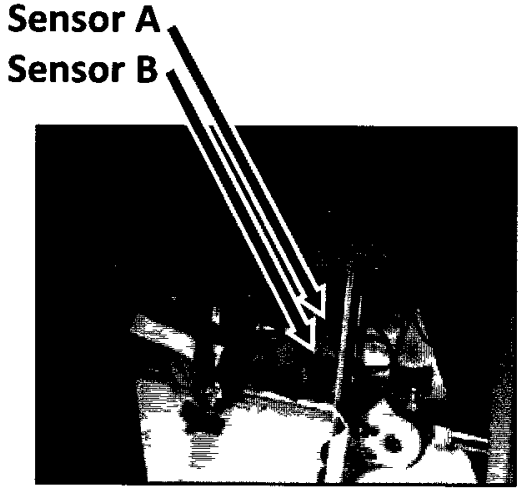

(c)

Figure 4-8: Test system consisting of a rotating disk with countersunk magnets (a) and a magnetic sensor mounted on a fixed bracket (b). To assess the effect of separation distance, a second magnetic sensor was mounted next to the first one (c).

Table 4-4 gives the specifications of the prototype and the rollator systems. Though the dimensions of the prototype were different than those of the rollator being retrofitted, the characterization and analysis is expected to be similar and therefore useful for initial design purposes. 
Table 4-4: Specifications of the prototype and rollator systems.

\begin{tabular}{|l|l|l|}
\hline Diameter of wheel & $12 \mathrm{~cm}(4.75$ inches) & $19 \mathrm{~cm}$ (7.5 inches) \\
\hline Diameter of magnet track & $10 \mathrm{~cm}(4$ inches) & $12.5 \mathrm{~cm}$ (5 inches) \\
\hline Numbér of magnets & 10 & 10 \\
\hline Inter-magnet distance - degrees & 36 degrees & 36 degrees \\
\hline Inter-magnet distance - arc length & $3.1 \mathrm{~cm}(1.2$ inches) & $3.9 \mathrm{~cm}$ (1.5 inches) \\
\hline Diameter of small magnets & $0.64 \mathrm{~cm}(0.25$ inches) & - \\
\hline Thickness of small magnets & $0.64 \mathrm{~cm}(0.25$ inches) & - \\
\hline Diameter of large magnets & $1.27 \mathrm{~cm}(0.5$ inches) & $1.27 \mathrm{~cm}$ (0.5 inches) \\
\hline Thickness of large magnets & $0.32 \mathrm{~cm}(0.125$ inches) & $0.32 \mathrm{~cm}$ (0.125 inches) \\
\hline
\end{tabular}

A simple circuit was assembled to measure the state of the reed switches. This type of magnetic sensor has normally-open contacts which close when in the presence of a magnetic field. To monitor its state, one side of the switch was grounded and the other side connected to a pull-up resistor (see Figure 4-9).

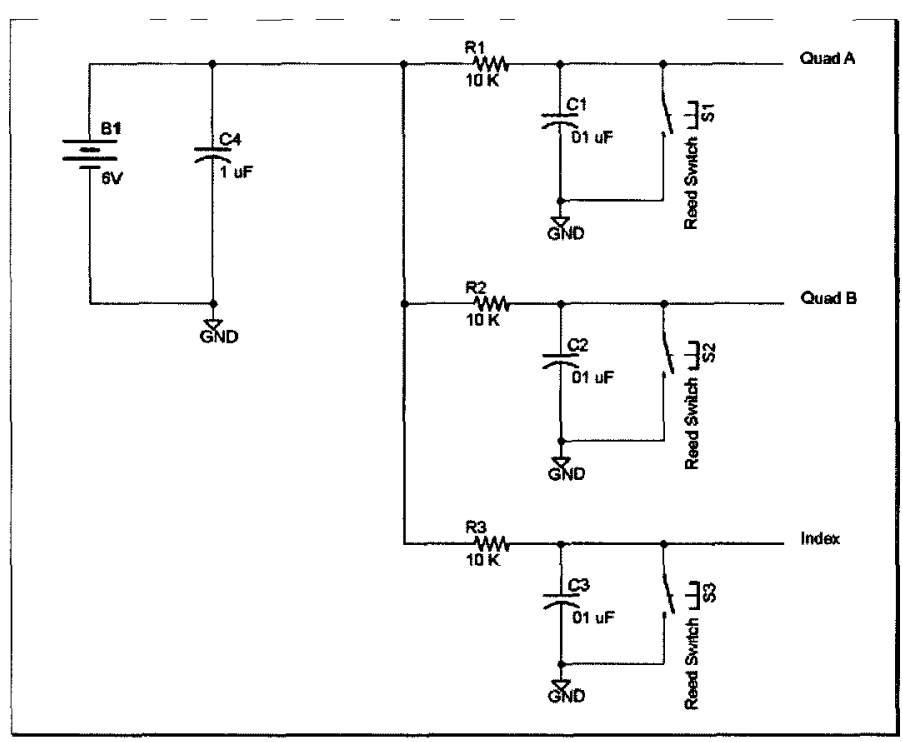

Figure 4-9: Three reed switches are used in testing the system. S1 and S2 are above the track of magnets, generating the quadrature $A$ and B signals. S3 generates one pulse per rotation, to help isolate and characterize individual magnets on the wheel. 
Capacitors were added to remove contact bounce and to reduce noise in the supply rail. Since in this configuration falling edges were sharpest (whereas rising edges had an $\mathrm{RC}$ time constant decay), triggering and timing measurements were made from falling edges wherever possible. Where rising edges needed to be measured (e.g. for pulse width), the time at the start of the rise was used.

\subsubsection{Pulse Quality Measurement}

A first test was performed to determine the effect of varying the distance between the sensor and the plane of the wheel. With the sensor fixed at various (arbitrarily chosen) distances, the wheel was spun and the width of the sensor pulse measured with a digital storage oscilloscope (Tektronix TDS 1002). The pulse-to-pulse period was also measured and the ratio of the former to the latter used to calculate the duty cycle. Duty cycle gives us a measure of pulse quality and is independent of the speed of wheel rotation (for speeds of interest).

However, as we began to make these measurements, we observed that the duty cycle varied considerably from one magnet to the next (as the wheel rotated). This was attributed to wheel wobble, inexact placement of the magnets (by either not all being exactly the same distance from the wheel's axis, or not being perfectly spaced along the track's circumference), and perhaps slight differences in strength between the magnets. To address this, an additional magnet and magnetic sensor were added near the drill head (away from the wheel), to generate one pulse per rotation. This "index" signal was used to trigger the oscilloscope display and isolate measurements to a single magnet. All measurements made were for this single magnet. 


\subsubsection{Measurement of Quadrature Signal Validity}

A second test consisted of observing, with two sensors mounted, the relationship between the respective signals (A and B) and evaluating whether they conformed to requirements for being a valid quadrature signal. The two sensors were mounted directly "above" the track of magnets, first taped together (i.e. right next to each other) and then at progressively greater separations up to a center-to-center distance close to the intermagnet spacing. We measured the A-to-B phase lag (as shown in Figure 4-7b) as a function of sensor separation. The process was then repeated, iterating with both a different size magnet and with changes in sensor distance from the wheel.

For each of the iterations, separation distance of the sensors was first measured, then the wheel was put into rotation and the A-to-B lag time was measured. The asserted time and period of A were also recorded. The lag time as a percentage of cycle period and the pulse-asserted time as a percentage of period (i.e. the duty cycle) were then calculated and the ratio plotted as a function of sensor separation. Plotting the lag to pulse width ratio (as opposed to the absolute value of the lag) expresses the A-to-B relationship in a more meaningful way (i.e. $0 \%$ to $100 \%$ is valid, other points are not - see Table 4-2), these values being independent of wheel rotation speed.

\subsubsection{Measurement of Wheel Wobble and Vibration}

The goal of the speed measurement system design process presented here is to identify components and configurations in which the measurement signals will be consistently valid, under real world operating conditions. By knowing the maximum wobble or vibration to which the magnet-sensor pair will be subjected, we can choose a 
configuration which is stable throughout the full range of magnet-to-sensor separation distances.

Wheel play, typically the result of loose bearings (or a loose interface between the axle and the wheel), is measured with the wheel stationary. The wheel is "pushed" and then "pulled" lightly in the axial direction, at the edge of the wheel, and the total displacement of the magnets is recorded.

Wheel wobble, on the other hand, manifests itself when the wheel is rotated. It results from the wheel axle not being perfectly perpendicular to the wheel's surface. What is observed is that, as the wheel rotates, the wheel surface moves back and forth in the direction of the axle's axis. The amplitude of the back-and-forth movement during one rotation is the wobble.

Finally, magnet misalignment represents the inexact axial placement of magnets on the wheel. In the case of the prototype system, the magnets were "sandwiched" between two clear plastic disks, so the magnet surfaces were all in line and so there was no misalignment. The rollator, on the other hand, had the magnets glued onto the rim of one of its wheels. Some of magnets had a bit more glue holding them in place than others, the result being a misalignment (from the "highest" to the "lowest" magnet).

All of these measurements were taken at the magnet track radius.

Summed up, these three values represent the maximum misalignment in magnet-tosensor distance which must be accommodated by the system while still generating a consistent and valid quadrature signal. 


\subsection{Results}

\subsubsection{Pulse Quality}

To characterize the quality of pulses generated during wheel rotation, duty cycle as a function of sensor distance from the wheel, and magnet size, was plotted (Figure 4-10). Three measurements were made (and plotted) for each setting. As the curves suggested by the data points intersected the axes perpendicularly, the points were fitted with an ellipse function using a least-squares error algorithm. The fit was good and so these curves were used for interpolation during the design process (Section 4.5).

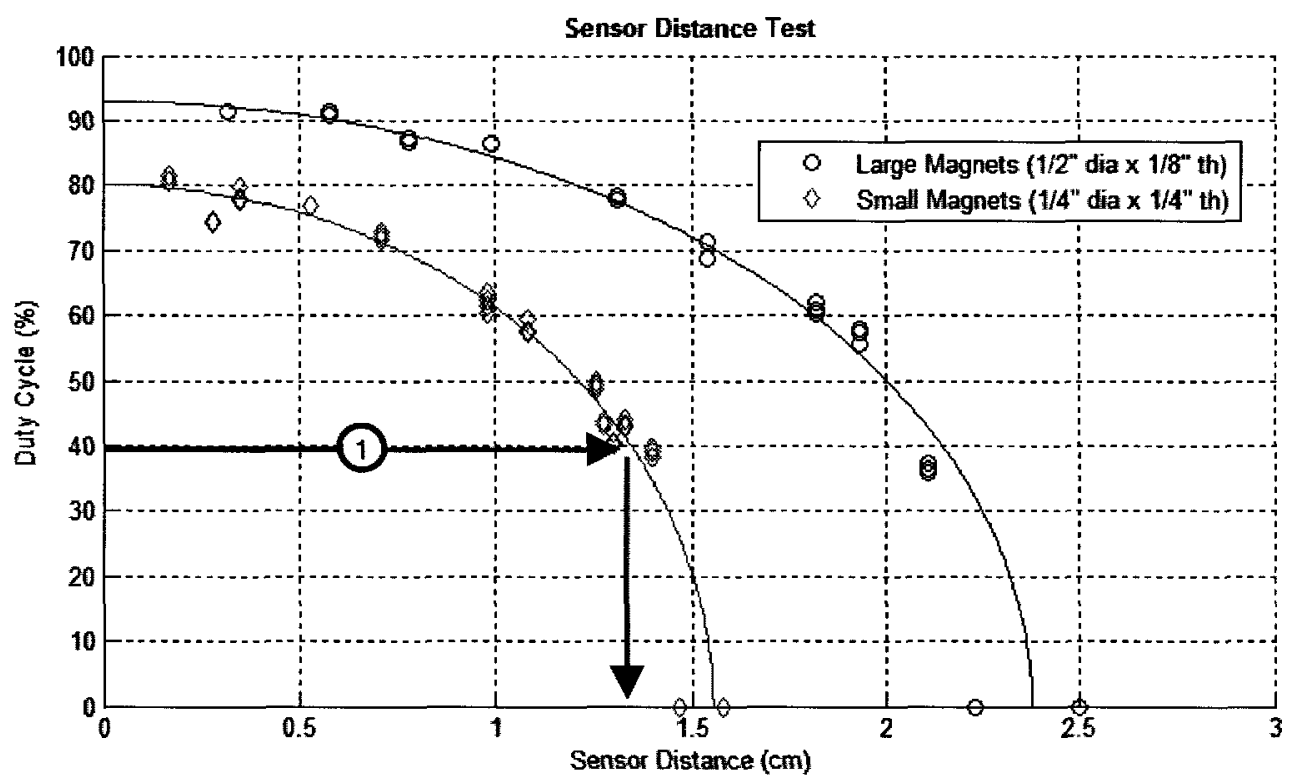

Figure 4-10: On versus off time (duty cycle) of magnetic sensor for rotating wheel with ten magnets. Plotted as a function of distance mounted from wheel and size of magnets used.

The larger magnets (points represented by blue disks in the plot) exceeded the sensor's threshold for a longer part of the cycle than the smaller magnets. This was attributed to their having a greater magnetic strength (due to their higher volume) as well as being wider (i.e. larger diameter). At $1 \mathrm{~cm}$, for example, the threshold was exceeded 
for about $85 \%$ of the cycle time for the large magnets versus $60 \%$ of the time for the small magnets. In both cases, as expected, as the sensor was moved further from the wheel, the sensor switch closed for a briefer and briefer time until a critical point was reached where the threshold was never exceeded (occurring here at around $1.5 \mathrm{~cm}$ for the small magnets and $2.2 \mathrm{~cm}$ for the large magnets).

To summarize, we observed that using a larger magnet shifts the sensor response curve to the right. Conversely, the sensor needs to be mounted closer to the wheel for a similar response if using a smaller magnet.

\subsubsection{Quadrature Signal Validity}

For the second test, measurements were made with the sensors mounted at distances from the wheel which resulted in duty cycles of about $50 \%$ and $75 \%$ (as established from the test above). Measurements were also to be made for signal duty cycles of $25 \%$, but this was reconsidered due to signal instability at these settings (steep slope region of curves in Figure 4-10). As in the previous test, three measurements were made (and plotted) for each setting (Figure 4-11).

The phase quality in these plots is expressed as "Ratio of A-to-B Lag Time to A Asserted Time". This is the lag time of the B signal with respect to the A signal, divided by the lesser of the A high time or A low time, and expressed as a percentage. For example, a measured lag time of $6 \mathrm{~ms}$ when the A pulse width is $10 \mathrm{~ms}$ (and the period of A $>20 \mathrm{~ms}$ ) would be plotted as a phase lag ratio of $60 \%$. By choosing this measure, valid quadrature signals occurs when the ratio is between $0 \%$ and $100 \%$ (Figure $4-3 \mathrm{c}$, as an example, illustrates a phase lag ratio of $50 \%$ ). It is because quadrature decoding uses the 

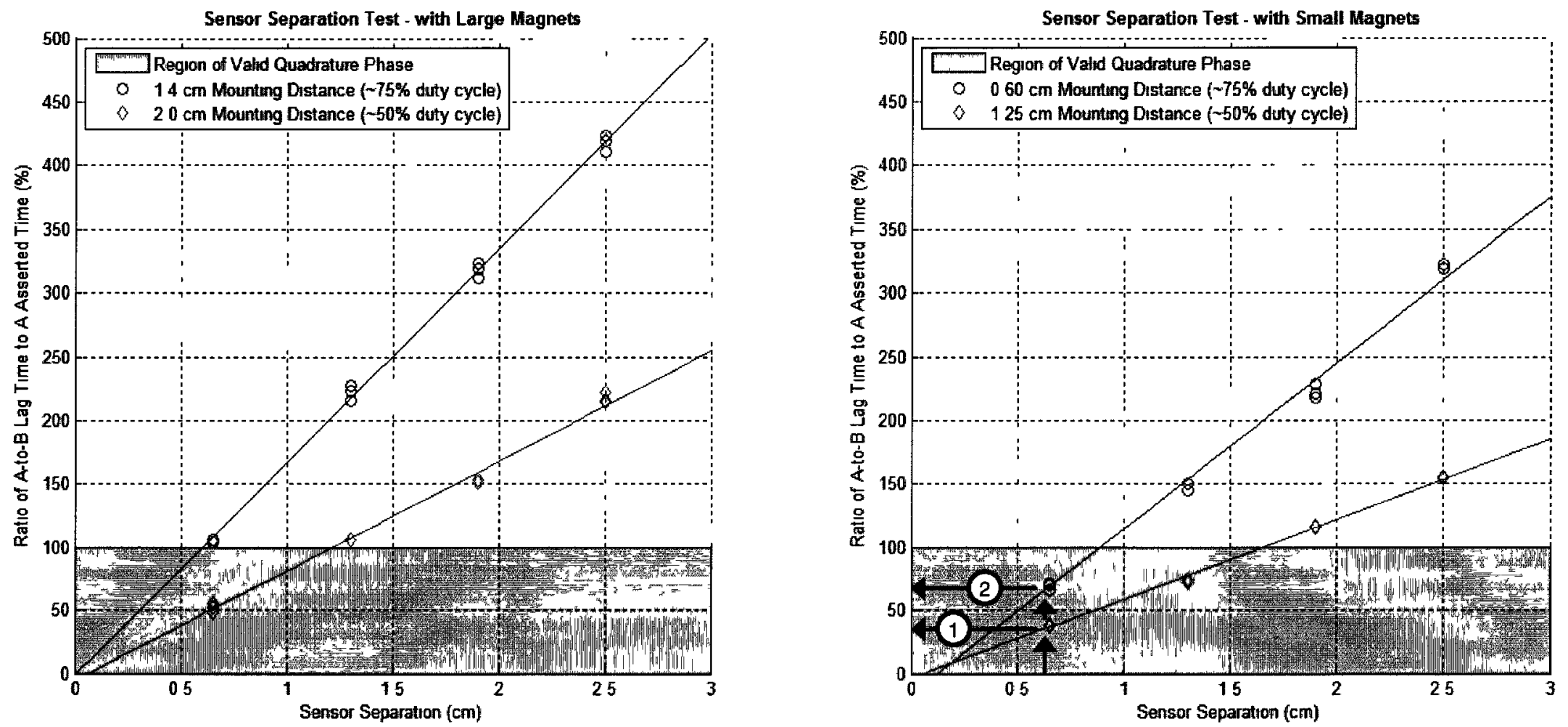

Figure 4-11: Effect of separation distance between two sensors on the quality of the quadrature signal. Phase quality is plotted as the ratio of A-to-B phase lag time to " $A$ " pulse-asserted time, as a function of sensor separation, sensor mounting distance from the wheel, and magnet size. Note that the signal is only usable when the phase lag ratio is between $0 \%$ and $100 \%$. 
level of the B signal during transitions of the A signal (and vice versa) to establish movement and direction that there must be an overlap to obtain valid quadrature coding.

The data points fit well to a set of first order line passing (roughly) through the origin. This was as expected, as the separation of the sensors should, intuitively, be directly proportional to the phase difference of the pulses coming from the sensors. These straight lines were used in interpolating values between the data points, to assist in the design of the speed-distance monitoring system (Section 4.5).

Best quadrature signals were obtained when the duty cycle was around $50 \%$ (red diamonds), and with small sensor separations. The lowest possible sensor separation was limited by the physical width of the sensors, which in this case was $0.65 \mathrm{~cm}$.

\subsubsection{Magnet Positioning Error}

Errors which cause undesired variances in the magnet-to-sensor distance as the wheel rotates were measured (see Table 4-5). The main errors identified for this study were wheel play, wheel wobble and magnet misalignment.

Table 4-5: Measured wheel play, wheel wobble and magnet misalignment.

\begin{tabular}{|l|l|l|}
\hline & & \\
\hline Wheel Play & $1.0 \mathrm{~mm}$ & $1.4 \mathrm{~mm}$ \\
\hline Wheel Wobble & $1.9 \mathrm{~mm}$ & $2.0 \mathrm{~mm}$ \\
\hline Magnet Misalignment & $0.0 \mathrm{~mm}$ & $0.6 \mathrm{~mm}$ \\
\hline Maximum positional misalignment & $2.9 \mathrm{~mm}$ & $4.0 \mathrm{~mm}$ \\
\hline
\end{tabular}




\subsection{Discussion}

Measurement systems using magnets and reed switches to sense proximity, such as what is being studied in this chapter, are frequently assembled in a casual manner, trying out a few components and "eyeballing" the settings until what appears to be a stable signal is obtained. Though this may be satisfactory for some non-critical applications, an engineering approach to design avoids some potential problems:

1. A marginally stable system, in which pulses are generated most of the time but occasionally are missing, will result in incorrect distance and velocity measurements. The distance errors will accumulate and could result in significant absolute errors over time.

2. Building a two-sensor quadrature encoded system can be particularly risky because an improper phase relationship between the two signals will result in the count being incremented in the wrong direction! And this phase error could simply be the result of one or both signals having too narrow a pulse.

3. Because a marginally stable system appears to operate most of the time, it is sometimes difficult to notice that there is even a problem.

Unfortunately, as previously mentioned, the required engineering specifications for both magnets and reed switches are lacking. Furthermore, the complexity of the physics of a sensing system similar to what is being presented here is such that it is difficult or impossible to accurately calculate the sensitivity and response (Hamlin Inc., 2007) (LaBarge \& Gutierrez-Miravete, 2008). Characterizing the system, as is being proposed in this chapter, is a practical way of dealing with this engineering challenge. 


\subsubsection{Design of a Simple Pulse Tallying System}

In a single sensor design, we need simply choose a nominal wheel-to-sensor-track mounting distance such that the known maximum misalignment in the wheel-to-sensor distance will never result in missing pulses. Missing pulses can be due to either the sensor being too close to the magnets' trajectory and remaining saturated (always on) for more than one inter-magnet period, or due to the sensor not being close enough to the magnets' trajectory and the magnetic field intensity never reaching the sensor's threshold.

The total wobble (as defined in section 5.4.3) together with a chosen nominal wheelto-sensor-track mounting distance provides us with a known bounded range of wheel-tosensor-track distances. For instance, if for the bench prototype, we choose to use small magnets, and choose a nominal sensor mounting distance of $0.8 \mathrm{~cm}$, then we know (from Table 4-5) that the wheel-to-sensor-track distance has a range of 0.5 to $1.1 \mathrm{~cm}$. Figure 4-10 shows us that the resulting pulses will vary from about a $55 \%$ to $75 \%$ duty cycle and so, indeed, will remain stable in the worst case. Being well within $0 \%$ to $100 \%$ also means that this system's configuration has a wide tolerance for drift and other errors.

\subsubsection{Design of a Quadrature Encoded System}

A two-sensor quadrature design requires that, in addition to reliably obtaining pulses from each of the two sensors, the pulses maintain an appropriate overlap. The effect of wobble is that the sensor-to-magnet-track distance varies, resulting in changes in the A and B pulse widths. An A-to-B phase lag ratio of between $0 \%$ and $100 \%$ must be maintained in order for the quadrature signal to remain valid. 
Once a nominal sensor-to-magnet-track mounting distance has been chosen and we know the range of sensor-to-magnet-track distances (as discussed in the previous section), we can then refer to Figure 4-11 to see what the range of phase responses are for that configuration. If the range is within $0 \%$ and $100 \%$ phase lag region, then the configuration is stable.

Continuing with the previous example, with a nominal sensor-to-magnet-track distance of $0.8 \mathrm{~cm}$, a total wobble of $2.9 \mathrm{~mm}$, using small magnets and adjacently spaced sensors, there is a duty cycle range in the generated pulse of between $55 \%$ and $75 \%$. We can infer that for this range of duty cycles, we can expect to have a range of about $40 \%$ to $70 \%$ phase lag ratio (labelled (1) and (2) in Figure 4-11, respectively). Since this range is well within the $0 \%$ to $100 \%$ stability range, it is a good design. Had we, on the other hand, chosen a slightly larger nominal sensor-to-magnet-track distance (say, $1.0 \mathrm{~cm}$ ), there would have been a range of duty cycle values of about $40 \%$ to $70 \%$ ). However, at the $40 \%$ point $(1.3 \mathrm{~cm})$ we are at the steep portion of the curve (indicated by (1) in Figure 4-10) and perilously close to losing our pulse!

Table 4-6 summarizes the suggested configuration for the prototype system. The test system was set up with the configuration just discussed, and the resulting signal for the system observed (Figure 4-12). Nearly a complete rotation of the wheel is shown (8 out of the 10 pulses are displayed). The " $A$ " channel is the top trace and the "B" channel, the bottom trace. We note some variances in the pulse width from one magnet to the next, yet all have a healthy overlap between the A and B channels and no missing pulses. Of course, this is the output of an unstressed system, and so to confirm the robustness of the design the system would need to be analysed while subjected to maximum wobble. The 
prototype system setup was as shown in Figure 4-8c.

Table 4-6: Design parameters and specifications for a robust magnetic quadrature encoder.

\begin{tabular}{||l|l|}
\hline \multicolumn{1}{|l|}{ Magnet Size } & Small (0.25 inch dia x 0.25 inch th) \\
\hline $\begin{array}{l}\text { Nominal Sensor to Magnet } \\
\text { Track Mounting Distance }\end{array}$ & $0.8 \mathrm{~cm}$ \\
\hline Sensor Separation & $0.65 \mathrm{~cm}$ (minimum spacing) \\
\hline Total Wobble & $2.9 \mathrm{~mm}$ \\
\hline Sensor to Magnet Track Distance & 0.5 to $1.1 \mathrm{~cm}$ \\
\hline Pulse Duty Cycle & $\sim 55 \%$ to $75 \%$ \\
\hline Pulse Quality & Valid \\
\hline Ratio of Phase Lag to Asserted Time & $\sim 40 \%$ to $70 \%$ \\
\hline Quadrature Quality & Valid \\
\hline
\end{tabular}

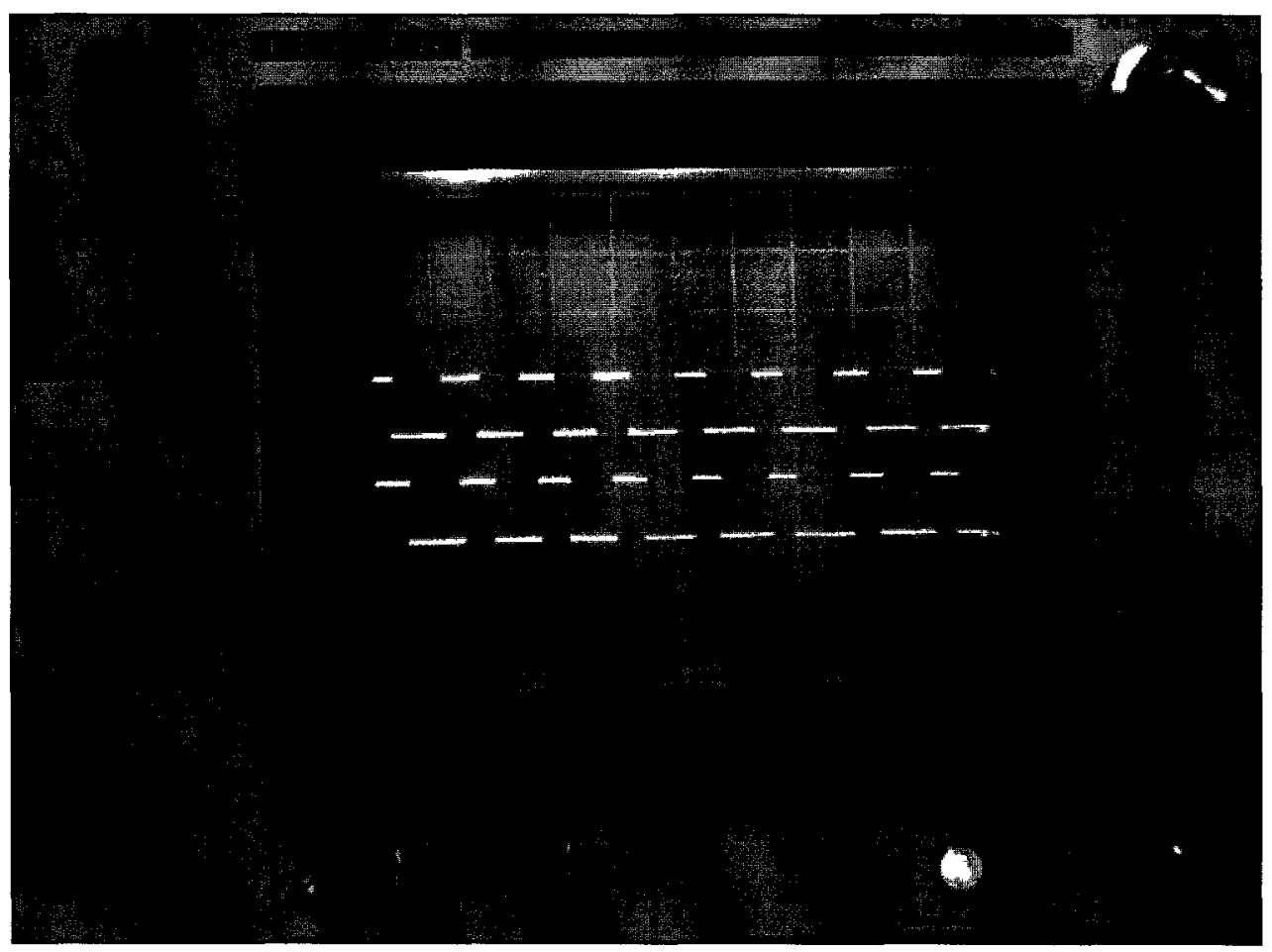

Figure 4-12: The quadrature signal measured with the wheel rotating at a constant speed. The duty cycle and phase difference were set by adjusting the sensor-to-wheel distance and spacing of the sensors, respectively. 
The process outlined in this section, so far, focused on how to use the characterization test results to validate a choice of sensor mounting positions, sensor separation and magnet size, but did not instruct on how to choose these in the first place. Though not the only approach, the process used by the author was an iterative one. This involved choosing a nominal sensor distance (using Figure 4-10) such that, with the known maximum wheel wobble, "healthy" (about 50\% to $75 \%$ duty cycle) pulses resulted. Depending on the desired range of mounting distances (given the constraints of the rollator design), the size of magnets to use can be chosen. The centre-to-centre separation of the "A" and "B" sensors is chosen using Figure 4-11. In all cases, the smallest separation $(0.65 \mathrm{~cm}$, i.e. when they are mounted adjacent to each other) showed the best response, and so this was used. Finally, we verify that with the range of pulse duty cycles, the quadrature signal has a phase ratio of less than $100 \%$. Here, a choice of small magnets may be necessary if the system has large maximum wobble. If the selected configuration in unstable or marginally so, the process is repeated with a different sensor mounting distance. Finally, if no stable configurations can be found, the system's geometry, magnet size or choice of sensors may need to be changed, in which case the system would need to be recharacterized (as per section 4.3).

\subsection{Conclusion}

The design presented in this chapter is similar to the original single sensor pulse counting design, but with one extra magnetic sensor installed at the same radial distance and inline with the first sensor. Both sensors are mounted at the same radial distance as the 
track of rotating magnets on the wheel. The output is a two-bit quadrature signal, a twobit Gray code suitable for up-down counting by a microcontroller.

An empirical design process is described to establish the best mounting distance of the sensors from the plane of the disk, as well as the best spacing between the sensors. This process consists of characterizing the wheel-magnet-sensor system to assess the potential robustness of the system for quadrature encoding.

To obtain a stable quadrature solution, the jitter (due to variances in magnet to magnetic sensor distances) will need to be much less than the offset between the pulses generated by the sensors. So there are three aspects to maximizing performance: (1) reducing magnet-sensor spacing variances during normal use (e.g. wheel wobble) to reduce pulse width jitter, (2) having a large cycle distance, such that the width of each pulse (generated by magnets and magnet separations) is wide (significantly wider than the jitter), and (3) choosing components and their placement such that a nominal duty cycle of around $50 \%$ is achieved.

For our prototype, a feasible configuration was identified and tested. For cases where a feasible configuration for a quadrature encoded system is not possible, the proposed empirical design process may still identify a robust simple pulse tallying (1-bit) solution.

The proposed design improves on the previous single-sensor approach by providing, in addition to the existing measurement of "absolute" distance travelled (that is, the total of forward movement plus backward movement), a "net" distance travelled (the total of forward movement minus backward movement). Having both net and absolute distances gives information that may contribute, possibly in combination with other sensed data from the Smart Rollator, to an improved product for users. 
Being able to independently sense forward and backward movement also allowed us to eliminate error due to false counts. This type of error may occur when, with the rollator device essentially at rest, one of the wheel magnets hovers below the magnetic sensor, generating false counts.

In the future, should angular direction of movement also be needed, an additional rotary encoding sensor could be mounted onto one of the front wheel's swivel, or an electronic compass (magnetometer) installed at a convenient location on the rollator frame. This orientation signal, combined with the forward/backward displacement signal, could be used to calculate trajectory information. 


\section{Chapter 5: Conclusion}

\subsection{Summary of Research}

The objective of the Smart Rollator project is to enable health care monitoring and quantify rollator usage by embedding electronic sensing and recording capabilities onto rollators. The research presented in this thesis focused on the design of two of the sensor subsystems in the Smart Rollator: ECG acquisition for heart rate monitoring, and travel speed/distance measurement.

\subsubsection{ECG Acquisition}

Embedding ECG monitoring into a rollator presented several interesting design challenges. Since use of a rollator is intermittent and since one objective of the project is to make non-obtrusive measurements, using popular wet electrodes was not an option. Large dry electrodes in the handles, however, provided a convenient way to acquire the signal. To evaluate the differences between these electrode types and assess the possible impact on an ECG amplifier design for the rollator, a set of dry grip electrodes using tin sheet metal was prototyped and its performance characterized.

Another design requirement was to acquire the ECG signal using two electrodes (i.e. without a ground or reference drive electrode). An alternate approach for making the body's common-mode (CM) potential (or at least its DC potential) close to that of the ECG amplifier was needed. A CM input shunt circuit, similar in design to what is used in 
subscriber line telephone systems, was employed to present low CM input impedance while maintaining moderately high differential-mode (DM) input impedance.

Finally, as signal acquisition is to be performed during periods of typical activity (e.g. when the user is walking), we expected significant motion artefact from electrode movement as well as significant amounts of EMG noise from muscle activity. The approach taken in the proposed design was to perform a minimal amount of noise filtering in the electronics (just enough to avoid saturation during typical operation) and then perform signal processing digitally, where there are more choices, better performance is achievable and the configuration is easier to change. Digital signal processing of ECG waveforms is a well researched area and not within the scope of this research.

The proposed ECG amplifier circuit was built and characterized. Its input impedance and frequency response was compared to that of a more conventional three-electrode amplifier (built using similar components, running off the same supply voltage and using the same grip-style dry electrodes).

Some pilot user tests were performed on four healthy volunteers, using the proposed two-electrode proposed amplifier and, as a comparison, using a built three-electrode conventional amplifier. The subjects were asked to stand normally while holding onto the rollator handles (which had the electrodes mounted on them), then to walk slowly with the rollator, and finally, to grip as tightly as possible onto the handles. The recorded results were compared between circuits and among individuals. The advantage of the proposed amplifier is that it enables a two-electrode interface, more appropriate for the Smart Rollator application. Finally, as a preliminary analysis, some simple digital 
filtering was performed on one of the ECG recordings to qualitatively assess what ECG quality may be achievable at each of the activity levels, and if and when heart beat extraction is feasible using the designed system.

\subsubsection{Speed/Distance Measurement}

A previous method of speed and distance monitoring was re-examined, to assess robustness of the design and to add forwards/backwards discrimination. A simple system, consisting of a magnetic reed switch attached to the frame near one of the wheels and a number of magnets mounted onto the wheel, created a stream of pulses as the rollator was moved. This method, however, could not detect the direction of wheel rotation and so occasionally, when "hovering" around a threshold point, generated a stream of pulses (which resulted in false travel measurements).

Adding an additional sensor, also above the rotating track of magnets, generated a second stream of pulses similar to that of the first sensor but with a phase difference. If appropriately positioned, the overlap of the pulses could create a quadrature encoded signal. The key to this magnetic rotary encoder design working reliably in practice, however, is in obtaining pulses consistently, and with known widths, given inexact wheel rotation (i.e. due to wobble and play) and inconsistencies of certain system parameters (such as magnet strengths and positions).

An empirical process for evaluating the placement of magnets and sensors to obtain robustness in design and to meet required resolution requirements was outlined. It is being proposed that this process be used to identify which combination of choice of components (magnets and sensors) and placement of sensors will create a robust speed- 
distance measurement system. The process consisted of characterizing a prototype system, with different size magnets, as well as different sensor distances (from the wheel) and separations (from each other). By plotting and analysing the response, robust configurations can be identified. To test this, a prototype system was built, characterized, and from this a stable configuration identified.

\subsection{Main Conclusions}

\subsubsection{ECG Acquisition}

The dry grip electrodes studied had significantly lower skin-to-electrode interface impedance than typical paste-on wet electrodes. This was attributed primarily to their large contact area. The low impedance (about $25 \mathrm{~K} \mathrm{Ohms} \mathrm{at} 10 \mathrm{~Hz}$ ) should result in a performance advantage, including less CM interference caused by electrode impedance imbalances and less signal loss (attenuation) in the skin-to-electrode interface.

Though the electrode impedance was low, this was mostly due to capacitive effects. This implies that such an electrode is more prone to motion artefact, and so this was taken into account during the design of the ECG amplifier. Circuits to block DC and to attenuate low frequencies $(<5 \mathrm{~Hz})$, in order to avoid circuit saturation, were included in the design.

Correlation between intensity (level of) activity and total noise amplitude (motion artefact and EMG noise) was apparent in the results of the pilot user test. The correlation was equally apparent when using the proposed two-electrode ECG amplifier or a more conventional three-electrode amplifier design. As for noise management, though both 
circuits showed similar ability to reject low-frequency noise (motion artefact), the proposed two-electrode circuit showed significantly more EMG noise in the output.

Using the data of one of the subjects, digital bandpass filtering (using MATLAB) was able to attenuate both low and high frequency noise such that a clearly identifiable heartbeat (R-wave) could be extracted both while standing (i.e. holding onto the rollator) as well as when walking (i.e. pushing the rollator). This is a very encouraging result and suggests that perhaps a larger study should be undertaken to quantify the robustness of heart rate measurement achievable with the Smart Rollator in a larger population.

For periods when shifting weight, changing grip and gripping strongly on the handles, however, noise levels visually overwhelmed the ECG signal. Occasionally clipping was also noticed. Although the post-processed signal (i.e. with digital filtering) did show clear R-waves, there were also some confounding "false" heartbeat signals observed (i.e. pulses similar in shape to an $\mathrm{R}$-wave). More sophisticated digital processing techniques may give more reliable results.

As there are significant variances in the quality of ECG in a population, the results from this pilot study of four subjects should be taken as preliminary. Based on this data, a larger study could be beneficial in further identifying the applicability of ECG monitoring in the Smart Rollator.

\subsubsection{Speed/Distance Monitoring}

Engineering specifications available for magnets and magnetic sensors (in particular, reed and Hall type sensors), unfortunately, are insufficient to adequately analyse the response of systems using these components. The ability to reliably detect a magnet passing near a 
sensor depends on both the nominal distance the sensor is mounted from the wheel and the amount of variance in these settings (due to wheel wobble, wheel play, magnet strength and mounting variances, etc.), in addition to numerous other difficult to quantify environmental factors. By characterizing the system with a chosen set of components (i.e. magnets and sensors), we were able to define sensor mounting distances (from the wheel) and sensor separations which resulted in a robust quadrature signal (i.e. no missing pulses or badly encoded movement).

The ability to predict valid quadrature encoding required that sensor "pulse width jitter" bounds be known and that the sensors be spaced such that pulses from the sensors overlap correctly even under conditions of worse variance. Correct overlap could be ascertained by inspection of the results from the proposed system's characterization tests.

\subsection{Future Work}

\subsubsection{ECG Acquisition}

$>$ A study focusing on the evaluation of alternative materials for the dry grip electrodes could help identify advantages and limitations with respect to electrical characteristics, usability (i.e. biocompatibility, comfort, maintenance/lifetime), and cost.

Confirming the preliminary results from the usage tests could be accomplished through a follow-up study with a larger number of healthy subjects. It could have as its objective to identify how the quality of the ECG signals (using both the proposed circuit and a conventional monitoring system - such as a Holter) varies across a population when performing controlled activities. In other words, to evaluate the 
mean and the variance of ECG signal quality, as a function of amplifier design, activity, and perhaps, age, sex, etc.

$>$ Further to this, performing "field" tests in which actual rollator users are monitored during normal daily activities. This could help in identifying the additional effects of ailment type and real-world usage scenarios on ECG quality and usability.

\subsubsection{Speed/Distance Monitoring}

For this study, to simplify the analysis, the effect of hysteresis (inherent in the reed switches) was not analysed nor factored into the design. A closer look at the benefits and limitations of this effect, how significant these may be, and how these further bound the set of stable configurations, could be useful (if for no other reason, to establish that hysteresis is not a significant effect!)

The presented system design process (in Chapter 4) could apply equally well to a design using Hall effect switches or devices. (A Hall effect switch is a Hall effect devices with integrated threshold detectors and a digital output). An analysis of the benefits (e.g. reliability) and the challenges (e.g. overcoming higher power consumption by cycling power), in the context of the presented process, would be beneficial and possibly offer an alternative to using reed switches (and possibly a better one). 


\section{Appendix A: Parts Specifications - ECG Amplifier}

\section{A.1 Wet Paste-On Electrode}

\begin{tabular}{|c|l|}
\hline Description & $\begin{array}{l}\text { NeuroPlus } 43 \text { is a small (2.5 cm square), aggressive } \\
\text { electrode designed for easy placement and superior } \\
\text { adhesion. Specially formulated adhesive solid gel } \\
\text { ensures full sensor contact and excellent readings. }\end{array}$ \\
\hline Image & \\
\hline $\begin{array}{c}\text { Size } \\
\text { (including backing) }\end{array}$ & $2.5 \mathrm{~cm}$ square \\
\hline Size & $1.12 \mathrm{~cm}$ Dia. (0.31 cm area) \\
\hline (electrode area) & $\begin{array}{l}\text { MERMED } \\
\text { Www.vermed.com }\end{array}$ \\
\hline Manuf Part No. & A10043 \\
\hline
\end{tabular}




\section{Termed}

\section{Introducing} Repositionable NeuroPlus

Vermed's new line of repostionable disposable electrodes for use m Sleep Sludies, EMG, NCS and EP.

- Neunothus 43 is a small (2.5 cm square), ageressive electrode desinned for easy placement and superor atheson. specially formulated adhesive sold wel ensures full sensor conact and excellent reathoss.

- NeuroPles 40,41 and 42 are designed wh a mone pentle athesive pel for use in senkitive areas Specallv formulated athestre gel fis alka repositiona ble to entuire proper pheement. Sutes range from $2.2 \mathrm{~cm}$ xquare $103.5 \mathrm{~cm}$ square.

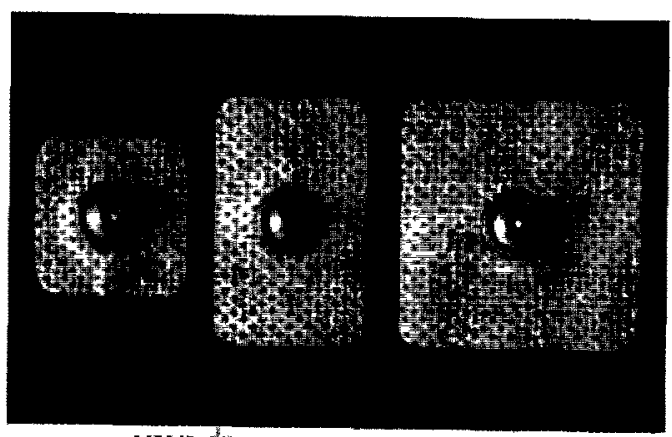

$t^{\frac{1}{y^{4}}}$

Heture:

solitiol

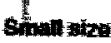

Benartit

Hepostifonable angeloun up

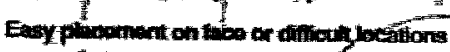

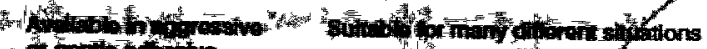

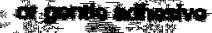

the now

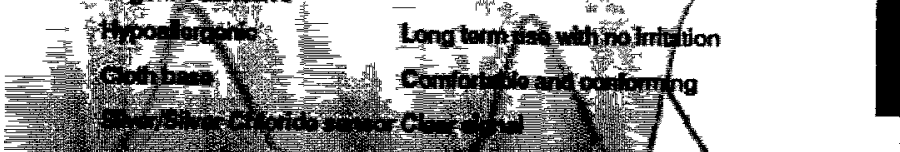

encingryt

int

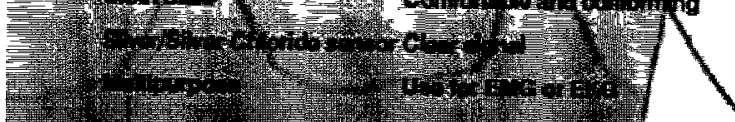

$+1 \times 2 \times 3$

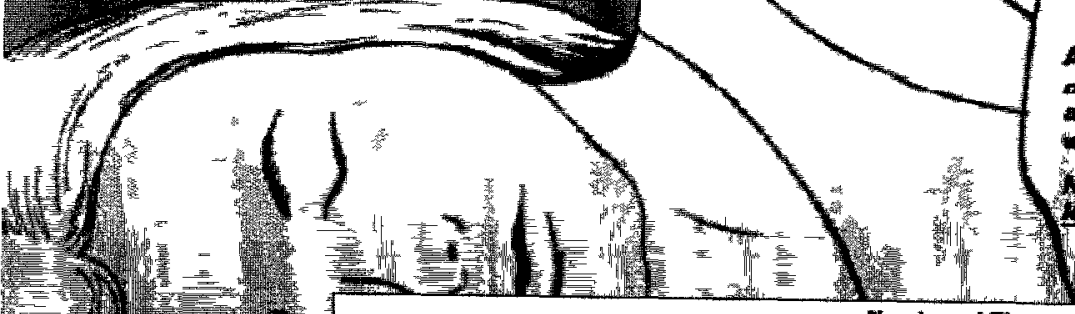

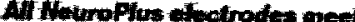

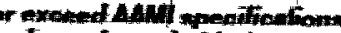

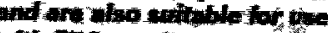
Hit Enomantar.

the

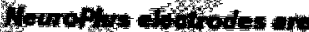

atortat

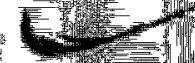

\begin{tabular}{|c|c|c|c|c|}
\hline \multirow[b]{2}{*}{ Pant Number } & \multirow[b]{2}{*}{ Doscripation } & \multicolumn{3}{|c|}{ Number of Eloctrodes } \\
\hline & & per strip & par pouch & por cass \\
\hline A10040-60 & $7 / 8^{*} \times 7^{\prime} 8^{*}$ Cloth & 3 & $\infty$ & 600 \\
\hline A $10041-60$ & 1-3/8 $\times 7 / 8^{-10}$ Cloth & 3 & $\infty$ & 600 \\
\hline A10042-60 & 1-3/ $\mathrm{B}^{-} \times 1-3 / \mathrm{B}^{-1}$ Cloth & 3 & - & 000 \\
\hline A10043 & $1 \times 10^{*}$ Cloth & $\mathbf{3}$ & 30 & 300 \\
\hline A1005060 & i" Danneter Foam Wet Gal & $\mathbf{3}$ & 60 & 600 \\
\hline
\end{tabular}

$000 \cdot 245,4025$

$602-453-5975$ + $6 x+902-453-93+6$

\section{VER $M E D$}

Figure 5-1: Datasheet for the A10043 Ag-AgCl paste-on electrode. 


\section{A.2 Instrumentation Amplifier}

\begin{tabular}{|c|c|}
\hline Description & $\begin{array}{l}\text { Single-Supply, Rail-to-Rail, Low Cost, } \\
\text { Low Power, Instrumentation Amplifier, } \\
\text { 8-DIP }\end{array}$ \\
\hline Manufacturer & $\begin{array}{l}\text { Analog Devices } \\
\text { www.analog.com }\end{array}$ \\
\hline Manuf Part No. & AD623ANZ \\
\hline Distributor & $\begin{array}{l}\text { Digi-Key Corporation } \\
\text { www.digikey.ca }\end{array}$ \\
\hline Part No. & AD623ANZ-ND \\
\hline $\begin{array}{c}\text { Price } \\
(2010.11 .24)\end{array}$ & $\begin{array}{l}\$ 4.44 \text { CAN, QTY } 1 \\
\$ 2.76 \text { CAN, QTY } 100\end{array}$ \\
\hline
\end{tabular}




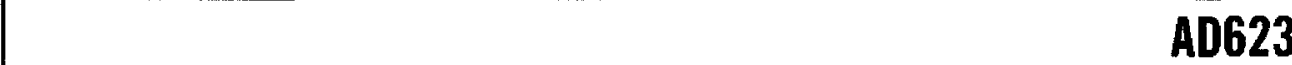

FEATURES

Easy to use

Higher performance than discrete design

Single-supply and dual-supply operation

Rail-to-rail output swing

Input voltage range extends 150 mV below

ground (single supply)

Low power, $550 \mathrm{\mu A}$ maximum supply current

Gain set with one external resistor

Gain range: 1 (no resistor) to 1000

High accuracy dc performance

$0.10 \%$ gain accuracy $(G=1)$

$0.35 \%$ gain accuracy $(G>1)$

10 ppm maximum gàn drift ( $G=1$ )

$200 \mu V$ maximum input offset voltage (AD623A)

$2 \mu \mathrm{V} /{ }^{\circ} \mathrm{C}$ maximum input offset drift (AD623A)

$100 \mu V$ maximum input offset voltage (AD623B)

$1 \mu \mathrm{V} / \mathrm{C}$ maximum input off set drift (AD623B)

25 nA maximum input bias current

Noise: $35 \mathrm{nV} / \sqrt{ } \mathbf{H z}$ RTI noise 1 kHz (G = 1 )

Excellent ac specifications

$90 \mathrm{~dB}$ minimum CMPR $(\mathrm{G}=10) ; 70 \mathrm{~dB}$ minimum CMRR $(G=1\}$

at $60 \mathrm{~Hz}, 1 \mathrm{k \Omega}$ source imbalance

$800 \mathrm{kHz}$ bandwidth $(\mathrm{G}=1)$

$20 \mathrm{ps}$ settling time to $0.01 \%(G=10)$

\section{APPLICATIONS}

Low power medical instrumentation

Transducer interfaces

Thermocouple amplifiers

Industrial process controls

Difference amplifiers

Low power data acquisition

\section{GENERAL DESCRIPTION}

The AD623 is an integrated single-supply instrumentation amplifier that delivers rail-to-rail output swing on a $3 \mathrm{~V}$ to $12 \mathrm{~V}$ supply. The AD623 offers supenor user flextiluty by allowing single gain set resstor programming and by conforming to the 8-lead industry standard pmout configuration. With no external resistor, the AD623 is configured for unity gam $\left(C_{T}=1\right)$, and with an external resistor, the AD623 can be programmed for gains up to 1000 .

\section{CONNECTION DIAGRAM}

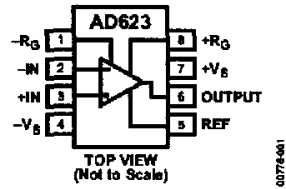

Figure I 8-Lead POIP (N), SOK (R), and MSOF (RM) Packages

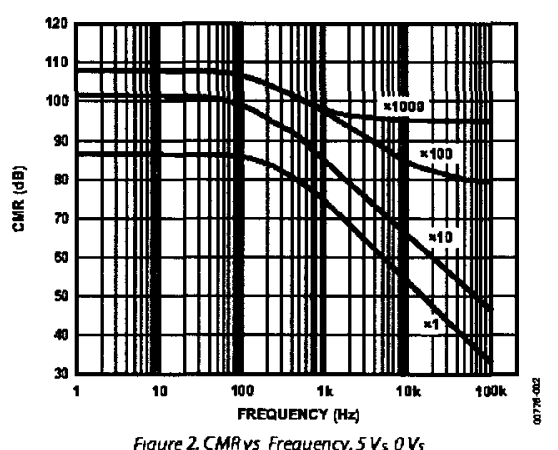

The AD623 holds errors to a munimum by providing superior ac CMRR that increases with increas ing gain Line notse, as well as line harmonics, are rejected because the CMRR remains constant up to $200 \mathrm{~Hz}$. The AD 623 has a wide input commonmode range and can amplify signals that have a common-mode voltage $150 \mathrm{mV}$ below ground. Although the design of the AD623 was optimized to operate from a single supply, the AD623 still provides superior performance when operated from a dual voltage supply $( \pm 2.5 \mathrm{~V}$ to $\pm 60 \mathrm{~V})$

Low power consumption ( $15 \mathrm{~mW}$ at $3 \mathrm{~V}$ ), wide supply voltage range, and rall to rall output swing make the AD623 1deal for battery-powered applications. The rail to raul output stage maxımuzes the dynamic range when operating from low supply voltages. The AD 623 replaces discrete instrumentation amplifier designs and offers supertor lineanty, temperature stablity, and reliability in a munimum of space

Figure 5-2: Datasheet for AD623 instrumentation amplifier. 


\section{A.3 Operational Amplifier}

\begin{tabular}{|c|c|}
\hline Description & $\begin{array}{l}\text { QUAD } 880-n A / C h \text { RAIL-TO-RAIL } \\
\text { INPUT/OUTPUT OPERATIONAL AMPLIFIER, } \\
5.5 \text { KHZ GBWP, 14SOIC }\end{array}$ \\
\hline Manufacturer & $\begin{array}{l}\text { Texas Instruments } \\
\text { www.ti.com }\end{array}$ \\
\hline Manuf Part No. & TLV2404IDR \\
\hline Distributor & $\begin{array}{l}\text { Digi-Key Corporation } \\
\text { www.digikey.ca }\end{array}$ \\
\hline Distr Part No. & 296-10537-1-ND \\
\hline $\begin{array}{c}\text { Price } \\
(2010.11 .24)\end{array}$ & $\begin{array}{l}\$ 4.72 \text { CAN, QTY } 1 \\
\$ 3.46 \text { CAN, QTY } 100\end{array}$ \\
\hline
\end{tabular}




\section{TLV2401, TLV2402, TLV2404 \\ FAMLY OF 880nA/Ch RAL-TO-RAIL INPUT/OUTPUT OPERATIONAL AMPLIFIERS WTH REVERSE BATTERY PROTECTION LLS244B - FEBRUARY 2000 - REVISED NOVEMBER 2000}

- Micro-Power Operation . . < $1 \mu$ AVChannel

- Input Common-Mode Range Exceeds the Ralls... - $0.1 \mathrm{~V}$ to $\mathrm{V}_{\mathrm{CC}}+5 \mathrm{~V}$

- Reverse Battery Protection Up To $18 \mathrm{~V}$

- Rall-to-Rall InputOutput

- Galn Bandwidth Product ... $5.5 \mathrm{kHz}$

- Supply Voltage Range ... $2.5 \mathrm{~V}$ to $16 \mathrm{~V}$

- Speciffed Temperature Range - $\mathbf{T}_{\mathrm{A}}=\mathbf{0}^{\circ} \mathrm{C}$ to $\mathbf{7 0}^{\circ} \mathrm{C} \ldots$. Commercial Grade - $T_{A}=-40^{\circ} \mathrm{C}$ to $125^{\circ} \mathrm{C}$... Industrial Grade

- Uitrasmall Packaging

- 5-Pin SOT-23 (TLV2401)

- BPin MSOP (TLV2402)

- Universal OpAmp EVM (Refer to the EVM Selection Gulde SLOU060)

description

The TLV240x family of single-supply operational amplifiers has the lowest supply current avalable today at only 880 nAper channet Reverse battery protection guards the amplfier from an overcurrent condition due to improper battery Installation For harsh envronments, the inputs can be taken $5 \mathrm{~V}$ above the postive supply rail without damage to the device

The low supply current is coupled with extremely low input bias currents enabling them to be used with mega- $\Omega$ resistors makıng them ideal for portable, long active life, applications DC accuracy is ensured with a low typical offset voltage as low as $390 \mu \mathrm{V}$ CMRR of $120 \mathrm{~dB}$ and minımum open loop gain of $130 \mathrm{~V} / \mathrm{mV}$ at $27 \mathrm{~V}$

The maximum recommended supply voltage is as high as $16 \mathrm{~V}$ and ensured operation down to $25 \mathrm{~V}$, with electrical charactenstıcs spectied at $27 \mathrm{~V}, 5 \mathrm{~V}$ and $15 \mathrm{~V}$ The $25-\mathrm{V}$ operation makes it compatible with $4-10 n$ battery-powered systems and many micro-power microcontrollers avallable today including Tl's MSP430

All members are avalable In PDIP and SOIC wth the singles in the small SOT-23 package duals in the MSOP, and quads in TSSOP

SELECTION OF SINGLE SUPPLY OPERATIONAL AMPLIFIER PRODUCTST

\begin{tabular}{|c|c|c|c|c|c|c|}
\hline DEVICE & $\begin{array}{l}v_{C C} \\
(V)\end{array}$ & $\begin{array}{l}V_{10} \\
(\mathrm{mV})\end{array}$ & $\begin{array}{c}\text { BW } \\
(\mathrm{MHz})\end{array}$ & $\begin{array}{c}\text { SLEW RATE } \\
\left(V^{\prime} / \mu\right)\end{array}$ & $\begin{array}{l}\text { Iccleh } \\
(\mu \mathrm{A})\end{array}$ & RALL TO-RAIL. \\
\hline TLV240x‡ & $25-16$ & 0390 & 0005 & 0002 & 0880 & $1 / 0$ \\
\hline TLV224x & $25-12$ & 0600 & 0005 & 0002 & 1 & 10 \\
\hline TLV2211 & $27-10$ & 0450 & 0065 & 0025 & 13 & 0 \\
\hline TLV/245x & $27-6$ & 0020 & 022 & 0110 & 23 & $1 / 0$ \\
\hline TLV225x & $27-8$ & 0200 & 02 & 012 & 35 & 0 \\
\hline
\end{tabular}

$\dagger$ All specfications are typical values measured at $5 \mathrm{~V}$

FThis device aiso offers $18 \mathrm{~V}$ reverse battery profection and $5 \mathrm{~V}$ over the rall operation on the unputs
Operational Amplifier

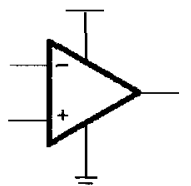

SUPPLY CURRENT

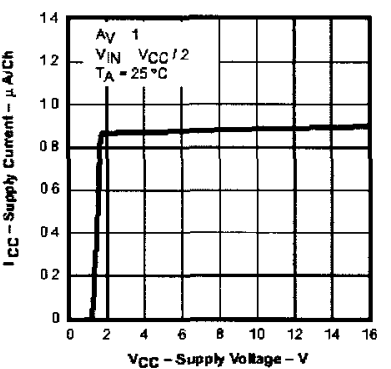

$V_{C C}-$ Supply Vallage $-V$

Figure 5-3: Datasheet for Texas Instruments TLV2404 operational amplifier. 


\section{A.4 Lithium Coin Battery}

\begin{tabular}{|c|c|}
\hline Description & $\begin{array}{l}\text { Lithium Coin Battery, 3V, } 20 \mathrm{~mm} \times 3.2 \\
\mathrm{~mm}, 225 \mathrm{mAh}, 0.1 \mathrm{oz}(2.9 \mathrm{~g})\end{array}$ \\
\hline Manufacturer & $\begin{array}{l}\text { Panasonic Batteries } \\
\text { www.panasonic.com }\end{array}$ \\
\hline Manuf Part No. & CR2032 \\
\hline Distributor & $\begin{array}{l}\text { Digi-Key Corporation } \\
\text { www.digikey.ca }\end{array}$ \\
\hline Distr Part No. & P189-ND \\
\hline $\begin{array}{c}\text { Price } \\
(2010.11 .24)\end{array}$ & $\begin{array}{l}\$ 0.31 \text { CAN, QTY } 1 \\
\$ 0.22 \text { CAN, QTY } 100\end{array}$ \\
\hline
\end{tabular}


the swiss power source

renata batteries

\section{CR2032}

3V Lithium Battery

Swiss Made

\section{Technical Data Sheet}

\section{Specifications}

Chemical System

Nominal Voltage

Rated Capacity

Standard Discharge Current

Max. Cont. Discharge Current

Average Weight

Temperature Range

Self Discharge at $23^{\circ} \mathrm{C}$
$\mathrm{Li} / \mathrm{MnO}_{2}$

$3 \mathrm{~V}$

$235 \mathrm{mAh}$

$0,4 \mathrm{~mA}$

$3,0 \mathrm{~mA}$

$2,8 \mathrm{~g}$

$-30-+70^{\circ} \mathrm{C}$

$<1 \%$ / year

\section{Dimensions}

(According to IEC 60086)

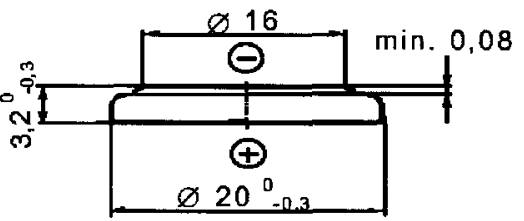

\section{Performance}
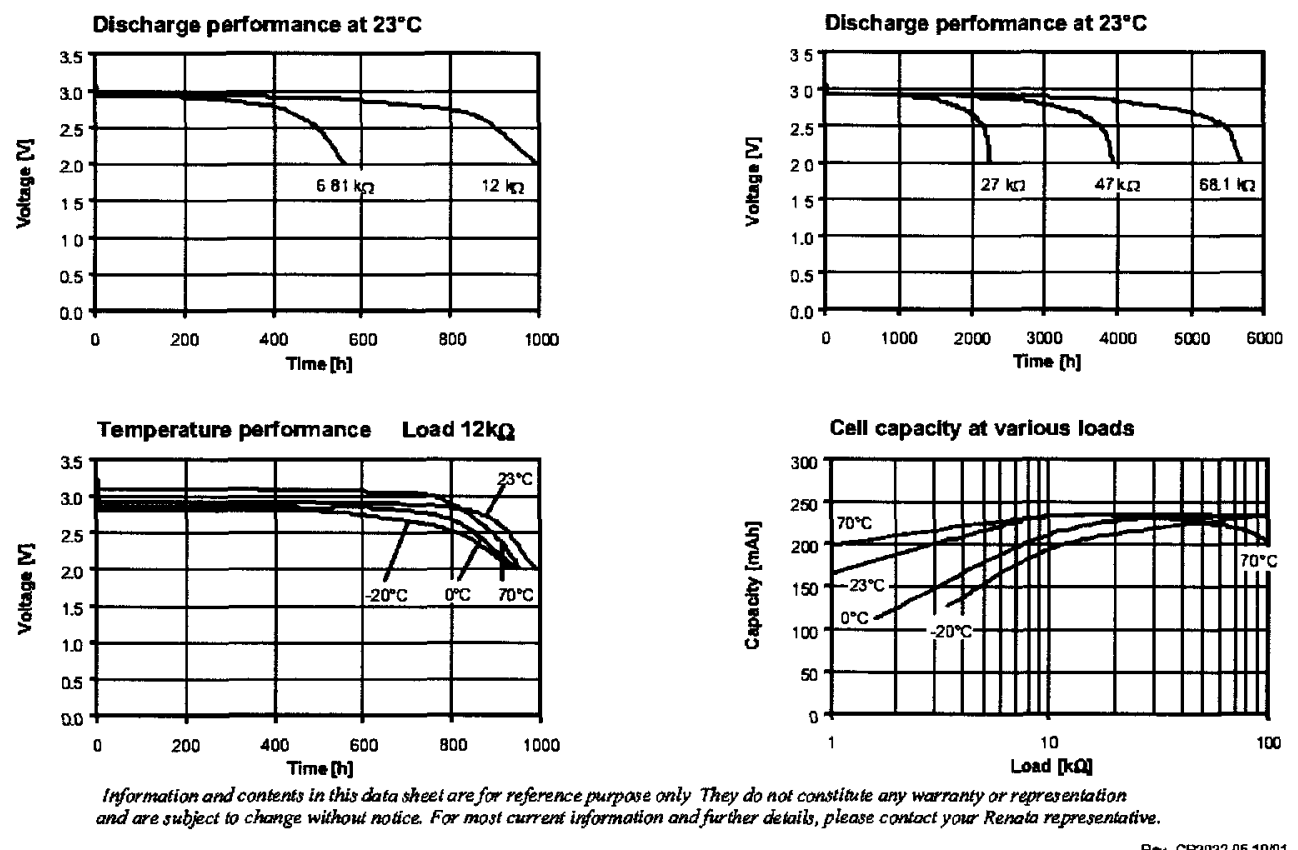

Information and contents in this data sheet are for reference puppase only they do thot constitute any warronty or repres entation and are subject to change without notice. For most current information and further details, please cantoct your Renata representative.

Rer CPR032 0510001

Figure 5-4: Datasheet for Renata CR2032 lithium coin battery. 


\section{Appendix B: Parts Specifications - Speed/Distance}

\section{A.5 Magnets}

\begin{tabular}{|c|c|c|}
\hline & 4 & 3 \\
\hline Material & \multicolumn{2}{|c|}{ Neodymium Iron Boron (Nd-Fe-B) alloy } \\
\hline $\begin{array}{c}\text { Magnetic } \\
\text { Energy Product }\end{array}$ & \multicolumn{2}{|c|}{$\begin{array}{l}18-45 \mathrm{MGOe} \text { (megagauss-oersteds) } \\
143-358 \mathrm{KJ} / \mathrm{m}^{3}\end{array}$} \\
\hline Description & \multicolumn{2}{|c|}{$\begin{array}{l}\text { Rare earth magnets made from Neodymium Iron } \\
\text { Boron and nickel plated. } \\
\text { (Source: distributor's catalogue) }\end{array}$} \\
\hline Manufacturer & \multicolumn{2}{|l|}{$\begin{array}{l}\text { Radial Magnet Inc. } \\
\text { www.radialmagnet.com }\end{array}$} \\
\hline Distributor & \multicolumn{2}{|l|}{$\begin{array}{l}\text { Digi-Key Corporation } \\
\text { www.digikey.ca }\end{array}$} \\
\hline Dimensions & $\begin{array}{l}0.250^{\prime \prime} \text { Dia } \times 0.250^{\prime \prime ~ H} \\
(6.35 \mathrm{~mm} \times 6.35 \mathrm{~mm})\end{array}$ & $\begin{array}{l}0.500^{\prime \prime} \text { Dia } \times 0.125 " \mathrm{H} \\
(12.70 \mathrm{~mm} \times 3.18 \mathrm{~mm})\end{array}$ \\
\hline Part No. & 469-1003-ND & 469-1002-ND \\
\hline $\begin{array}{c}\text { Price } \\
(2010.11 .24)\end{array}$ & $\begin{array}{l}\$ 0.43 \text { CAN, QTY } 1 \\
\$ 0.36 \text { CAN, QTY } 100\end{array}$ & $\begin{array}{l}\$ 0.72 \text { CAN, QTY } 1 \\
\$ 0.56 \text { CAN, QTY } 100\end{array}$ \\
\hline
\end{tabular}




\section{A.6 Reed Switch}

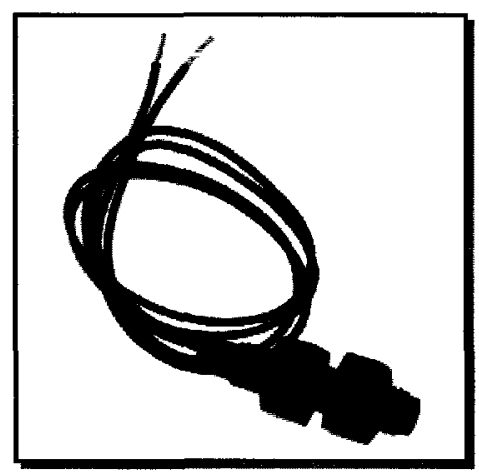

\begin{tabular}{|c|c|}
\hline Description & $\begin{array}{l}\text { Small plastic barrel sensor with a } 5 / 16^{\prime \prime} \\
\times 24 \text { thread, } 38.1 \mathrm{~mm}\left(1.500^{\prime \prime}\right) \text { long } \\
\text { with normally open contacts. }\end{array}$ \\
\hline Manufacturer & $\begin{array}{l}\text { Hamlin Electronics } \\
\text { www.hamlin.com }\end{array}$ \\
\hline Distributor & $\begin{array}{l}\text { Digi-Key Corporation } \\
\text { www.digikey.ca }\end{array}$ \\
\hline Part No. & 59065-010-ND \\
\hline $\begin{array}{c}\text { Price } \\
(2010.11 .24)\end{array}$ & $\begin{array}{l}\$ 4.04 \text { CAN, QTY } 1 \\
\$ 2.33 \text { CAN, QTY } 100\end{array}$ \\
\hline
\end{tabular}


HAMLIN

www.hamlin.com

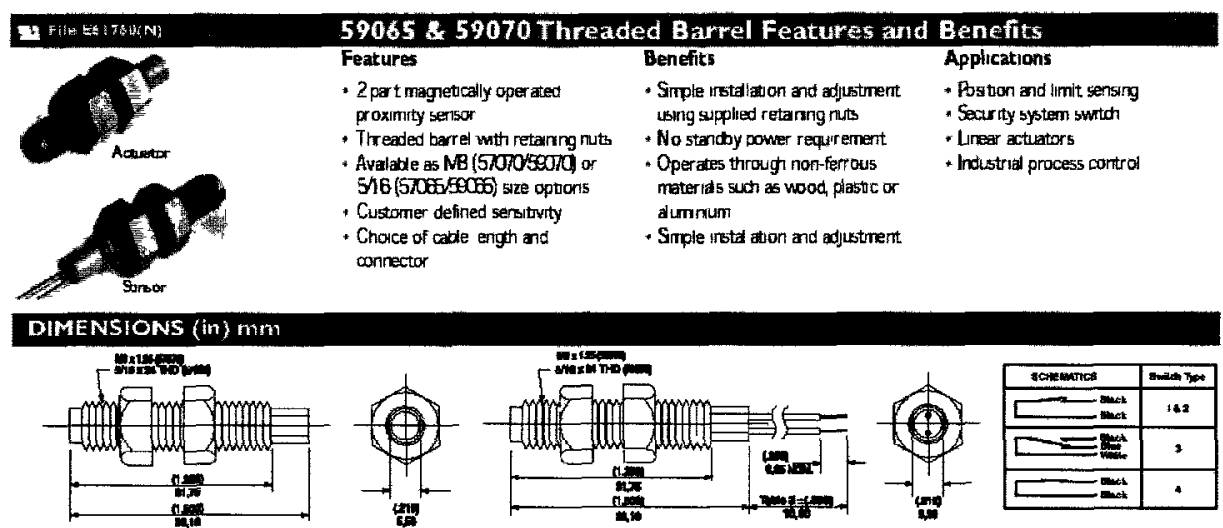

\section{CUSTOMER OPTIONS - Switching Specifications}

\begin{tabular}{|c|c|c|c|c|c|c|}
\hline \multicolumn{3}{|l|}{$\begin{array}{l}\text { TABLE ! } \\
\text { Contact Type }\end{array}$} & $\begin{array}{c}\text { Normally } \\
\text { Open }\end{array}$ & $\begin{array}{c}\text { Normally Open } \\
\text { High Voltage }\end{array}$ & $\begin{array}{c}\text { Change } \\
\text { Oreer }\end{array}$ & $\begin{array}{c}\text { Narmally } \\
\text { Closed }\end{array}$ \\
\hline \multicolumn{3}{|l|}{ Soutech Type } & 1 & 2 & 3 & 4 \\
\hline & Power & Wart max & 10 & 10 & 5 & 5 \\
\hline \multirow[t]{2}{*}{ Voluagc } & Switeching & Vde $\max$ & 200 & 300 & 775 & $7 \pi$ \\
\hline & Broskdown & Voce nun & 200 & 450 & 200 & 200 \\
\hline \multirow[t]{2}{*}{ Cuicert } & Sontetang & $A \max$ & 05 & 05 & DEs & DES \\
\hline & GaTy & A $\max$ & 12 & 15 & 75 & 15 \\
\hline \multirow[t]{2}{*}{ Ressyances } & Lantext Intial & $\Omega \max$ & 02 & 02 & 02 & 02 \\
\hline & Irsulatanton & $\Omega$ กา & 70 & 70 & $70^{\prime}$ & $70^{\prime}$ \\
\hline Lapestance & Comaxa & $p=p p$ & 03 & O2 & 03 & 03 \\
\hline \multirow[t]{2}{*}{ Tenperacure } & Bperaing & 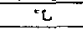 & $-00+15$ & $20 \mathrm{~m}+15$ & $-00 x+155$ & $400+108$ \\
\hline & storag & $t$ & $-65 t 0+1 C E$ & $650+100$ & $60+100$ & $650+106$ \\
\hline \multirow[t]{2}{*}{ Tinnc } & Operter & ins & 70 & 10 & 30 & 30 \\
\hline & Rectesse & $\mathrm{ms} \quad \mathrm{ng}$ & 10 & 10 & 30 & 30 \\
\hline Ylack & $11 \mathrm{~ms} 1 / 2 \mathrm{sin}$ & $c$ max & 100 & 100 & 50 & 50 \\
\hline Vilus soon & $50 \approx 0 \mathrm{~Hz}$ & $\mathrm{c}$ max. & 30 & 30 & 30 & 30 \\
\hline
\end{tabular}

CUSTOMER OPTIONS - Sensitivity, Cable Length and Termination Specification TABLL 2

Sensibunty Optonse

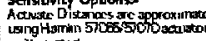
sollusuated
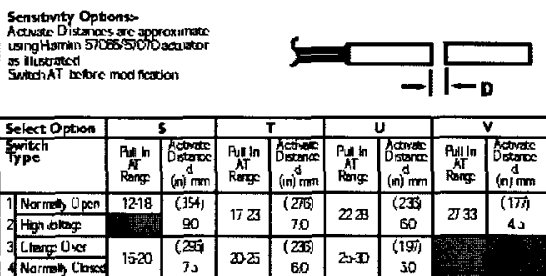

\begin{tabular}{|c|c|}
\hline \multicolumn{2}{|c|}{ 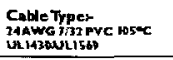 } \\
\hline \multicolumn{2}{|c|}{ Senderd Longtrs } \\
\hline $\begin{array}{l}\text { SEECT } \\
\text { OPTION }\end{array}$ & $\begin{array}{l}\text { EABLE } \\
\text { LENGTH } \\
\text { (III) } \mathrm{mm}\end{array}$ \\
\hline 여 & (394) 100 \\
\hline $\mathbf{q}$ & (1า 19300 \\
\hline $\mathbb{C B}$ & $019 \times 9500$ \\
\hline of & 12953750 \\
\hline$\infty 6$ & $(3937) 1000$ \\
\hline
\end{tabular}

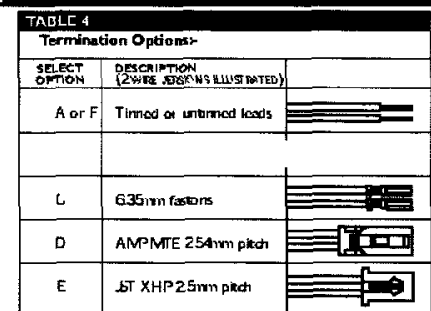

\section{ORDERING INFORMATION}

NB 57065/57070 actuator sold separately

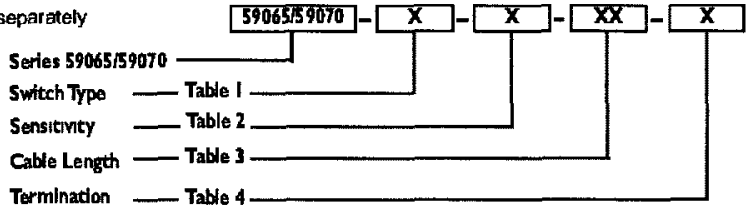

Termination Table 4

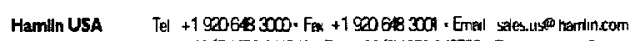

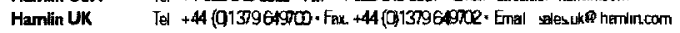

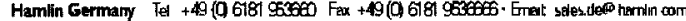

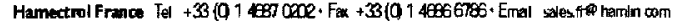

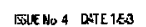

Figure 5-5: Hamlin 59065 reed switch datasheet. 


\section{References}

Agnello, D., \& Earl, B. (2008). Smart Rollator Usage Monitoring System. Undergraduate Project Report, Carleton University, Department of Systems and Computer Engineering, Faculty of Engineering, Ottawa.

Allegro Microsystems Inc. (2010). A1205 Continuous-Time Bipolar Switch. Retrieved Nov 28, 2010, from Allegro Microsystems web site:

http://www.allegromicro.com/en/Products/Part_Numbers/1205/1205.pdf

Allegro Microsystems Inc. (2010). A1391 Hall Effect Sensor IC Datasheet. Retrieved Nov 28, 2010, from Allegro Microsystems web site:

http://www.allegromicro.com/en/Products/Part_Numbers/1391/1391.pdf

Asahi Kasei EMD Corporation. (2009). EQ-711L Linear Hall Effect IC Data Sheet. Retrieved Nov 28, 2010, from GMW Associates web site:

http://www.gmw.com/magnetic_sensors/asahi/documents/eq-7111_gmw.pdf

B.C. Ministry of Health Planning. (2004). Prevention of falls and injuries among the elderly.

Bailey, J. J., Berson, A. S., Garson Jr, A., Horan, L. G., Macfarlane, P. W., Mortara, D. W., et al. (1990). Recommendations for standardization and specifications in automated

electrocardiography: bandwidth and digital signal processing. Circulation , 81 (2), 730-739.

Baker, B. C. (2003). Select the Right Operational Amplifier for your Filtering Circuits - Analog Design Note ADN003. Microchip Technology Inc.

Burke, M. J. (1994). Low-power ECG amplifier/detector for dry-electrode heart rate monitoring. Medical and Biological Engineering and Computing , 32 (6), 678-683.

Burke, M. J., \& Assambo, C. (2007). An improved micro-power pre-amplifier for dry-electrode ECG recording. Proceedings of the 11th WSEAS International Conference on Circuits. 11, pp. 234-239. World Scientific and Engineering Academy and Society.

Burke, M. J., \& Gleeson, D. T. (2000). A micropower dry-electrode ECG preamplifier. IEEE Transactions on Biomedical Engineering , 47 (2), 155-162.

Caggiano, D., \& Reisman, S. (2002). Respiration derived from the electrocardiogram: a quantitative comparison of three different methods. Proceedings of the 1996 IEEE TwentySecond Annual Northeast Bioengineering Conference (pp. 103-104). IEEE.

Chan, A. D., \& Green, J. R. (2008). Smart Rollator Prototype. Proceeding of the 2008 IEEE International Workshop on Medical Measurements and Applications (MeMeA) (pp. 97-100). Ottawa, ON: IEEE. 
Chimene, M. F., \& Pallas-Areny, R. (2000). A comprehensive model for power line interference in biopotential measurements. IEEE Transactions on Instrumentation and Measurement , 49 (3), 535-540.

Cooper, R. A., Thorman, T., Cooper, R., Dvorznak, M. J., Fitzgerald, S. G., Ammer, W., et al. (2002). Driving characteristics of electric-powered wheelchair users. Archives of physical medicine and rehabilitation, 83 (2), 250-255.

Dawson, W. W., \& Doddington, H. W. (1973). Phase distortion of biological signals: extraction of signal from noise without phase error. Electroencephalography and Clinical Neurophysiology, $34(2), 207-211$.

Dobrev, D. P., Neycheva, T., \& Mudrov, N. (2008). Bootstrapped two-electrode biosignal amplifier. Medical and Biological Engineering and Computing , 46 (6), 613-619.

Dobrev, D. (2004). Two-electrode low supply voltage electrocardiogram signal amplifier. Medical and Biological Engineering and Computing , 42 (2), 272-276.

Dobrev, D., \& Daskalov, I. (2002). Two-electrode biopotential amplifier with current-driven inputs. Medical and Biological Engineering and Computing , 40 (1), 122-127.

Everett, H. R., Pastore, T. H., \& Bonner, K. (1999). Mobile Robots for Outdoor Security Applications.

Fuller, G. F. (2000). Falls in the elderly. American Family Physician, 61 (7), 2159.

Gillum, R. F., Makuc, D. M., \& Feldman, J. J. (1991). Pulse rate, coronary heart disease, and death: the NHANES I Epidemiologic Follow-up Study. American Heart Journal , 121 (1), 172177.

Gruetzmann, A., Hansen, S., \& Müller, J. (2007). Novel dry electrodes for ECG monitoring. Physiological Measurement , 28, 1375-1390.

Gupta, R., Goldstein, R., \& Brooks, D. (2006). The acute effects of a rollator in individuals with COPD. Journal of Cardiopulmonary Rehabilitation , 26 (2), 107-111.

Hamlin Inc. (2007). Application Note AN102A - Ampere*Turn Versus $m$ T and Gauss. Retrieved Nov 28, 2010, from Hamlin Products web site: http:/www.hamlin.com/specSheets/AN102AAmpere-turn_versus_mT_and_Gauss.pdf

Hamlin Inc. (2008). Application Note AN104 - Reed Switch and Reed Sensor Activation. Retrieved Nov 28, 2010, from Hamlin Products web site: http://www.hamlin.com/specSheets/AN104.pdf

Harper, C. M., \& Lyles, Y. M. (1988). Physiology and complications of bed rest. Journal of the American Geriatric Society , 36, 1047-1054.

Hirvensalo, M., Rantanen, T., \& Heikkinen, E. (2000). Mobility difficulties and physical activity as predictors of mortality and loss of independence in the community-living older population. Journal of the American Geriatric Society, 48, 493-498. 
Hoffmann, K. P., \& Ruff, R. (2007). Flexible dry surface-electrodes for ECG long-term monitoring. Proceeding of the 29th Annual International Conference of the IEEE Engineering in Medicine and Biology Society, 2007 (pp. 5739-5742). IEEE.

Honeywell International. (2005). SS49E Series Linear Position Sensor - Specs. Retrieved Nov 28, 2010, from Honeywell Sensing and Control web site:

http://sensing.honeywell.com/index.cfm?ci_id=140301\&la_id=1\&pr_id=128770

Huhta, J. C., \& Webster, J. G. (1973). 60-Hz interference in electrocardiography. IEEE Transactions on Biomedical Engineering , 20 (2), 91-101.

Kannel, W. B., Kannel, C., Paffenbarger Jr, R. S., \& Cupples, L. A. (1987). Heart rate and cardiovascular mortality: the Framingham Study. American Heart Journal , 113 (6), 1489-1494.

Kim, J., Hong, J., Kim, N., Cha, E., \& Lee, T. S. (2007). Two Algorithms for Detecting Respiratory Rate from ECG Signal. World Congress on Medical Physics and Biomedical Engineering 2006 (pp. 4069-4071). Springer.

Korhonen, I., Parkka, J., \& Van Gils, M. (2003). Health monitoring in the home of the future. 22 (3), 66-73.

Kutiyanawala, A., Kulyukin, V., \& LoPresti, E. (2006). A rollator-mounted wayfinding system for the elderly. Proceedings of the 8th international ACM SIGACCESS conference on computers and accessibility (pp. 245-246). ACM.

LaBarge, B. M., \& Gutierrez-Miravete, E. (2008). Finite Element Model of a Magnet Driven Reed Switch. Proceedings of the COMSOL Conference. Boston.

Lankenau, A., \& Röfer, T. (2000). Smart Wheelchairs - State of the Art in an Emerging Market. Zeitschrift Künstliche Intellgenz , 14 (4), 37-39.

Lu, G., Brittain, J. S., Holland, P., Yianni, J., Green, A. L., Stein, J. F., et al. (2009). Removing ECG noise from surface EMG signals using adaptive filtering. Neuroscience letters , 462 (1), 1419.

National Semiconductor. (1969, Mar). High Q Notch Filter. Retrieved Dec 10, 2010, from National Semiconductor Linear Briefs web site: http:/www.national.com/ms/LB/LB-5.pdf

Pallas-Areny, R. (1986). On the reduction of interference due to common mode voltage in twoelectrode biopotential amplifiers. IEEE transactions on bio-medical engineering , 33 (11), 10431046.

Pan, J., \& Tompkins, W. J. (1985). A real-time QRS detection algorithm. IEEE Transactions on Biomedical Engineering , 32 (3), 230-236.

Probst, V. S., Troosters, T., Coosemans, I., Spruit, M. A., Pitta, F. O., Decramer, M., et al. (2004). Mechanisms of Improvement in Exercise Capacity Using a Rollator in Patients With COPD. Chest , 126 (4), 1102-1107. 
Radial Magnet Inc. (2010). Neodymium/Samarium Magnets. Retrieved Nov 28, 2010, from Radial Magnet web site: http://radialmagnet.com/neodymium.html

Rangayyan, R. M. (2002). Biomedical signal analysis. IEEE.

Redfern, M. S., Hughes, R. E., \& Chaffin, D. B. (1993). High-pass filtering to remove electrocardiographic interference from torso EMG recordings. Clinical Biomechanics , 8 (1), 4448.

Renata SA. (2006, Dec). CR2032 3V Lithium Battery Technical Data Sheet. Retrieved Dec 10, 2010, from Renata Batteries web site: http://www.renata.com/pdf/3vlithium/CR2032_v06.pdf

Rentschler, A. J., Simpson, R., Cooper, R. A., \& Boninger, M. L. (2008). Clinical evaluation of Guido robotic walker. Journal of Rehabilitation Research and Development , 45 (9), 1281-1294.

Richard, E., \& Chan, A. D. (2010). Design of a gel-less two-electrode ECG monitor. Proceeding of the 2010 IEEE International Workshop on Medical Measurements and Applications (MeMeA) (pp. 92-96). Ottawa, ON: IEEE.

RLS Merilna Tehnika. (2009, June 19). LM13 Magnetic Ring Encoder System Data Sheet. Retrieved Nov 28, 2010, from RLS Rotary and Linear Motion Sensors web site: www.rls.si/document/LM13D01.pdf

ROHM Semiconductor. (2010). BU52742GUL Bipolar Latch Hall IC Data Sheet. Retrieved Nov 28, 2010, from ROHM Semiconductor web site: http://www.rohm.com/products/databook/sensor/pdf/bu52742gul-e.pdf

Romero, D. H., \& Stelmach, G. E. (2003). Changes in postural control with aging and Parkinson's disease. IEEE Engineering in Medicine and Biology Magazine , 22 (3), 27-31.

Ruha, A., Sallinen, S., \& Nissila, S. (1997). A real-time microprocessor QRS detector system with a 1-ms timing accuracy for the measurement of ambulatory HRV. IEEE Transactions on Biomedical Engineering , 44 (3), 159-167.

Searle, A., \& Kirkup, L. (2000). A direct comparison of wet, dry and insulating bioelectric recording electrodes. Physiological Measurement, 21 (2), 271-283.

Sonenblum, S. E., Sprigle, S., Harris, F. H., \& Maurer, C. L. (2008). Characterization of power wheelchair use in the home and community. Archives of physical medicine and rehabilitation, 89 (3), 486-491.

Spinelli, E. M., Pallàs-Areny, R., \& Mayosky, M. A. (2003). AC-coupled front-end for biopotential measurements. IEEE Transactions on Biomedical Engineering , 50 (3), 391-395.

Tayler, D., \& Vincent, R. (1983). Signal distortion in the electrocardiogram due to inadequate phase response. IEEE Transactions on Biomedical Engineering , 30 (6), 352-356.

Thakor, N. V., \& Webster, J. G. (1980). Ground-free ECG recording with two electrodes. IEEE Transactions on Biomedical Engineering (12), 699-704. 
Thakor, N. V., Webster, J. G., \& Tompkins, W. J. (2007). Estimation of QRS complex power spectra for design of a QRS filter. IEEE Transactions on Biomedical Engineering , 31 (11), 702706.

Tolerico, M. L., Ding, D., Cooper, R. A., Spaeth, D. M., Fitzgerald, S. G., Cooper, R., et al. (2007). Assessing mobility characteristics and activity levels of manual wheelchair users. Journal of Rehabilitation Research and Development , 44 (4), 561.

Tung, J., Gage, W., Zabjek, K., Brooks, D., Maki, B., Mihailidis, A., et al. (2007). iWalker: A 'real-world' mobility assessment tool. Proceedings of the 30th Canadian Medical \& Biological Engineering Conference. Toronto ON: CMBES.

Turcotte, M., \& Schellenberg, G. (2007). A Portrait of Seniors in Canada, 2006. Statistics Canada.

van Boxtel, A. (2001). Optimal signal bandwidth for the recording of surface EMG activity of facial, jaw, oral, and neck muscles. Psychophysiology, 38 (1), 22-34.

Warlar, R., \& Eswaran, C. (1991). Integer coefficient bandpass filter for the simultaneous removal of baseline wander, 50 and $100 \mathrm{~Hz}$ interference from the ECG. Medical and Biological Engineering and Computing , 29 (3), 333-336.

Wasson, G., Sheth, P., Alwan, M., Granata, K., Ledoux, A., \& Huang, C. (2004). User intent in a shared control framework for pedestrian mobility aids. Proceedings of the 2003 IEEE/RSJ International Conference on Intelligent Robots and Systems. 3, pp. 2962-2967. IEEE.

Webster, J. G. (2009). Medical instrumentation: application and design. Wiley-India.

Winter, B. B., \& Webster, J. G. (1983). Driven-right-leg circuit design. IEEE Transactions on Biomedical Engineering, 30 (1), 62-66.

Winter, B. B., \& Webster, J. G. (1983). Reduction of interference due to common mode voltage in biopotential amplifiers. IEEE Transactions on Biomedical Engin, 30 (1), 58-62.

Yoo, S. K., Kim, N. H., Song, J. S., Lee, T. H., \& Kim, K. M. (1997). Simple self-tuned notch filter in a bio-potential amplifier. Medical and Biological Engineering and Computing , 35 (2), 151-154.

Yu, H., Spenko, M., \& Dubowsky, S. (2003). An adaptive shared control system for an intelligent mobility aid for the elderly. Autonomous Robots , 15 (1), 53-66. 